\title{
Effects of Radial Hydrides on PWR Cladding Ductility following Drying and Storage
}

\section{Spent Fuel and Waste Disposition}

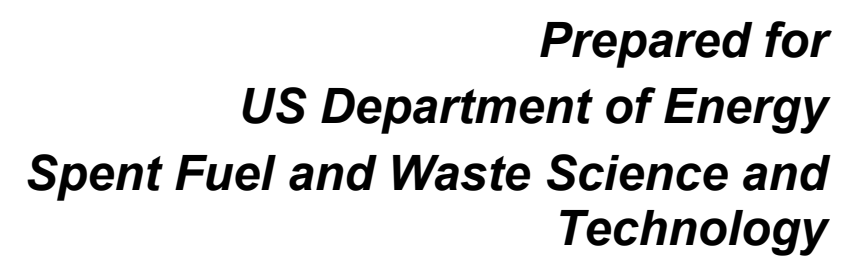

Argonne National Laboratory M.C. Billone, T.A. Burtseva, J.M. Garcia-Infanta (ENUSA) 



\section{DISCLAIMER}

This information was prepared as an account of work sponsored by an agency of the U.S. Government. Neither the U.S. Government nor any agency thereof, nor any of their employees, makes any warranty, expressed or implied, or assumes any legal liability or responsibility for the accuracy, completeness, or usefulness, of any information, apparatus, product, or process disclosed, or represents that its use would not infringe privately owned rights. References herein to any specific commercial product, process, or service by trade name, trade mark, manufacturer, or otherwise, does not necessarily constitute or imply its endorsement, recommendation, or favoring by the U.S. Government or any agency thereof. The views and opinions of authors expressed herein do not necessarily state or reflect those of the U.S. Government or any agency thereof. 



\section{SUMMARY}

The purpose of this research effort is to determine the effects of canister and/or cask drying, transfer and storage on radial hydride precipitation in, and potential embrittlement of, high-burnup (HBU) pressurized water reactor (PWR) cladding alloys during cooling for a range of peak drying-storage temperature (PCT) values and hoop stresses. Extensive radial-hydride precipitation could lower failure hoop stresses and strains, relative to limits established for as-irradiated cladding from discharged fuel rods, at temperatures below the ductility transition temperature (DTT), which has been referred to as the ductile-to-brittle transition temperature (DBTT) in previous reports. During post-storage transport, hoop stresses will be induced by "pinch-type" loading at grid spacers. Such loading is considered to be mild for normal conditions of transport and more severe for hypothetical accident conditions.

Ring compression tests (RCTs), which simulate pinch-type loading, are used as screening tests to measure cladding ductility as a function of RCT temperature from which the DTT is determined. Tests have been conducted with pressurized and sealed cladding rodlets heated to $350^{\circ} \mathrm{C}$ and $400^{\circ} \mathrm{C}$ followed by slow cooling $\left(5^{\circ} \mathrm{C} / \mathrm{h}\right)$. After simulated drying and storage, the DTT was determined as a function of the PCT and the peak hoop stress for HBU PWR cladding alloys. Previous results indicated that the DTT for $\mathrm{HBU} Z \mathrm{ZIRLO}{ }^{\mathrm{TM}}$ is a strong function of cladding hoop stress in the narrow range of $90 \pm 3 \mathrm{M} \mathrm{Pa}$. However, the higher hydrogen content of the 93-94 M Pa hoop-stress samples may have contributed to the higher DTT $\left(125 \pm 5^{\circ} \mathrm{C}\right)$ as compared to the lower DTT $\left(<30^{\circ} \mathrm{C}\right)$ for $87-89 \mathrm{MPa}$ samples with lower hydrogen contents. HBU M $5 \circledR$ results indicated that its DTT is a strong function of hydrogen content and a weaker function of peak hoop stress within the range tested (89-142 M Pa).

Prior to conducting RCTs with HBU cladding rings, twenty RCT benchmark tests were conducted with asfabricated $17 \times 17 \mathrm{M} 5{ }^{\circledR}$ cladding of two wall thicknesses: $0.61 \mathrm{~mm}$ and $0.57 \mathrm{~mm}$. Displacement rates were $0.05 \mathrm{~mm} / \mathrm{s}$ and $5 \mathrm{~mm} / \mathrm{s}$ (reference), test temperatures were $25^{\circ} \mathrm{C}$ and $120^{\circ} \mathrm{C}$, and displacements were in the range of $0.7 \mathrm{~mm}$ to $1.7 \mathrm{~mm}$ (reference). Some tests were conducted with the "cold-lab" Instron 5566 screw-type machine, whose calibration-verification was performed within the past year. The remaining tests were conducted using the in-glove-box Instron 8511 servo-hydraulic machine, which has been used to test all irradiated cladding rings. The benchmark results served many useful purposes: (a) calibrationverification of the Instron 8511 load cell, (b) demonstration that Instron 8511 results have not changed during the past 6 years, (c) generation of additional data to allow an ASTM standard to be written for RCTs, and (d) determination of an empirical relationship between maximum load $\left(P_{\max }\right)$ and ring length $(L)$, wall thickness $\left(h_{m}\right)$ and mid-wall radius $\left(R_{\text {mid }}\right)$. This relationship is significant because it can be used to normalize $P_{\max }$ results for as-fabricated and irradiated samples, to assess hardening due to irradiation, and to determine the extent of possible annealing during the heat-treatment phase of the tests.

For the M5 ${ }^{\circledR}\left(652 F 2\right.$ with 80 wppm hydrogen) and ZIRLO ${ }^{\mathrm{TM}}$ (646D with 390 wppm hydrogen) rodlets tested previously, the degree of axial continuity of radial hydrides was assessed through examination of metallographic images of multiple cross sections of RCT samples. The radial hydride continuity factor (RHCF) was determined for about 40 overlapping images around each cross section. Significant variations were observed in the cross-section averaged RHCF and the length and location of the longest radial hydride for each cross section. For samples that exhibited cracking, crack length and circumferential location at the ring mid-span and at one end of the ring were also used to assess the degree of axial continuity. For the M $5{ }^{\circledR}$ rodlet cross sections, the average RHCF varied from $30 \%$ to $54 \%$ of the cladding wall thickness, the peak RHCF varied from $63 \%$ to $100 \%$, and the circumferential location of the longest radial hydride was different for each of the six cross sections within an axial span of 15 $\mathrm{mm}$. The average RHCF for all readings was $44 \pm 18 \%$. These results suggest relatively poor axial continuity of radial hydrides in 80 -wppm $\mathrm{HBU} M 5{ }^{\circledR}$ following cooling from $350^{\circ} \mathrm{C}$ and $89-\mathrm{MPa}$ hoop 
stress. However, the brittle sample tested at $25^{\circ} \mathrm{C}$ had mid-span wall cracks of $100 \%$ (at $120^{\prime}$ clock) and $80 \%$ (at 6 o'clock). These cracks were primarily radial, appeared to follow a continuous radial hydride path, and were observed visually (at 1X) at both ends of the intact compressed ring. Although the radial hydrides in HBU M 5 ®clearly lacked axial continuity, individual hydrides appear to be close enough in the axial direction to promote cracking along the length of the compressed ring at room temperature.

For the 390 -wppm ZIRLO ${ }^{\mathrm{TM}}$ rodlet subjected to peak conditions of $350^{\circ} \mathrm{C}$ and $87-\mathrm{M} \mathrm{Pa}$ hoop stress, radial hydrides were relatively short and cracking, when it did occur, initiated at the outer surface of the hydride rim and propagated radially through the rim of circumferential hydrides, circumferentially along individual hydrides, and at $45^{\circ}$ through ductile metal between circumferential hydrides. The short radial hydrides appeared to have promoted some of the crack propagation. For the ring compressed at $60^{\circ} \mathrm{C}$, dominant cracks were observed at $30^{\prime}$ clock for the mid-span and end surfaces. The cracks followed zigzag paths, which were different at the mid-span and end cross sections. In terms of RHCF, the average RHCF varied from $10 \%$ to $17 \%$ within an axial span of $13 \mathrm{~mm}$, the maximum value varied from $26 \%$ to $52 \%$, and the circumferential orientation of the longest radial hydride varied from sample to sample. The average RHCF was $15 \pm 9 \%$. Although radial hydrides appeared to be discontinuous, cracking was controlled mostly by circumferential hydrides, which have a high degree of axial continuity.

The 390-wppm ZIRLO ${ }^{\mathrm{TM}}$ rodlet (646D) tested previously was subjected to peak conditions of $350^{\circ} \mathrm{C}$ and $87-\mathrm{M} \mathrm{Pa}$ for a $24-\mathrm{h}$ hold time followed by $5^{\circ} \mathrm{C} / \mathrm{h}$ cooling under decreasing pressure and hoop-stress. To better quantify the sensitivity of DTT to peak hoop stress, a new rodlet (646C) with the same oxide-layer and metal-wall thickness values and about the same hydrogen content was subjected to the same thermal history at a targeted peak hoop stress of $93 \mathrm{MPa}$. If previous conclusions were correct, this higher-stress rodlet should have precipitated longer radial hydrides ( $>30 \%$ of the cladding wall) and exhibited a DTT in the range of $125 \pm 5^{\circ} \mathrm{C}$. However, as indicated by the absence of radial-hydride precipitation, the $646 \mathrm{C}$ rodlet did not maintain full pressure throughout the thermal history, especially during cooling. The peak hoop stress at $350^{\circ} \mathrm{C}$ was shown to be $>55 \mathrm{MPa}$ and in the range of $63 \pm 8 \mathrm{MPa}$. Although the test did not achieve its designated purpose, the RCT results for 646C samples proved useful in evaluating the effects of short radial hydrides in the 646D samples on cladding ductility and $P_{\text {max. }}$ Also, based on recent FRAPCON calculations, peak hoop stresses $\leq 53 \mathrm{MPa}$ are relevant for standard PWR fuel rods during drying, transfer and storage. Thus, for $646 \mathrm{C}$ at $>55 \mathrm{M} \mathrm{Pa}$ peak stress, it does bound the FRAPCON results for standard PWR fuel rods. The $350^{\circ} \mathrm{C} / 93-\mathrm{M} \mathrm{Pa}$ test will be repeated with rodlet $646 \mathrm{~B}$, which should be very similar in geometry and hydrogen content to rodlets $646 \mathrm{C}$ and $646 \mathrm{D}$. The FRAPCON calculations gave $81 \mathrm{MPa}$ as a bounding hoop stress for Integral Fuel Burnable Absorber (IFBA with B-10) PWR rods. The 93-M Pa test results will help determine the margin for IFBA PWR fuel rods between calculated peak hoop-stress limits and hoop stresses for which the DTT will be $\geq 120^{\circ} \mathrm{C}$.

For FY2018 testing, experimental PCT values will be based on calculated fleet-bounding values for conduction and conduction-convection casks, as well as the NRC-recommended limit of $400^{\circ} \mathrm{C}$. Peak internal pressures and corresponding peak hoop stresses will be based on end-of-life rod internal pressures to be measured at room temperature for more than 10 of the 25 sister rods, as well as the sparse database gathered for legacy PWR fuel rods. These internal pressures, which are expected to be $\leq 5 \mathrm{MPa}$, will result in more realistic experimental peak stresses. Prior to receiving sister-rod cladding, Argonne will conduct tests with available high-hydrogen-content ( $>600-w p p m) ~ Z R L O{ }^{T M}$ cladding rodlets at relevant temperatures and pressures to determine the effects of thick hydride rims on ductility. These test results will be very useful in refining test matrices for sister-rod cladding. 


\section{CONTENTS}

SUM MARY. iii

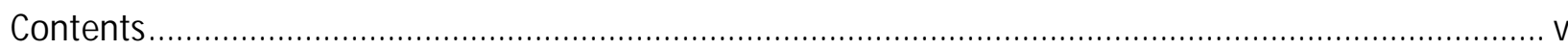

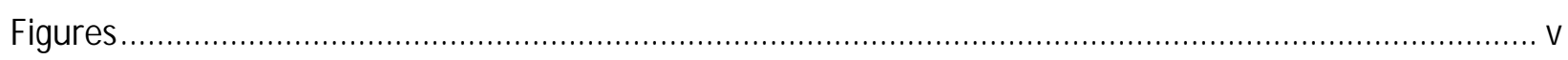

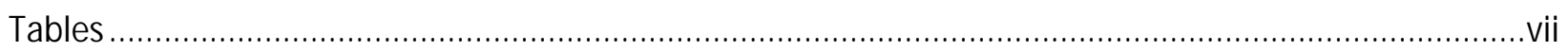

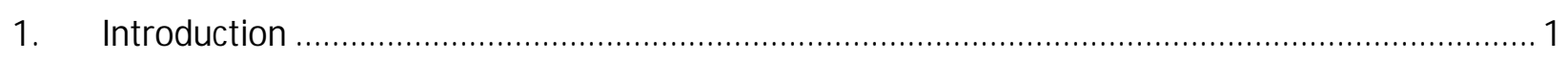

2. HBU Cladding M aterials and Test Methods ........................................................................... 5

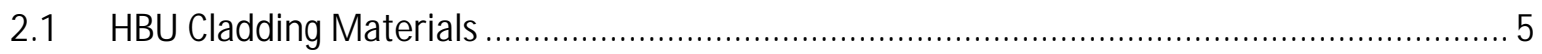

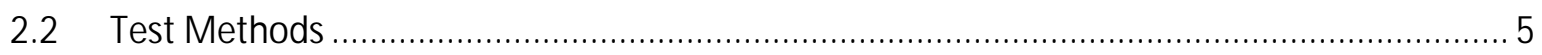

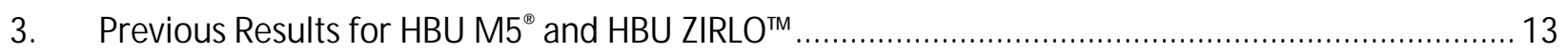

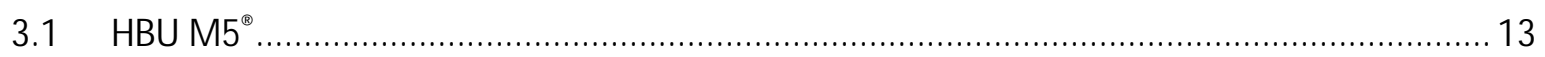

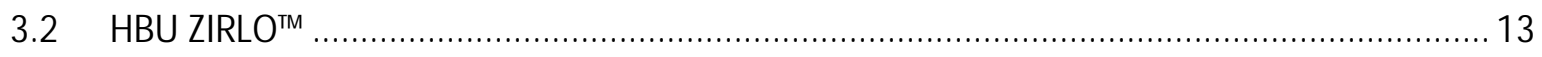

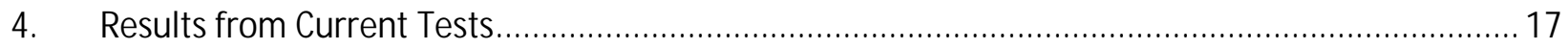

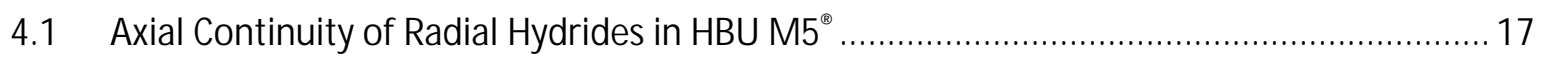

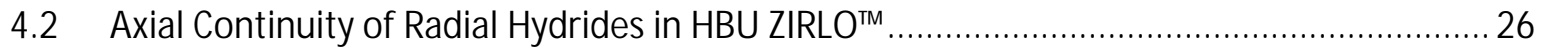

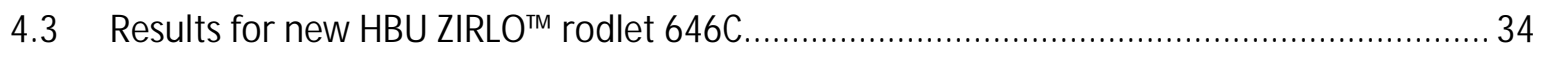

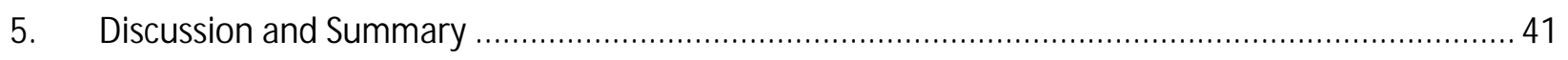

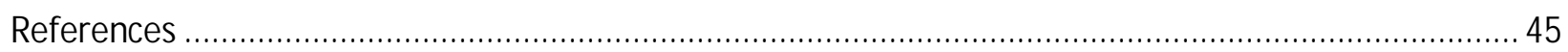

\section{FIGURES}

1 Steady-state curves for hydrogen dissolution and precipitation in Zr alloys................................ 3

2 Rodlet components: bottom end fixture, zirconia pellet, cladding segment, and top end fixture. ... 6

3 RCT measured load (P) and controlled displacement $(\delta)$.................................................... 7

4 Load-displacement curve for as-fabricated (AF) $M 5^{\circledR}$ ring tested at $\mathrm{RT}$ and $0.05 \mathrm{~mm} / \mathrm{s}$ to

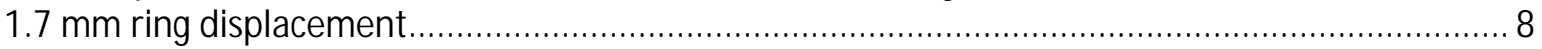

5 RCT benchmark results for determining the ratio of unloading/loading stiffness as a function of the traditional offset strain.

6 RCT ductility curves for $\mathrm{HBU} M 5^{\circledR}$ in the as-irradiated condition and following RHT at $350^{\circ} \mathrm{C}$ and $400^{\circ} \mathrm{CPCT}$.

7 DTT for HBU M $5{ }^{\circledR}$ Vs. hydrogen content following RHT at $350^{\circ} \mathrm{C}$ and $400^{\circ} \mathrm{C} \mathrm{PCT}$....................... 14

8 Summary of ductility data for $\mathrm{HBU} Z \mathrm{ZRLO}{ }^{\mathrm{TM}}$ following RHT at $400^{\circ} \mathrm{C}$ and $350^{\circ} \mathrm{C} \mathrm{PCTs}$.................. 15

9 DTT vs. hoop stress for HBU ZIRLO ${ }^{\mathrm{TM}}$ following RHT at $400^{\circ} \mathrm{C}$ and $350^{\circ} \mathrm{C} \mathrm{PCTs...........................} 15$

10 Sectioning diagram for HBU M $5^{\circledR}$ rodlet $652 \mathrm{~F} 2$ following RHT and RCT ..................................... 17

11 Longest radial hydride observed at the 12:30 orientation for the 652F2F cross section at the reference $(0 \mathrm{~mm})$ axial location: (a) 100X and (b) 200X.

12 Longest radial hydride (bottom of figure) observed at 3:30 for the 652F2F cross section at the 0.5-mm axial location: (a) 100X and (b) 200X 
13 Longest radial hydride observed at the $12: 30$ orientation for the $652 \mathrm{~F} 2 \mathrm{~F}$ cross section at the

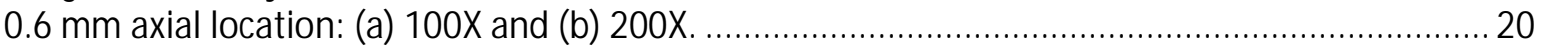

14 Longest radial hydride observed at the $5: 45$ orientation for the $652 \mathrm{~F} 2 \mathrm{G} 1$ cross section at the

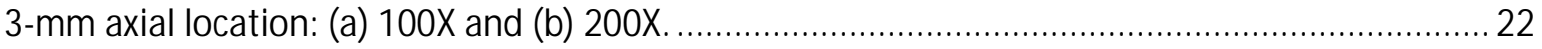

15 Longest radial hydride observed at the $11: 15$ orientation for the $652 \mathrm{~F} 2 \mathrm{G} 2$ cross section at the

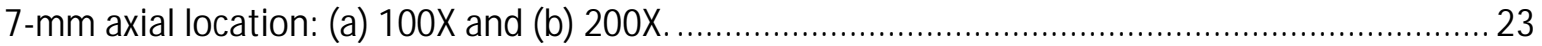

16 Long cracks observed at the mid-span cross section of compressed ring $652 \mathrm{~F} 2 \mathrm{H}$ at the $15-\mathrm{mm}$ axial location: (a) 12:00 o'clock and (b) 6:00 o'clock. ...................................................................... 24

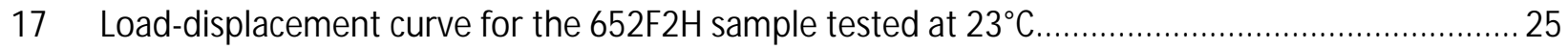

18 Sectioning diagram for the HBU ZIRLO ${ }^{\text {TM }}$ rodlet 646D following RHT and RCT .............................. 26

19 Images of the longest radial hydride observed for the $646 \mathrm{D} 6$ cross section $(0 \mathrm{~mm})$, which occurred

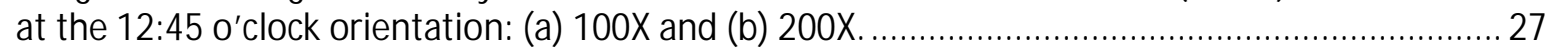

20 Major crack observed at the 3:00 o'clock orientation of the 646D4A cross section at the $-6-\mathrm{mm}$

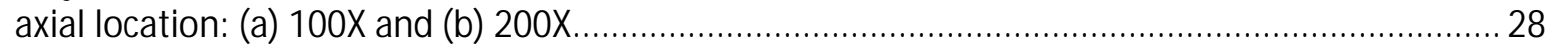

21 Image of the longest radial hydride for the 646D4A cross section $(-6 \mathrm{~mm})$, which occurred at the

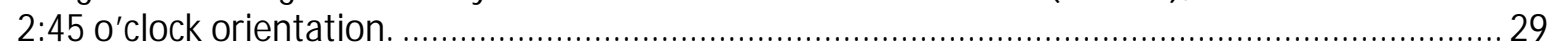

22 Major crack observed at the 3:00 o'clock orientation of the 646D4B cross section at the $-6-\mathrm{mm}$ axial location: (a) 100X and (b) 200X.................................................................................. 30

23 Images of the longest radial hydride for the 646D4B cross section $(-2 \mathrm{~mm})$, which occurred at the 8:45 o'clock orientation: (a) 100X and (B) 200X.................................................................. 31

24: Images of the longest radial hydride for the 646D7A cross section $(3 \mathrm{~mm})$, which occurred at the 8:45 o'clock orientation: (a) 100X and (B) 200X. 32

25 Images of the longest radial hydride for the 646D7B cross section $(7 \mathrm{~mm})$, which occurred at the 2:00 o'clock orientation: (a) 100X and (B) 200X ................................................................. 33

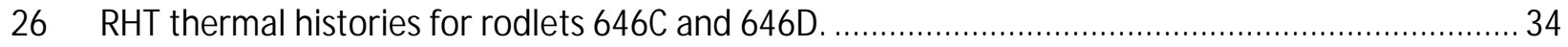

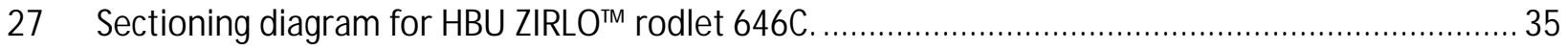

28 M icrograph from a 100X image taken at the 12:00 o'clock orientation of the $646 \mathrm{C} 6$ cross section.36

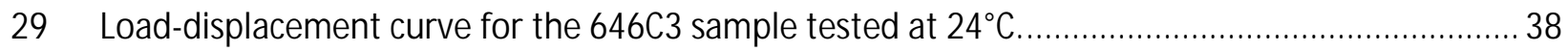

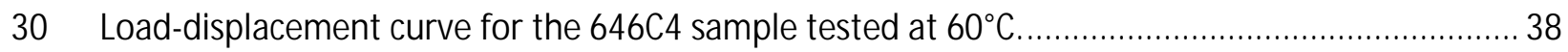

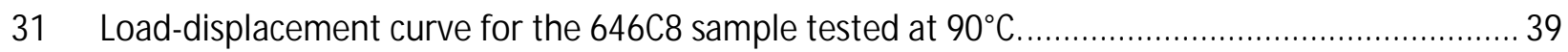

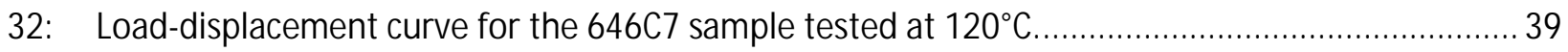




\section{TABLES}

1 Summary of $\mathrm{HBU} \mathrm{M} 5^{\circledR}$ and ZIRLO ${ }^{\mathrm{TM}}$ cladding materials used in studies of cladding ductility following simulated drying and storage RHT at PCT.

2 RCT benchmark results for $17 \times 17 \mathrm{AF} \mathrm{M} 5^{\circledR}$ cladding with wall thicknesses of $0.61 \mathrm{~mm}$ and $0.57 \mathrm{~mm}$. The maximum load in the last column is normalized by $(8.0-\mathrm{mm} / \mathrm{L})\left(0.0689 /\left[\mathrm{h}_{\mathrm{m}} / \mathrm{R}_{\mathrm{mid}}\right]\right)^{2.44} \ldots \ldots \ldots \ldots 10$

3 Results of metallographic examination for six cross sections of HBU M 5 @rodlet $652 F 2$.............. 25

4 Results of metallographic examination for five cross sections of HBU ZIRLO ${ }^{\text {TM }}$ rodlet 646D.......... 34

5 RCT results for HBU ZIRLO ${ }^{\mathrm{TM}}$ rodlets $646 \mathrm{C}(0 \%$ RHCF) and 646D (15 $\pm 9 \%$ RHCF)............................. 40 


\section{ACRONYMS, UNITS AND SYMBOLS}

\section{ACRONYMS}

$\begin{array}{ll}\text { AF } & \text { as-fabricated } \\ \text { ANL } & \text { Argonne National Laboratory } \\ \text { CWSRA } & \text { cold-worked, stress-relief annealed } \\ \text { DBTT } & \text { ductile-to-brittle transition temperature } \\ \text { DOE } & \text { U.S. Department of Energy } \\ \text { DTT } & \text { ductility transition temperature } \\ \text { EOL } & \text { end of life } \\ \text { EPRI } & \text { Electric Power Research Institute } \\ \text { FEA } & \text { finite element analysis } \\ \text { HBU } & \text { high burnup } \\ \text { IFBA } & \text { Integral Fuel Burnable Absorber } \\ \text { ISG } & \text { Interim Staff Guidance } \\ \text { NRC } & \text { Nuclear Regulatory Commission } \\ \text { PCT } & \text { peak cladding temperature } \\ \text { PWR } & \text { pressurized water reactor } \\ \text { RCT } & \text { ring compression test } \\ \text { RHCF } & \text { radial hydride continuity factor (in \%) } \\ \text { RHT } & \text { radial-hydride treatment } \\ \text { RIP } & \text { rod internal pressure } \\ \text { RT } & \text { room temperature } \\ \text { RXA } & \text { recrystallized-annealed } \\ \text { TM T } & \text { thermal-mechanical treatment } \\ \text { WBT } & \text { wide beam theory } \\ \text { Zry-2 } & \text { Zircaloy-2 } \\ \text { Zry-4 } & \text { Zircaloy-4 } \\ & \end{array}$

\section{UNITS}

$\begin{array}{ll}{ }^{\circ} \mathrm{C} & \text { degree Celsius } \\ \mathrm{GWd} / \mathrm{MTU} & \text { giga-watt-days per metric tonne of uranium } \\ \mathrm{h} & \text { hour } \\ \mathrm{K} & \text { degree Kelvin } \\ \mathrm{kN} & \text { kilo-newton } \\ \mathrm{m} & \text { meter } \\ \mathrm{mm} & \text { millimeter } \\ \mu \mathrm{m} & \text { micro meter (micron) } \\ \mathrm{MPa} & \text { mega-pascal } \\ \mathrm{N} & \text { newton } \\ \mathrm{S} & \text { second } \\ \text { wppm } & \text { weight parts per million }\end{array}$




\section{SYMBOLS}

\begin{tabular}{|c|c|}
\hline $\mathrm{C}_{\mathrm{H}}$ & total hydrogen content in weight parts per million (wppm) \\
\hline $\mathrm{C}_{\mathrm{HD}}$ & dissolved hydrogen content at temperature $T_{D}$ during heating (wppm) \\
\hline $\mathrm{CH}_{\mathrm{HP}}$ & $\begin{array}{l}\text { dissolved hydrogen content (wppm) needed to initiate hydride precipitation } \\
\text { during cooling at temperature } T_{p}\end{array}$ \\
\hline $\mathrm{D}_{\mathrm{mi}}$ & inner diameter of cladding alloy $(\mathrm{mm})$ \\
\hline $\mathrm{D}_{\mathrm{mo}}$ & outer diameter of cladding alloy (mm) \\
\hline$D_{0}$ & cladding outer diameter (includes outer-surface oxide layer if present, mm) \\
\hline$d_{p}$ & $\begin{array}{l}\text { permanent displacement (pre-test minus post-test diameter in loading } \\
\text { direction, } \mathrm{mm} \text { ) }\end{array}$ \\
\hline$d_{p} / D_{m o}$ & permanent strain (\%) \\
\hline$\delta$ & controlled sample displacement (mm) at the 12 o'clock sample position \\
\hline$\delta_{e}$ & elastic displacement (mm) \\
\hline$\delta_{\max }$ & maximum sample displacement $(\mathrm{mm})$ at the 12 o'clock sample position \\
\hline$\Delta p$ & pressure difference across cladding wall $\left(p_{i}-p_{0}, M P a\right)$ \\
\hline$\delta_{p}$ & $\begin{array}{l}\text { offset displacement }(\mathrm{mm}) \text { at the } 120^{\prime} \text { clock position relative to the static support } \\
\text { at the } 6 \mathrm{o}^{\prime} \text { clock }\end{array}$ \\
\hline$\delta_{\mathrm{p}} / \mathrm{D}_{\mathrm{mo}}$ & offset strain $(\%)$ \\
\hline$\Delta \mathrm{T}$ & temperature drop $\left({ }^{\circ} \mathrm{C}\right)$ per drying cycle \\
\hline$\Delta \mathrm{T}_{\mathrm{PD}}$ & $\begin{array}{l}\text { difference between hydride precipitation }\left(T_{P}\right) \text { and dissolution }\left(T_{D}\right) \text { temperatures } \\
\left({ }^{\circ} \mathrm{C}\right)\end{array}$ \\
\hline E & Young's modulus (GPa) \\
\hline$\left(\varepsilon_{\theta}\right)_{\max }$ & maximum hoop strain \\
\hline$h_{m}$ & cladding alloy wall thickness (mm) \\
\hline$h_{0 x}$ & thickness of outer surface oxide layer ( $\mu \mathrm{m})$ \\
\hline$K_{L C}$ & calculated loading slope (i.e., loading stiffness) for RCT samples (kN/mm) \\
\hline$K_{L M}$ & measured linearized loading slope (kN/mm) \\
\hline $\mathrm{Ku}_{u}$ & calculated linearized unloading slope (kN/mm) \\
\hline Kum & measured linearized unloading slope ( $\mathrm{kN} / \mathrm{mm})$ \\
\hline L & length of RCT sample (mm) \\
\hline$M_{\max }$ & maximum $\mathrm{RCT}$ bending moment $(\mathrm{N} \bullet \mathrm{m})$ \\
\hline $\mathrm{N}$ & Poisson's ratio \\
\hline $\mathrm{P}$ & measured RCT load at the 12 o'clock sample position (N) \\
\hline$P_{e}$ & load during elastic displacement $(\mathrm{N})$ \\
\hline $\mathrm{p}_{\mathrm{i}}$ & internal gas pressure (M Pa) \\
\hline$P_{\max }$ & maximum RCT load (N) \\
\hline$p_{0}$ & external gas pressure \\
\hline $\mathrm{R}_{\mathrm{mi}}$ & inner radius of cladding alloy (mm) \\
\hline$R_{\text {mid }}$ & mid-wall radius of cladding alloy $(\mathrm{mm})$ \\
\hline$\sigma_{\theta}$ & hoop stress (M Pa) \\
\hline $\mathrm{T}$ & temperature $\left({ }^{\circ} \mathrm{C}\right)$ \\
\hline $\mathrm{T}_{\mathrm{D}}$ & hydrogen dissolution temperature $\left({ }^{\circ} \mathrm{C}\right)$ \\
\hline$T_{p}$ & hydrogen precipitation temperature $\left({ }^{\circ} \mathrm{C}\right)$ \\
\hline
\end{tabular}




\section{Page intentionally blank}




\section{INTRODUCTION}

Structural analyses of high-burnup (HBU) fuel rods require cladding mechanical properties and failure limits to assess fuel behavior during long-term dry-cask storage, post-storage retrieval and transportation, and post-transport retrieval. License applications for transport casks containing HBU fuel assemblies with Zircaloy-2 (Zry-2), Zircaloy-4 (Zry-4) and ZIRLO ${ }^{\circledR}$ cladding have used properties and failure limits for as-irradiated cladding [1]. M5® cladding properties are not currently available. However, pre-storage drying-transfer operations and early stage storage subject cladding to higher tensile hoop stresses induced by higher temperatures and pressures relative to in-reactor operation and pool storage. Under these conditions, radial hydrides may precipitate during slow cooling and may introduce an embrittlement mechanism if the cladding temperature decreases below a critical point, which is defined in this work as the ductility transition temperature (DTT) and which was called the ductile-to-brittle transition temperature (DBTT) in previous reports. DTT was introduced to avoid potential confusion among materials experts who associate DBTT with body-centered-cubic metals. If embrittlement is predicted to occur, then failure hoop stresses and strains would have to be revised to account for this effect.

In Interim Staff Guidance-11, Revision 3 (ISG-11, Rev. 3), the Nuclear Regulatory Commission (NRC) recommends a peak cladding temperature (PCT) limit of $400^{\circ} \mathrm{C}$ for all fuel burnups under normal conditions of storage and short-term loading operations (e.g., drying, backfilling with inert gas, and transferring the canister or cask to the storage pad) [2]. During loading operations, repeated thermal cycling (repeated heat-up/cool-down cycles) may occur but should be limited to fewer than 10 cycles, with cladding temperature variations $(\Delta \mathrm{T})$ that are less than $65^{\circ} \mathrm{C}$ per cycle, according to ISG-11, Rev. 3 (see Fig. 1 at the end of this section for justification of $\Delta T<65^{\circ} \mathrm{C}$ per cycle). One concern for high-burnup (HBU) fuel cladding is the possible precipitation of radial hydrides, which could embrittle cladding in response to tensile hoop stresses caused by internal pressure loading and "pinch-type" loading during transport. Limits established in ISG-11, Rev. 3, relied on data available before 2002, which were primarily for low-burnup and non-irradiated/pre-hydrided Zry-4. At the time ISG-11, Rev. 3, was issued (2003), NRC recognized that data for HBU fuel cladding alloys were needed to determine the extent of radial-hydride-induced embrittlement under conditions relevant to drying-transfer operations and storage.

Argonne National Laboratory (ANL) has developed a test protocol for studying HBU cladding embrittlement that has been used to generate data for NRC. Experimentally, the protocol involves two steps: (a) radial-hydride treatment (RHT), during which HBU cladding is exposed to simulated dryingstorage temperature and hoop stress conditions, including slow cooling with decreasing stress, followed by (b) ring compression testing, in which rings sectioned from RHT HBU cladding are compressed to determine strength and ductility as a function of test temperature. The ring compression test (RCT) is used as a ductility screening test, and the RCT loading simulates the pinch-type loading on HBU cladding that occurs during normal cask transport and possible drop accidents. The protocol was used to generate DBTT data for HBU ZIRLO ${ }^{\mathrm{TM}}$ and Zry-4 $[3,4]$ (both efforts sponsored by NRC) and HBU M $5{ }^{\circledR}$ [sponsored by the U.S. Department of Energy (DOE)] [5]. Under DOE-sponsorship, ANL has also generated baseline characterization data and data for the strength and ductility of as-irradiated Zry-4, $\mathrm{ZRLO}{ }^{\mathrm{T}}$, and $\mathrm{M} 5{ }^{\circledR}$. These data are important not only for determining the potentially degrading effects of drying and early stage storage, but also for serving as reference properties for future evaluations of the effects of drying storage on these cladding alloys [6-8]. Reference 9 includes ANL data generated through September 30, 2013, including additional DOE-sponsored test results for HBU ZIRLO ${ }^{\mathrm{TM}}$ and M $5{ }^{\circledR}$ following cooling from $400^{\circ} \mathrm{C}$ and lower hoop stress levels ( 80 to $90 \mathrm{M} \mathrm{Pa}$ ). Reference 10 contains refined interpretations of previously generated data, as well as test results for HBU ZIRLO ${ }^{\mathrm{TM}}$ subjected to 3-cycle 
drying at $350^{\circ} \mathrm{C} \mathrm{PCT}$ and $93-\mathrm{MPa}$ peak hoop stress. Reference 11 contains additional data for asirradiated Zry-4 and for HBU ZIRLO ${ }^{\mathrm{TM}}$ following 1-cycle drying at $350^{\circ} \mathrm{C} \mathrm{PCT}$ and $94-\mathrm{M} \mathrm{Pa}$ peak hoop stress. Reference 12 presents results generated for $\mathrm{HBU}_{\mathrm{ZIRLO}}^{\mathrm{TM}}$ and $\mathrm{M} 5{ }^{\circledR}$ following $\mathrm{RHT}$ at $350^{\circ} \mathrm{C}$ and peak stresses in the range of 87-89 $\mathrm{MPa}$.

ANL test results indicate that susceptibility to radial-hydride precipitation during cooling is dependent on cladding alloy, thermal-mechanical treatment (TMT), total hydrogen content $\left(\mathrm{C}_{H}\right), \mathrm{C}_{\mathrm{H}}$ below the hydride rim, and peak RHT temperature and hoop stress. The combination of recrystallized-annealed (RXA) microstructure and low $C_{H}$ results in higher susceptibility of M5® to precipitation of long radial hydrides during cooling. For cold-worked, stress-relief-annealed (CWSRA) alloys, ZIRLO ${ }^{\mathrm{TM}}$ was found to be more susceptible to radial-hydride precipitation than Zry-4. The differences in the distribution of hydrides across the cladding wall (lower for ZIRLO ${ }^{\mathrm{TM}}$ below the hydride rim) may be partly responsible for this behavior [4].

Section 2 of this report describes the materials and test methods used in this program. It has been updated with additional RCT results for benchmark tests using as-fabricated $17 \times 17$ M 5®samples with wall thicknesses of $0.57 \mathrm{~mm}$ and $0.61 \mathrm{~mm}$ (reference value). Benchmark tests were conducted with both the Instron 5566 screw-type machine, whose calibration was recently verified, and the in-glove-box Instron 8511 servo-hydraulic machine, which has been used to test irradiated cladding rings. Benchmark test results are used to verify calibration of the Instron-8511, to generate additional data used to determine the decrease in unloading slope relative to the loading slope, and to determine the relationship between the maximum RCT load $\left(P_{\max }\right)$ and geometrical parameters (ring length, $L$, metal outer diameter, $D_{m o}$, and cladding wall thickness, $h_{m}$. Previous test results for HBU M 5 ßand HBU ZIRLO ${ }^{\text {TM }}$ are summarized in Section 3 for these materials following RHT at $400^{\circ} \mathrm{CPCT}$ and $350^{\circ} \mathrm{CPCT}$ for a range of hydrogen contents and peak hoop stresses [12].

New results for HBU M 5 @and HBU ZIRLO ${ }^{\mathrm{TM}}$ are presented in Section 4. This section contains assessments of radial-hydride continuity in the axial direction based on metallographic images for six cross sections within $15 \mathrm{~mm}$ for the M5 ${ }^{\circledR}$ rodlet (652F2) subjected to RHT at $350^{\circ} \mathrm{C} \mathrm{PCT} / 89-\mathrm{M}$ Pa peak hoop stress and for five cross sections within $13 \mathrm{~mm}$ for the ZIRLO ${ }^{\mathrm{TM}}$ rodlet (646D) subjected to RHT at $350^{\circ} \mathrm{C} \mathrm{PCT/87-}$ MPa peak hoop stress. Axial continuity is assessed based on crack length, location and morphology and on average and peak values for the radial hydride continuity factor (RHCF), which is used as the metric to determine the effective radial length of continuous radial-circumferential hydrides. For HBU ZIRLO ${ }^{\mathrm{TM}}$, a new test was conducted to determine the sensitivity of this alloy to peak hoop stress in the narrow range of $93 \pm 3 \mathrm{MPa}$. The ZIRLO ${ }^{\mathrm{TM}}$ rodlet (646D) tested in FY2016 exhibited relatively short radial hydrides and $\angle 30^{\circ} \mathrm{C}$ DTT following RHT at peak RHT conditions of $350^{\circ} \mathrm{C} / 87-\mathrm{MPa}$ for 24-hours and cooling at $5^{\circ} \mathrm{C} / \mathrm{h}$. The new ZIRLO ${ }^{\mathrm{TM}}$ rodlet $(646 \mathrm{C})$ used a segment below and adjacent to $646 \mathrm{D}$ in the parent fuel rod, which was projected to have about the same outer-diameter, oxide-layer thickness, metal wall thickness, and hydrogen content. It was subjected to the same heating-cooling cycle as the 646D rodlet. However, the room-temperature pressure was increased to achieve a peak hoop stress of 93-94 M Pa. Expectations were that radial hydrides would be longer ( $>30 \%$ of the cladding wall) and the DTT would be higher $\left(125 \pm 5^{\circ} \mathrm{C}\right)$.

To appreciate the influence of PCT (i.e., decrease from $400^{\circ} \mathrm{C}$ to $350^{\circ} \mathrm{C}$ ), stress, temperature cycling, and hydrogen content on radial hydride precipitation and embrittlement, it is important to review literature results for hydrogen dissolution and hydride precipitation. Unlike most, if not all, studies of radial hydride precipitation and subsequent ductility, the ANL RHT process includes decreasing internal gas pressure and hoop stress with decreasing temperature during cooling, as would occur in fuel rods during storage. Most researchers have used an actively pressurized cladding tube for which the pressure is kept 
constant during cooling. As such, it is important to understand the dependence of hydrogen solubility $\left(\mathrm{C}_{\mathrm{HD}}\right)$ on temperature $\left(\mathrm{T}_{\mathrm{D}}\right)$ during the heating phase and the content of dissolved hydrogen $\left(\mathrm{C}_{\mathrm{HP}}\right)$ needed to initiate precipitation at the precipitation temperature $\left(T_{p}\right)$ during the cooling phase. Figure 1 summarizes the data of Kearns [13], Kammenzind et al. [14], and McMinn et al. [15] for these parameters, as well as the temperature gap $\left(\Delta \mathrm{T}_{\mathrm{PD}}\right)$ between precipitation and dissolution. The results in Fig. 1 are used in Sections 3 and 4 to determine the cladding temperature and stress at which precipitation, especially radial hydride precipitation, can initiate. In particular, the solubility of hydrogen at $400^{\circ} \mathrm{C}$ is $206 \pm 5 \mathrm{wppm}$ and the precipitation temperature is $335^{\circ} \mathrm{C}$. The hydrogen solubility at $350^{\circ} \mathrm{C}$ is $126 \pm 6 \mathrm{wppm}$ and the precipitation temperature is $285^{\circ} \mathrm{C}$. These results are directly applicable to the Zry4 and ZIRLO ${ }^{\mathrm{TM}}$ samples used in the ANL test program because these samples contained $\geq 300$ wppm for Zry-4 and $\geq 350$ wppm for ZIRLO ${ }^{\text {тм }}$. However, the M $5{ }^{\circledR}$ samples tested by ANL contained lower hydrogen contents (58-94 wppm) for which total dissolution occurred during the heating ramp at temperatures in the range of $290-330^{\circ} \mathrm{C}$. Corresponding temperatures at which precipitation initiated were in the low range of $225-265^{\circ} \mathrm{C}$. Also, it was recently shown that dissolution and precipitation temperatures for $\mathrm{Nb}$ containing alloys ( $\mathrm{Zr}-1 \mathrm{Nb}$ alloy M $5 \circledR$ and $\mathrm{Zr}-1 \mathrm{Sn}-0.26 \mathrm{Nb}$ alloy N18) were essentially the same as those measured for Zry-4 [16]. More significant than alloy differences are the differences in $T_{D}$ and $T_{P}$ measured from diffusion couples $[13,14]$ and those measured using differential scanning calorimetry $[15,16]$.

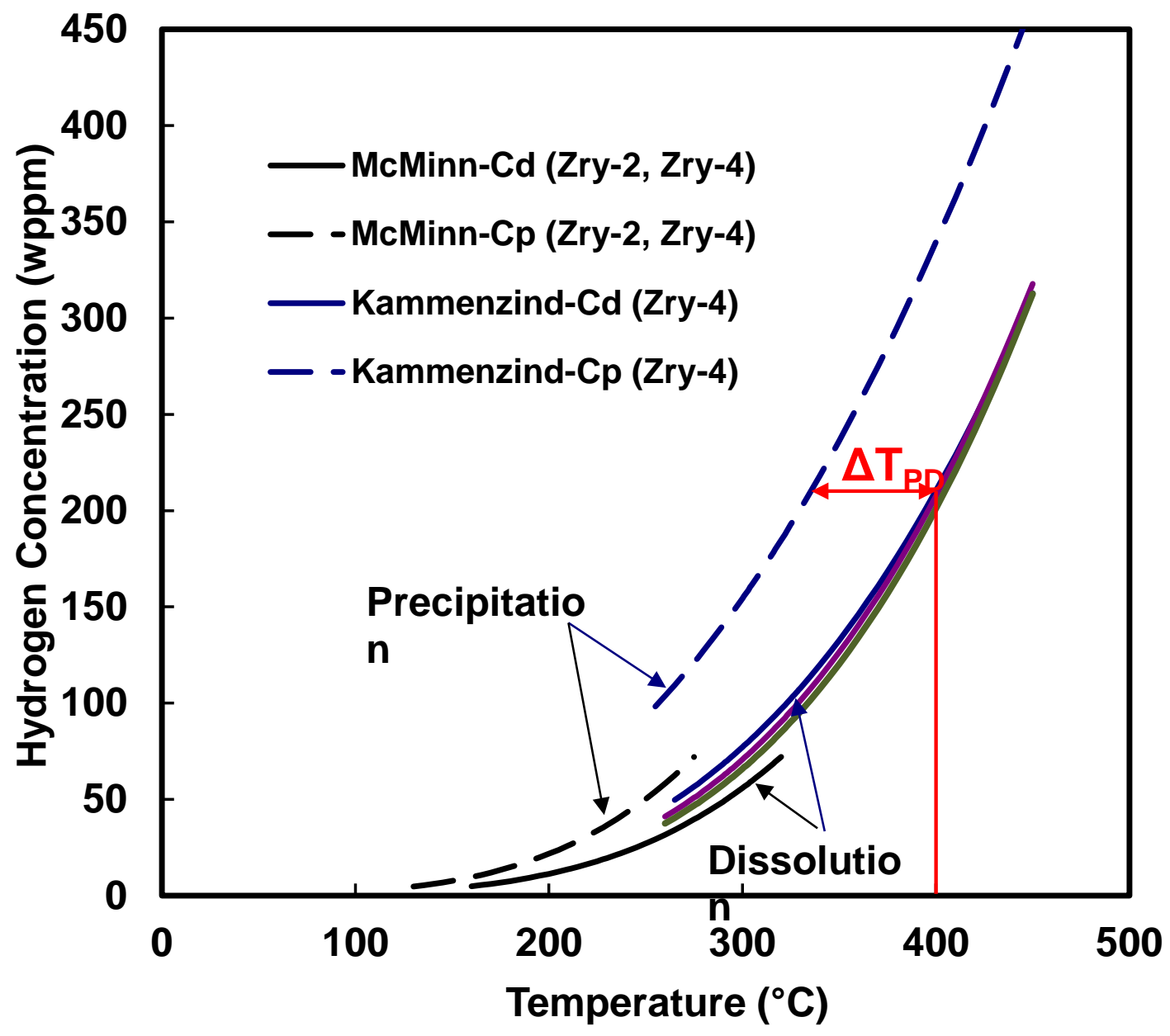

Figure 1: Steady-state curves for hydrogen dissolution and precipitation in Zr alloys. 
Page intentionally blank 


\section{HBU CLADDING MATERIALS AND TEST METHODS}

\subsection{HBU CLADDING MATERIALS}

References 9 and 10 contain detailed lists of defueled cladding materials used in previous testing. Relevant materials for the current work are listed in Table 1. The new FY2017 test is listed in bold font. For rodlets subjected to three drying cycles, the hold time at PCT refers to the hold time per cycle. The materials came from fuel rods irradiated to HBU in commercial pressurized water reactors (PWRs). The M $5{ }^{\circledR}$ cladding segment listed at $63 \mathrm{GWd} / \mathrm{MTU}$ came from a fuel rod irradiated in one of the Ringhals reactors. The other cladding segments came from two fuel rods irradiated to $68-70 \mathrm{GWd} / \mathrm{MTU}$ in the same assembly in the North Anna reactors. ZIRLO ${ }^{\mathrm{TM}}$ (now ZIRLO ${ }^{\circledR}$ ) cladding segments came from three fuel rods irradiated in the same assembly in the North Anna reactors. The $\pm C_{H}$ values represent one standard deviation in data collected from multiple axial locations along each segment and quarter-ring samples at each axial location. The large one-sigma values were due to circumferential variation in $\mathrm{C}_{\mathrm{H}}$, especially for average $C_{H}$ values $>350 \mathrm{wppm}$. Additional characterization results are presented in subsequent sections.

Table 1 Summary of $\mathrm{HBU} M 5^{\circledR}$ and ZIRLO ${ }^{\mathrm{TM}}$ cladding materials used in studies of cladding ductility following simulated drying and storage RHT at PCT.

\begin{tabular}{|c|c|c|c|c|c|c|}
\hline $\begin{array}{l}\text { Cladding } \\
\text { Alloy }\end{array}$ & TMT & $\begin{array}{l}\text { Burnup, } \\
\text { GWd/MTU }\end{array}$ & $\begin{array}{c}\text { Hydrogen } \\
\text { Content, wppm }\end{array}$ & $\begin{array}{c}\text { Peak RHT Stress, } \\
\text { M Pa }\end{array}$ & $\mathrm{PCT},{ }^{\circ} \mathrm{C}$ & $\begin{array}{l}\text { Hold Time } \\
\text { (cycles), h }\end{array}$ \\
\hline \multirow{4}{*}{ M5® } & \multirow[t]{4}{*}{ RXA } & 63 & $94 \pm 6$ & 142 & 400 & 1 (1) \\
\hline & & 68 & $72+10$ & 111 & 400 & 1 (1) \\
\hline & & 68 & $58+15$ & 90 & 400 & $1(1)$ \\
\hline & & 70 & $80 \pm 7$ & 89 & 350 & $1(1)$ \\
\hline \multirow[t]{9}{*}{$Z^{Z R L O}{ }^{T M}$} & \multirow[t]{9}{*}{ CWSRA } & 70 & $425 \pm 63$ & 111 & 400 & $1(1)$ \\
\hline & & 70 & $350 \pm 80$ & 111 & 400 & $24(1)$ \\
\hline & & 66 & $387 \pm 72$ & 87 & 350 & $24(1)$ \\
\hline & & 68 & $530 \pm 100$ & 89 & 400 & $1(1)$ \\
\hline & & 68 & $480 \pm 131$ & 88 & 400 & $1(3)$ \\
\hline & & 66 & TBD & 93 & 350 & 24 (1) \\
\hline & & 68 & $564 \pm 177$ & 93 & 350 & $1(3)$ \\
\hline & & 68 & $644 \pm 172$ & 94 & 350 & $1(1)$ \\
\hline & & 68 & $535 \pm 50$ & 80 & 400 & $1(1)$ \\
\hline
\end{tabular}

\subsection{TEST METHODS}

The protocol for single-cycle heating-cooling tests consisted of two steps: (a) simulated drying and storage testing RHT during which a sealed, pressurized rodlet is heated to and stabilized at the PCT within one hour, held at the PCT for $1-24$ hours, cooled slowly at $5^{\circ} \mathrm{C} / \mathrm{h}$ to $200^{\circ} \mathrm{C}\left(\approx 130^{\circ} \mathrm{C}\right.$ for low $-\mathrm{C}_{\mathrm{H}}$ $\mathrm{M} 5 \AA$, and cooled at a higher rate to room temperature (RT) and (b) ring-compression testing at three to four temperatures from RT to $200^{\circ} \mathrm{C}$ and at $5 \mathrm{~mm} / \mathrm{s}$ (reference value) displacement rate to a maximum sample displacement $\left(\delta_{\max }\right)$ of $1.7 \mathrm{~mm}$. For three-cycle heating-cooling RHTs, rodlets were heated to the PCT, held at the PCT for one hour, cooled at $5^{\circ} \mathrm{C} / \mathrm{h}$ to $100^{\circ} \mathrm{C}$ below the PCT, reheated to the PCT, held at PCT for one hour, cooled at $5^{\circ} \mathrm{C} / \mathrm{h}$ to $100^{\circ} \mathrm{C}$ below the PCT, reheated to the PCT, held at the PCT for one hour, and then cooled at the same rate used for the single-cycle tests. For the last three ZIRLO ${ }^{\mathrm{TM}}$ rodlets 
(105F, 646D, and $646 \mathrm{C}$ ) subjected to $350^{\circ} \mathrm{CPCT}$, the $5^{\circ} \mathrm{C} / \mathrm{h}$ cooling rate was maintained down to $130^{\circ} \mathrm{C}$ to give the dissolved hydrogen ( $\approx 125 \mathrm{wppm})$ more time to precipitate.

HBU cladding segments were used to fabricate sealed and pressurized (with argon) rodlets. Details of rodlet fabrication are given in Ref. 10. However, some details are worth repeating as fabrication problems were encountered in fabricating rodlet $(646 \mathrm{C})$ in FY2017. Following outer- and inner-surface oxide removal at the ends of the samples, as well as squaring of the ends, the components to be assembled are shown in Fig. 2. From left to right, these components include the solid bottom end fixture, the zirconia pellet used to reduce gas volume and stored energy, the high-burnup cladding segment, and the top end fixture, which has a small hole at the top to allow pressurization. In general, the fabrication sequence consists of circumferential welding of the bottom end plug, loading of the pellet, circumferential welding of the top end plug, pressurization in a chamber, laser-welding the top end-fixture hole with the rodlet in the chamber, and checking to ensure that the rodlet is indeed sealed and holds pressure. Prior to insertion of the rodlet into the RHT furnace, the rodlet is shaken several times to give a qualitative indication that internal pressure was maintained. If the zirconia pellet does not move back and forth, there has to be internal pressure inside the rodlet. Previously, it had been demonstrated that an absolute pressure of 4.7 M Pa was sufficient to prevent pellet movement. Quite recently, it was demonstrated that absolute pressure of $3.1 \mathrm{MPa}$ is insufficient to prevent rapid pellet movement during the shake test while an internal pressure of 3.6 M Pa is insufficient to prevent slow pellet movement. The shake test is also repeated after the RHT to ensure pressurization throughout the RHT. However, this qualitative test only ensures that the internal pressure is $>3.6 \mathrm{M} \mathrm{Pa}$, which should be sufficient as it is likely that defective rodlets would fully depressurize to $0.1 \mathrm{M} \mathrm{Pa}$ if there were a small flaw (e.g., pin hole or hair-line through-wall crack) in one of the welds.

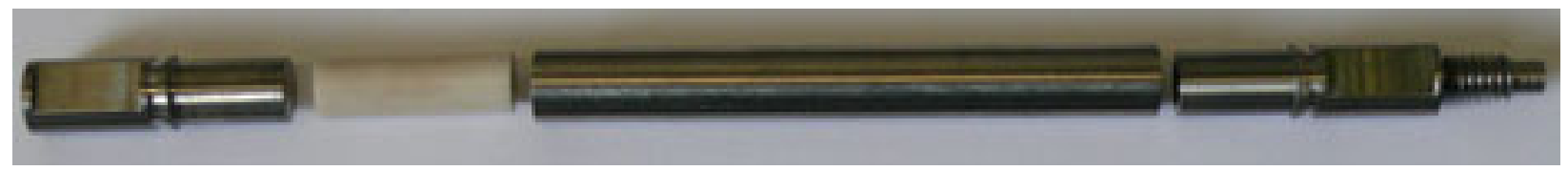

Figure 2: Rodlet components: bottom end fixture, zirconia pellet, cladding segment, and top end fixture.

Prior to rodlet pressurization, the outer diameter is measured for each cladding segment at two orientations $\left(90^{\circ}\right.$ apart) and at three axial locations. These values are averaged to give the cladding outer diameter $\left(D_{0}\right)$. The thickness of the outer-surface oxide layer $\left(h_{o x}\right)$ is estimated from sibling rod data or from interpolation or extrapolation of data from the same fuel rod at different axial locations. The same approach is used to estimate the cladding alloy wall thickness $\left(\mathrm{h}_{\mathrm{m}}\right)$. The outer diameter of the cladding alloy $\left(D_{m o}\right)$ is calculated from $D_{0}-2 h_{o x}$, and the cladding alloy inner diameter $\left(D_{m i}\right)$ is calculated from $D_{m o}-2 h_{m}$. The ratio $R_{m i} / h_{m}$, where $R_{m i}$ is the cladding alloy inner radius, is used in Eq. 1 to calculate the average hoop stress $\left(\sigma_{\theta}\right)$ from the pressure difference $\left(\Delta p=p_{i}-p_{0}\right)$ across the cladding wall, where $p_{i}$ and $p_{0}\left(0.1 \mathrm{M} \mathrm{Pa}\right.$ during fabrication at $23^{\circ} \mathrm{C}$ and $0.17 \mathrm{MPa}$ in the $\mathrm{RHT}$ furnace) are internal and external pressures, respectively.

$$
\sigma_{\theta}=\left(R_{m i} / h_{m}\right) \Delta p-p_{0}
$$

The ideal gas law is used to relate $p_{i}$ at the $P C T$ to $p_{i}$ at $23^{\circ} \mathrm{C}$ according to $p_{i}(P C T)=([P C T+273 K] / 296 K)$ $\mathrm{p}_{\mathrm{i}}\left(23^{\circ} \mathrm{C}\right)$. Given the target $\sigma_{\theta}$ at the $\mathrm{PCT}$, the fabrication pressure at $23^{\circ} \mathrm{C}$ can be calculated using Eq. 1 .

Following RHT, the rodlet is depressurized and sectioned for $\mathrm{C}_{H}$ samples, RCT samples, and metallographic imaging samples, from which precise values of the geometrical parameters in Eq. 1 can 
be determined. Using this procedure, the calculated peak rodlet $\sigma_{\theta}$ has been found to be within $\pm 3 \mathrm{MPa}$ of the target value.

The second phase of the test protocol consists of RCTs. Figure 3 shows a schematic of RCT loading. The RCT load induces maximum hoop bending stresses $\left(\sigma_{\theta}\right)$ at the inner surfaces of the 12 (under load) and 6 (above support) o'clock positions. Tensile hoop stresses also occur at the 3 and 9 o'clock outer surfaces. Associated with these tensile stresses are tensile strains $\left(\varepsilon_{\theta}\right)$. Within the elastic range, hoop stresses at 3 and 9 o'clock are about $40 \%$ less than hoop stresses at 12 and 6 o'clock. Also, because the length (L $\approx 8$ $\mathrm{mm}$ ) of the rings is much greater than the cladding wall thickness ( 0.54 to $0.57 \mathrm{~mm}$ for HBU ZIRLO ${ }^{\mathrm{TM}}$ and $\mathrm{M} 5 \AA$, an axial stress is induced that is 0.37 times the hoop stress within the elastic deformation regime. The maximum sample displacement $\left(\delta_{\max }=1.7 \mathrm{~mm}\right)$ is chosen to give $\approx 10 \%$ offset strain at RT. The starting point for the RCT is $1 \mathrm{~mm}$ above the sample to allow the full displacement rate to develop. The reference displacement rate is $5 \mathrm{~mm} / \mathrm{s}$.

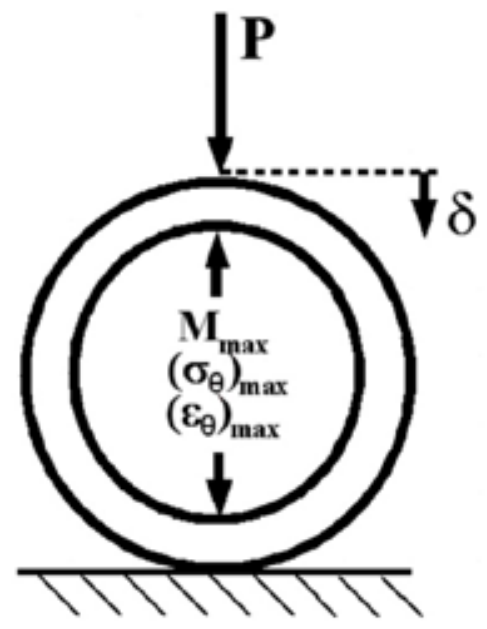

Figure 3: RCT measured load (P) and controlled displacement ( $\delta)$.

Load-displacement curves and post-test diameter measurements are used to determine offset $\left(\delta_{p}\right)$ and permanent $\left(d_{p}\right)$ displacements, respectively. These are normalized to $D_{m o}$ to give relative plastic displacement (i.e., plastic strain) for the ring structure. Permanent displacement is defined as the difference between pre- and post-test diameter measurements along the loading direction. Figure 4 shows how traditional and corrected offset displacements are determined from a benchmark loaddisplacement curve for an as-fabricated (AF) $17 \times 17 \mathrm{M} 5{ }^{\circledR}$ ring. For the benchmark sample, $\mathrm{D}_{\mathrm{mo}}=9.48$ $\mathrm{mm}, \mathrm{h}_{\mathrm{m}}=0.61 \mathrm{~mm}$, and $\mathrm{L}=8.02 \mathrm{~mm}$. The traditional offset-displacement methodology calls for unloading the sample at the same slope as the measured linearized loading slope $\left(K_{L M}\right)$. It should be noted that $K_{L M}$ is less than the calculated sample stiffness $\left(K_{L C}\right)$ due to the influence of machine compliance. This approach gives a traditional $\delta_{p}=1.21 \mathrm{~mm}$, which is greater than the more accurate $d_{p}=$ $1.07 \mathrm{~mm}$. Thus, there is an inherent error in the traditional approach as the measured linearized unloading slope $\left(\mathrm{K}_{U M}\right)$ is always less than $\mathrm{K}_{\mathrm{LM}}$. $\mathrm{K}_{\mathrm{UM}}$ is determined from the slope of the line connecting $\delta_{\max }$ (plus $1 \mathrm{~mm}$ ) to the displacement axis value based on the measured value of $d_{p}$ at zero load. Normalizing these displacements to $D_{\text {mo }}$ gives $12.8 \%$ traditional offset strain and $11.3 \%$ permanent strain, which is also the "corrected offset strain" for these benchmark tests. 


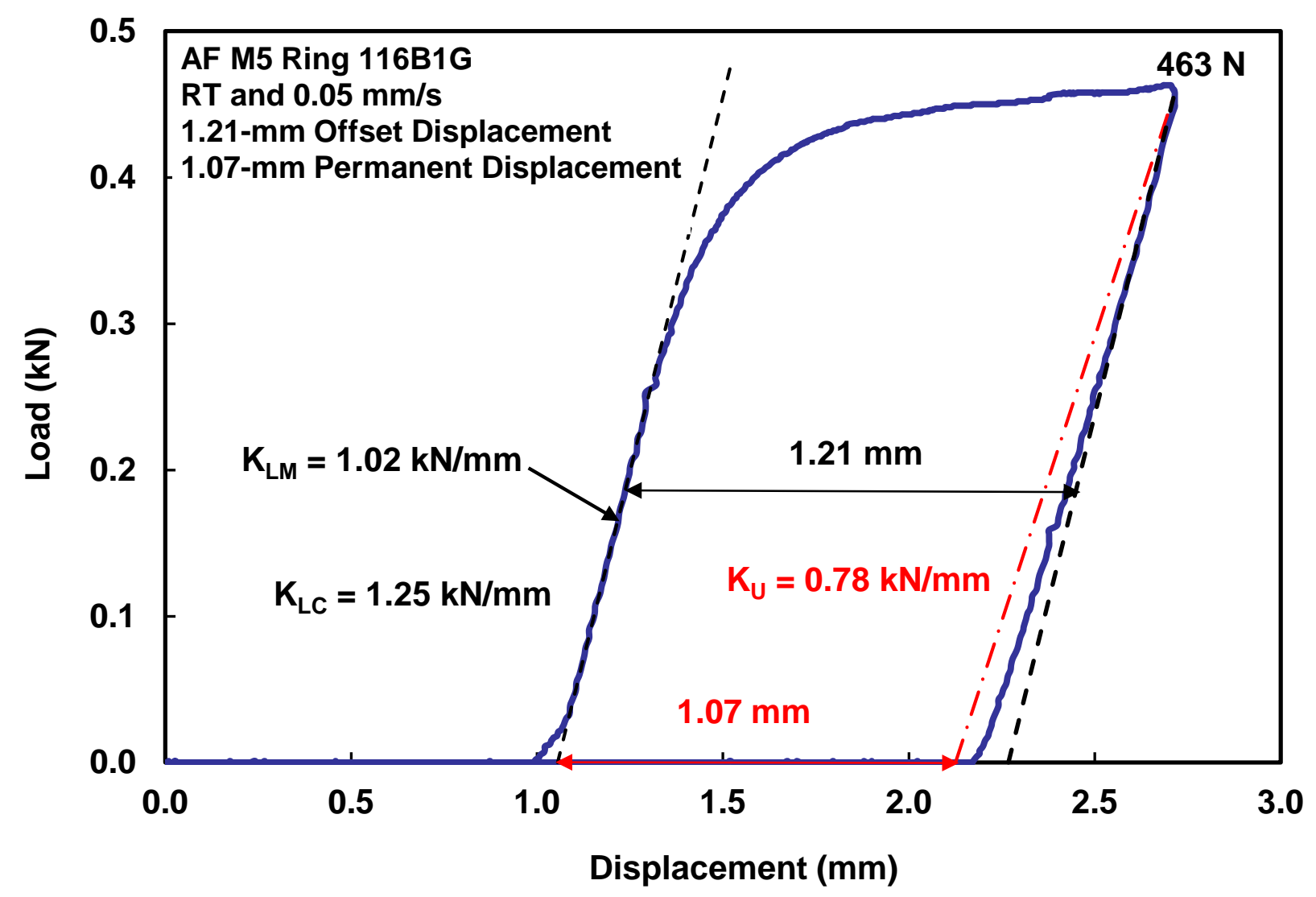

Figure 4: Load-displacement curve for as-fabricated (AF) M5 ${ }^{\circledR}$ ring tested at RT and $0.05 \mathrm{~mm} / \mathrm{s}$ to $1.7 \mathrm{~mm}$ ring displacement.

Energy methods were used to determine the bending moment as a function of circumferential orientation. Wide-beam theory (WBT) was used to relate the bending moment to bending stresses and strains within the elastic-deformation regime, as well as the relationship between the elastic load $\left(\mathrm{P}_{\mathrm{e}}\right)$ and elastic displacement $\left(\delta_{e}\right)$. The curvature of the ring was not taken into account in the bending analysis. The calculated loading stiffness is $\mathrm{K}_{\mathrm{LC}}=\mathrm{P}_{\mathrm{e}} / \delta_{\mathrm{e}}$, where

$$
P_{e}=\left\{(E L) /\left[1.79\left(1-v^{2}\right)\right]\right\}\left(h_{m} / R_{\text {mid }}\right)^{3} \delta_{e}
$$

and $E$ is Young's modulus, $v$ is Poisson's ratio, and $R_{\text {mid }}=\left(D_{m o}-h_{m}\right) / 2$ is the mid-wall radius. On the basis of finite element analysis (FEA) results, the loading stiffness calculated using WBT (Eq. 2) is about $7 \%$ higher than the FEA-calculated loading stiffness. However, Eq. 2, which is a closed form solution, is useful for relating $P_{\max }$ in the plastic deformation regime to geometrical parameters. The expectation is that $P_{\max }$ is proportional to $L$ and to $\left(h_{m} / R_{\text {mid }}\right)^{n}$, where $n$ is $<3$.

For HBU cladding rings that crack during the 1.7- $\mathrm{mm}$ displacement, $\mathrm{d}_{\mathrm{p}}$ cannot be determined accurately. Thus, one must rely on a correlation for the unloading slope to determine the corrected offset displacement prior to the first significant crack, from which the ductility can be determined. The correlation developed for this application is based on the results from a large number of benchmark tests with permanent displacements ranging from $0.14 \mathrm{~mm}$ to $1.2 \mathrm{~mm}$, displacement rates in the range of $0.03-50 \mathrm{~mm} / \mathrm{s}$, and temperatures in the range of $20-150^{\circ} \mathrm{C}$. Results of these benchmark tests, including tests conducted in FY2017, are shown in Fig. 5 in terms of the ratio of measured unloading/loading $\left(\mathrm{K}_{U M} / \mathrm{K}_{L M}\right)$ slopes vs. traditional offset strain $\left(\delta_{\mathrm{p}} / \mathrm{D}_{\mathrm{mo}}\right)$. Also shown in Fig. 5 are results 
from nine RCTs with $\mathrm{HBU} M 5{ }^{\circledR}$ (solid red circles) that exhibited no cracking after 1.7-mm total displacement. The hollow blue-circle data points are from RCTs conducted with AF $17 \times 17$ M $5 \circledR(40$ points) and AF $17 \times 17 \mathrm{ZIRLO}^{\mathrm{TM}}$ (6 points) cladding samples. Outer diameters were $9.49 \pm 0.1 \mathrm{~mm}$ and nominal cladding wall thickness values were $0.61 \mathrm{~mm}$ (reference case) and $0.51 \mathrm{~mm}$ (6 ZIRLO ${ }^{\mathrm{Tm}}$ data points and $5 \mathrm{M} 5{ }^{\circledR}$ data points). The data set also includes RCT results from two machines: (a) Instron 5556 and (b) Instron 8511.

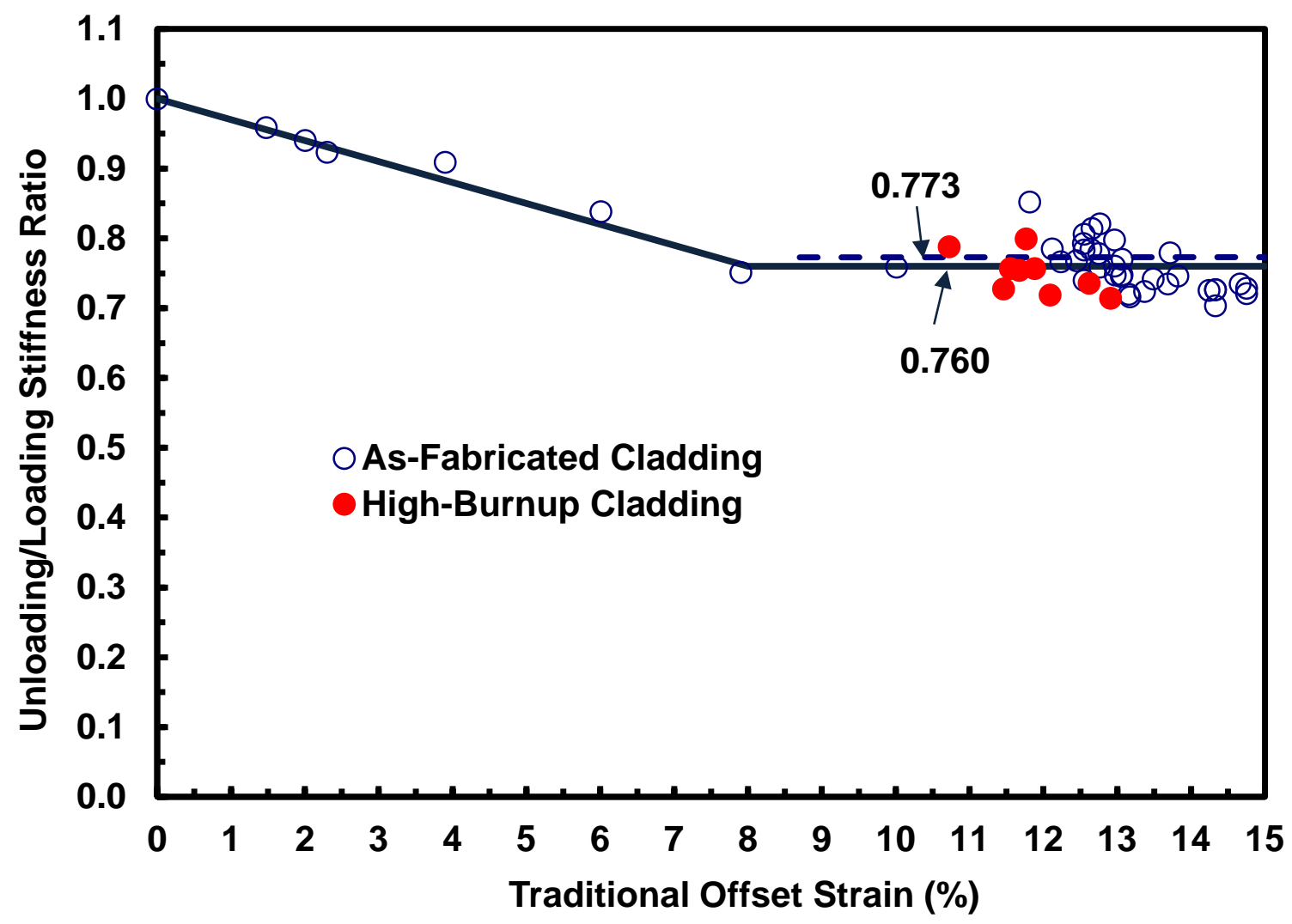

Figure 5: RCT benchmark results for determining the ratio of unloading/loading stiffness as a function of the traditional offset strain.

The correlation developed from an earlier subset of the benchmark-test data for the calculated linearized unloading slope ( $\mathrm{K}_{U}$ in $\mathrm{kN} / \mathrm{mm}$ ) as a function of the measured loading slope (in $\mathrm{kN} / \mathrm{mm}$ ) and the measured traditional offset strain (in \%) is:

$$
\begin{aligned}
& K_{U} / K_{L M}=1-0.02612 \delta_{p} / D_{m o} \text { for } \delta_{p} / D_{m 0} \leq 8.7 \% \\
& K_{U} / K_{L M}=0.773 \text { for } \delta_{p} / D_{m 0}>8.7 \%
\end{aligned}
$$

For the complete data set shown in Fig. 5, the stiffness ratio is 0.760 at traditional offset strains $>8 \%$. The best-fit slope at $\leq 8 \%$ traditional offset strain is 0.03013 as compared to 0.02612 in Eq. 3a. As these differences are insignificant, corrected offset strains continue to be determined using $\mathrm{K}_{\mathrm{u}}$ calculated by Eqs. $3 a$ and $3 b$. 
The criteria for determining embrittlement remain the same for cladding with radial and circumferential hydrides: corrected $\delta_{\mathrm{p}} / \mathrm{D}_{\mathrm{mo}}<2 \%$ prior to $>25 \%$ load drop or $>50 \%$ decrease in loading slope. In previous work [4-7], it was established that $>25 \%$ load drop and $>50 \%$ decrease in loading slope correspond to a crack or cracks extending through $>50 \%$ of the wall thickness. In a few cases, an "implied" load drop $>25 \%$ is used. For these cases, cracking initiates at loads much less that the $P_{\max }$ measured for rings that do not exhibit early cracking. Although the explicit load drops appear to be $<25 \%$ followed by a smooth load-displacement curve, the $P_{\max }$ may be $30 \%$ to $50 \%$ less than expected.

The $2 \%$ offset strain limit is based on the uncertainty in measurement of the permanent strain $\left(d_{p} / D_{m o}\right.$ $\leq 1 \%)$ for as-fabricated cladding and the added uncertainty $(\leq 1 \%)$ in the permanent displacement for HBU cladding due to flaking off of the corrosion (i.e., oxide) layer under the applied load and above the support plate. Multiple cracks through the oxide layer and the hydride rim lower the unloading stiffness to values less than shown in Fig. 5 . As the $\mathrm{HBU}^{\mathrm{M} 5}{ }^{\circledR}$ tested had very thin oxide layers $(\approx 10 \mu \mathrm{m})$ and no hydride rim, the $2 \%$ offset strain criterion is more conservative for this HBU alloy than for the HBU Zry-4 and $\mathrm{ZIRLO}^{\mathrm{TM}}$ samples tested, both of which had thicker oxide layers $(30-100 \mu \mathrm{m})$ and thick hydride rims $(30-100 \mu \mathrm{m})$.

In previous testing of benchmark and $\mathrm{HBU}$ cladding samples, the $\mathrm{P}_{\max }$ was noted on load-displacement curves and used to verify the load-cell calibration. However, $P_{\max }$ can also be used to assess the degree of irradiation hardening in HBU cladding and possible short-time annealing during RHT if results can be normalized to the geometrical parameters $L, h_{m}$, and $R_{\text {mid. }}$. As mentioned earlier, the normalization factor is expected to be of the form $L\left(h_{m} / R_{\text {mid }}\right)^{n}$. Additional benchmark tests were conducted during this reporting period with $0.61-\mathrm{mm}$ and $0.57-\mathrm{mm}$ wall-thickness AF M $5 \AA$ samples to determine the exponent " $n$ ". The reference value of $L$ was $8 \mathrm{~mm}$ and the reference value of $h_{m} / R_{\text {mid }}$ was 0.0689 . As shown in Table 2, $n=2.44$ resulted in excellent agreement between normalized $P_{\max }$ values for thin- and thick-wall cladding samples.

Table 2 RCT benchmark results for $17 \times 17$ AF M5 ${ }^{\circledR}$ cladding with wall thicknesses of $0.61 \mathrm{~mm}$ and $0.57 \mathrm{~mm}$. The maximum load in the last column is normalized by $(8.0-\mathrm{mm} / \mathrm{L})$ $\left(0.0689 /\left[h_{m} / R_{\text {mid }}\right]\right)^{2.44}$.

\begin{tabular}{|c|c|c|c|c|c|}
\hline $\mathrm{T},{ }^{\circ} \mathrm{C}$ & $\begin{array}{l}\mathrm{d} \delta / \mathrm{dt} \\
\mathrm{mm} / \mathrm{s}\end{array}$ & $\begin{array}{l}\text { Number of } \\
\text { Data Points }\end{array}$ & $(8-m m / L) P_{\max }, N$ & $\left(h_{m} / R_{\text {mid }}\right)$ & $\begin{array}{c}\text { Normalized } \mathbf{P}_{\max }, \\
\mathbf{N}\end{array}$ \\
\hline 25 & 0.05 & 9 & $467 \pm 5$ & $0.0689 \pm 0.0003$ & $467 \pm 5$ \\
\hline 25 & 0.05 & 3 & $390 \pm 3$ & 0.0640 & $466 \pm 4$ \\
\hline 25 & 5 & 13 & $512 \pm 5$ & $0.0698 \pm 0.0010$ & $496 \pm 5$ \\
\hline 25 & 50 & 1 & 535 & 0.0705 & 506 \\
\hline 120 & 0.05 & 5 & $360 \pm 2$ & $0.0689 \pm 0.0003$ & 360 \\
\hline 120 & 0.05 & 1 & 302 & 0.0638 & 363 \\
\hline 120 & 5 & 1 & 417 & 0.0690 & 416 \\
\hline 120 & 5 & 1 & 348 & 0.641 & 414 \\
\hline
\end{tabular}

The geometrical normalization factor allows comparison between $\mathrm{P}_{\max }$ data for AF M 5 ®and $\mathrm{P}_{\max }$ data for HBU M $5 \circledR$ to assess irradiation hardening for as-irradiated cladding and possible annealing during hold times at the PCT. For four sets of HBU M $5{ }^{\circledR R C T}$ data, $L$ was $7.88 \pm 0.12 \mathrm{~mm}, \mathrm{~h}_{\mathrm{m}}$ was $0.559 \pm 0.0100 \mathrm{~mm}$ 
and $D_{\text {mo }}$ was $9.51 \pm 0.01 \mathrm{~mm}$. Results are discussed in Section 5 and compared to published data for the increases in yield and ultimate tensile stresses for HBU M 5®. During FY2018, additional benchmark tests will be conducted with $0.57-\mathrm{mm}$-wall AF M $5{ }^{\circledR}$, which is closer in thickness to the HBU M $5 \circledR$ tested than the $0.61-\mathrm{mm}$-wall M $5 \circledR$ used for most of the benchmark tests.

The data reported in Table 2 was generated from 2011-2017 mostly using the Instron 8511. Tests conducted with the Instron 5566 are important for verifying the calibration of the Instron 8511. However, it was found that Instron 5566 compression tests conducted at $5 \mathrm{~mm} / \mathrm{s}$ resulted in enough displacement overshoot so that $d_{p}$ was greater than or equal to $\delta_{p}$, which is inconsistent with all previous data. For a screw type machine used in compression, it is very difficult for the machine to stop precisely at the specified displacement limit when set to high displacement rates. Even for the slower displacement rate $(0.05 \mathrm{~mm} / \mathrm{s})$ there was some evidence of overshoot, which has a small and insignificant effect on $P_{\max }$ and a very significant effect on the difference between $d_{p}$ and $\delta_{p}$. The 10year-old software and computer will be upgraded in FY2018 before additional benchmark tests are conducted with the Instron 5566. 
Page intentionally blank 


\section{PREVIOUS RESULTS FOR HBU M5 ${ }^{\circledR}$ AND HBU ZIRLO ${ }^{\text {TM }}$}

\subsection{HBU M5 ${ }^{\circledR}$}

Figure 6 summarizes the RCT ductility data measured for as-irradiated HBU M5® and for HBU M $5 \circledR$ following cooling from peak RHT conditions of $350^{\circ} \mathrm{C}$ and $400^{\circ} \mathrm{C}$ and $89-142 \mathrm{MPa}$ hoop stresses. The asirradiated material with 76-wppm hydrogen had high ductility at RT for a wide range of displacement rates $(0.05-50 \mathrm{~mm} / \mathrm{s})$. None of these samples exhibited cracking. Similar results were obtained for HBU $\mathrm{M} 5{ }^{\circledR}$ with 58-wppm hydrogen following cooling from $400^{\circ} \mathrm{C}$ and $90-\mathrm{M} \mathrm{Pa}$ hoop stress. These results were somewhat surprising in that most of the hydrogen precipitated in the radial direction to give RHCF $=$ $37 \pm 17 \%$. It was hypothesized that the low $\mathrm{C}_{\mathrm{H}}$ resulted in poor connectivity or continuity of radial hydrides in the axial direction and consequently enhanced ductility. This has been supported by RCT results shown in Fig. 6 for an 80-wppm HBU M 5®rodlet following slow cooling from peak RHT conditions of $350^{\circ} \mathrm{C}$ and $89 \mathrm{M} \mathrm{Pa}$. The RHCF was $44 \pm 18 \%$ (see next section) and the DTT was about $70^{\circ} \mathrm{C}$. The RHCF increased with increasing peak hoop stress: $54 \pm 20 \%$ for $111 \mathrm{MPa}$ and $72 \mathrm{wppm}$ and $61+18 \%$ for 142 $\mathrm{MPa}$ and $94 \mathrm{wppm}$, but the DTT values for these samples were very close $\left(70-80^{\circ} \mathrm{C}\right)$. At such low hydrogen contents, peak cladding stresses are less important than the stresses at $T_{p}$, which decrease as $\mathrm{C}_{\mathrm{H}}$ decreases. Figure 7 shows the increase in $\mathrm{HBU} \mathrm{M} 5{ }^{\circledR D} \mathrm{DTT}$ with hydrogen content. Also shown in Fig. 7 are the hoop stresses (67-113 MPa) at $T_{p}$. It is clear from these results that the DTT increases with $C_{H}$ within these precipitation stress levels. The expected peak $C_{H}$ for $17 \times 17 \mathrm{M} 5 \AA$ from fuel rods irradiated in a commercial PWR for two-to-three 18-month fuel cycles is in the range of $100 \pm 20 \mathrm{wppm}$

\subsection{HBU ZIRLO ${ }^{\text {TM }}$}

HBU ZIRLO ${ }^{\text {TM }}$ was subjected to RCTs in the as-irradiated condition and following RHT at $350^{\circ} \mathrm{C}$ and $400^{\circ} \mathrm{C}$ PCT and 80-141 MPa. The hydrogen concentrations of the test samples ranged from about 350 wppm to 650 wppm. Ductility results are summarized in Fig. 8. In the as-irradiated condition HBU ZIRLO ${ }^{\mathrm{TM}}$ had relatively high ductility $(6 \%$ to $>11 \%$ ) for tests conducted at RT displacement rates of $0.05 \mathrm{~mm} / \mathrm{s}$ to 50 $\mathrm{mm} / \mathrm{s}$ and at $5 \mathrm{~mm} / \mathrm{s}$ and RT to $150^{\circ} \mathrm{C}$. For an $80-\mathrm{M} \mathrm{Pa}$ peak RHT hoop stress, radial hydrides were short $(9 \pm 4 \%$ RHCF $)$ and ductility values were moderately high at RT and $60^{\circ} \mathrm{C}$ and high $(>9 \%)$ at $90^{\circ} \mathrm{C}$ and $150^{\circ} \mathrm{C}$ $[6,9]$. Thus, for peak RHT conditions of $400^{\circ} \mathrm{C}$ and $\leq 80 \mathrm{MPa}$, the corresponding DTT would be $<20^{\circ} \mathrm{C}$. The two rodlets subjected to peak RHT conditions of $400^{\circ} \mathrm{C}$ and $111 \mathrm{MPa}$ exhibited longer radial hydrides $(32+13 \%)$ and a higher DTT value $\left(122^{\circ} \mathrm{C}\right)$ [3]. For HBU ZIRLO ${ }^{\mathrm{TM}}$ rodlets subjected to peak RHT conditions of $350^{\circ} \mathrm{C}$ and $400^{\circ} \mathrm{C}$ within the narrow stress range of $87-89 \mathrm{MPa}$, radial hydrides remained relatively short ( $\leq 20 \%$ on average) and the DTT was $<30^{\circ} \mathrm{C}$. However, for rodlets subjected to higher peak hoop stresses $\left(93-94 \mathrm{MPa}\right.$ at $350^{\circ} \mathrm{C}$ and $111 \mathrm{MPa}$ at $400^{\circ} \mathrm{C}$ ), radial hydrides were considerably longer (>30\%) and the DTT values were $>120^{\circ} \mathrm{C}$. Excluding the influence of total hydrogen content, the results suggested a very high sensitivity of ZIRLO ${ }^{\mathrm{TM}}$ to radial-hydride-induced embrittlement within the narrow stress range of $90 \pm 3 \mathrm{MPa}$. This is shown in Fig. 9 in which the DTT is plotted as a function of peak hoop stress and corresponding precipitation-temperature hoop stress. However, the higher hydrogen contents and thicker hydride rims for the 93-M Pa (564+177 wppm) and 94-M Pa $(644 \pm 172 \mathrm{wppm})$ cases may have contributed to their higher DTT values.

Additional test data are needed to confirm the high sensitivity of HBU ZIRLO ${ }^{T M}$ DTT to RHT stress (see next section) and to investigate the effects of very thick hydride rims on cladding ductility. However, the expected peak $\mathrm{C}_{H}$ for $17 \times 17$ ZIRLO ${ }^{\circledR}$ from fuel rods irradiated in a commercial PWR for two-to-three 18month fuel cycles is $\leq 550$ wppm (averaged over an axial length of about $50 \mathrm{~mm}$ ). 


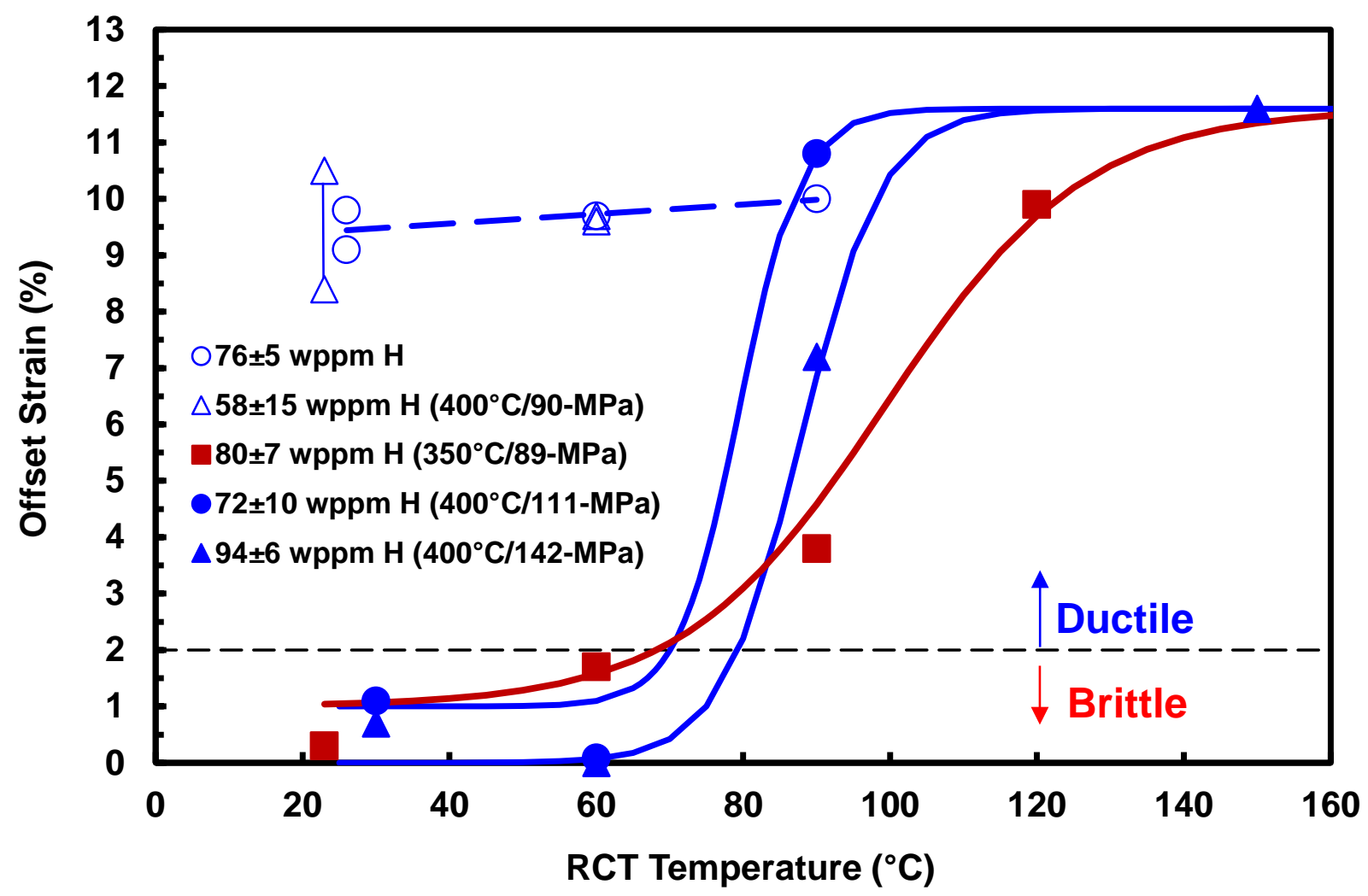

Figure 6: RCT ductility curves for $\mathrm{HBU} M 5^{\circledR}$ in the as-irradiated condition and following RHT at $350^{\circ} \mathrm{C}$ and $400^{\circ} \mathrm{C} \mathrm{PCT}$.

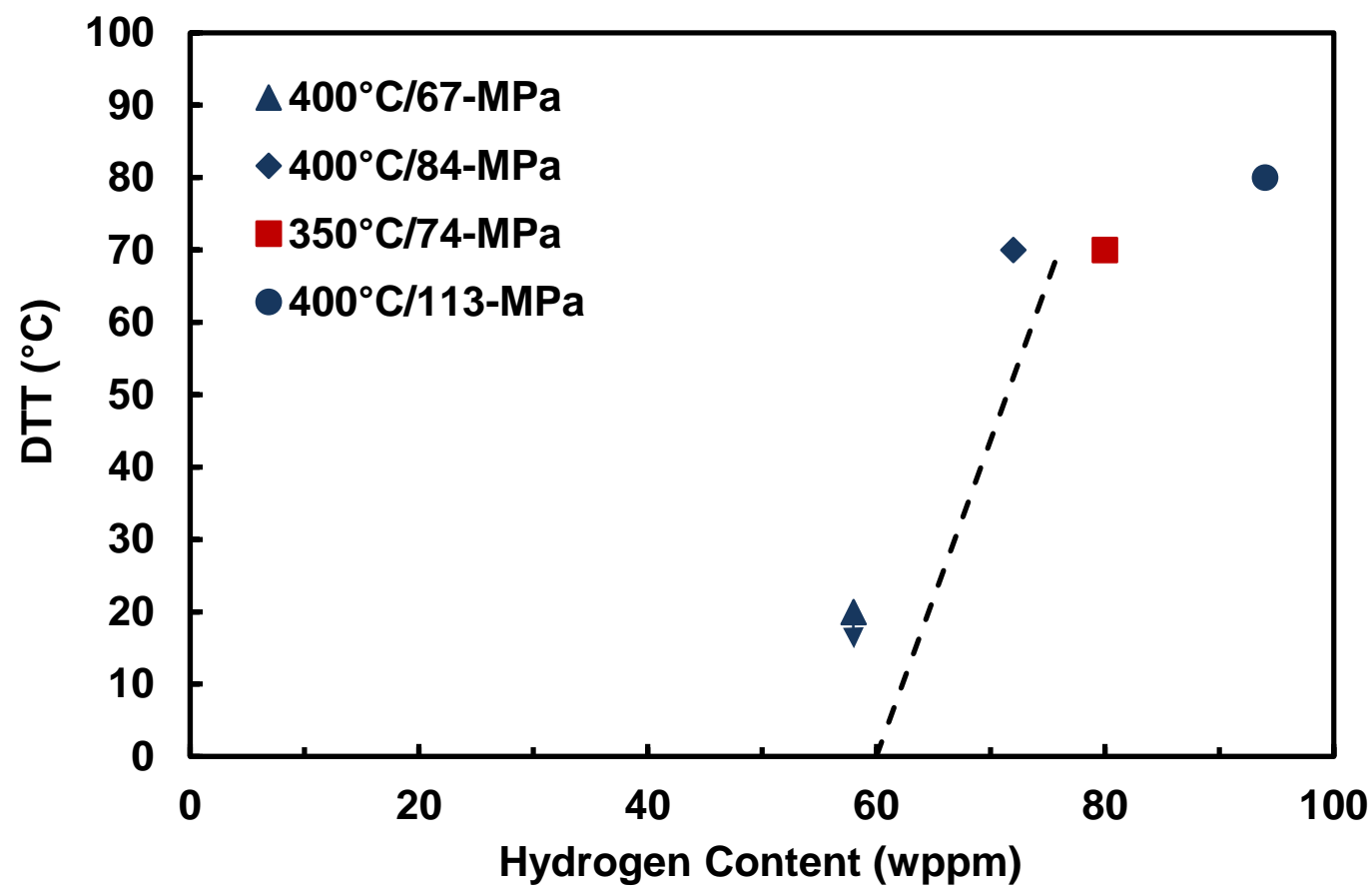

Figure 7: DTT for $\mathrm{HBU} M 5^{\circledR}$ vs. hydrogen content following $\mathrm{RHT}$ at $350^{\circ} \mathrm{C}$ and $400^{\circ} \mathrm{C}$ PCT. 


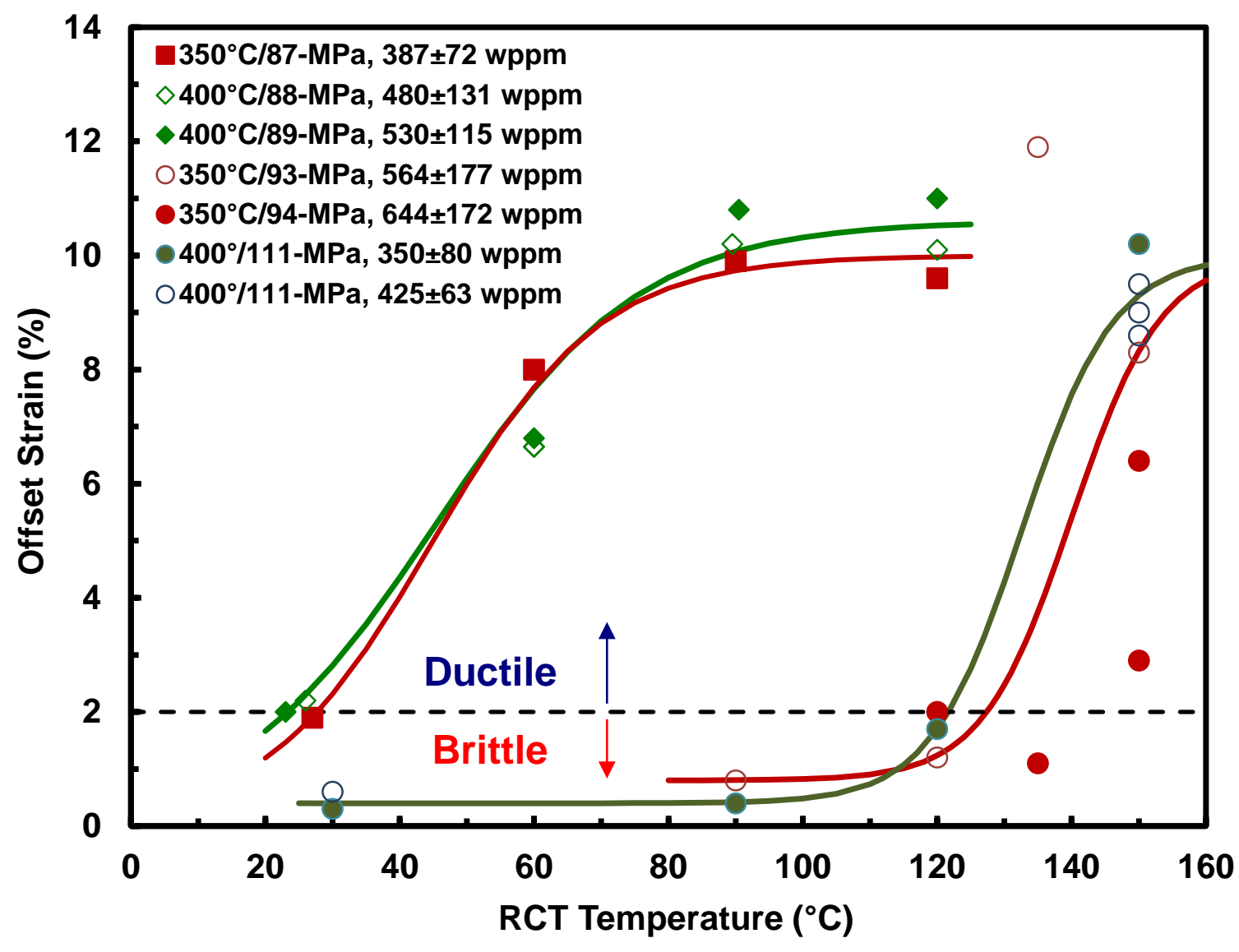

Figure 8: Summary of ductility data for HBU ZIRLO ${ }^{\mathrm{TM}}$ following RHT at $400^{\circ} \mathrm{C}$ and $350^{\circ} \mathrm{C} \mathrm{PCT}$.

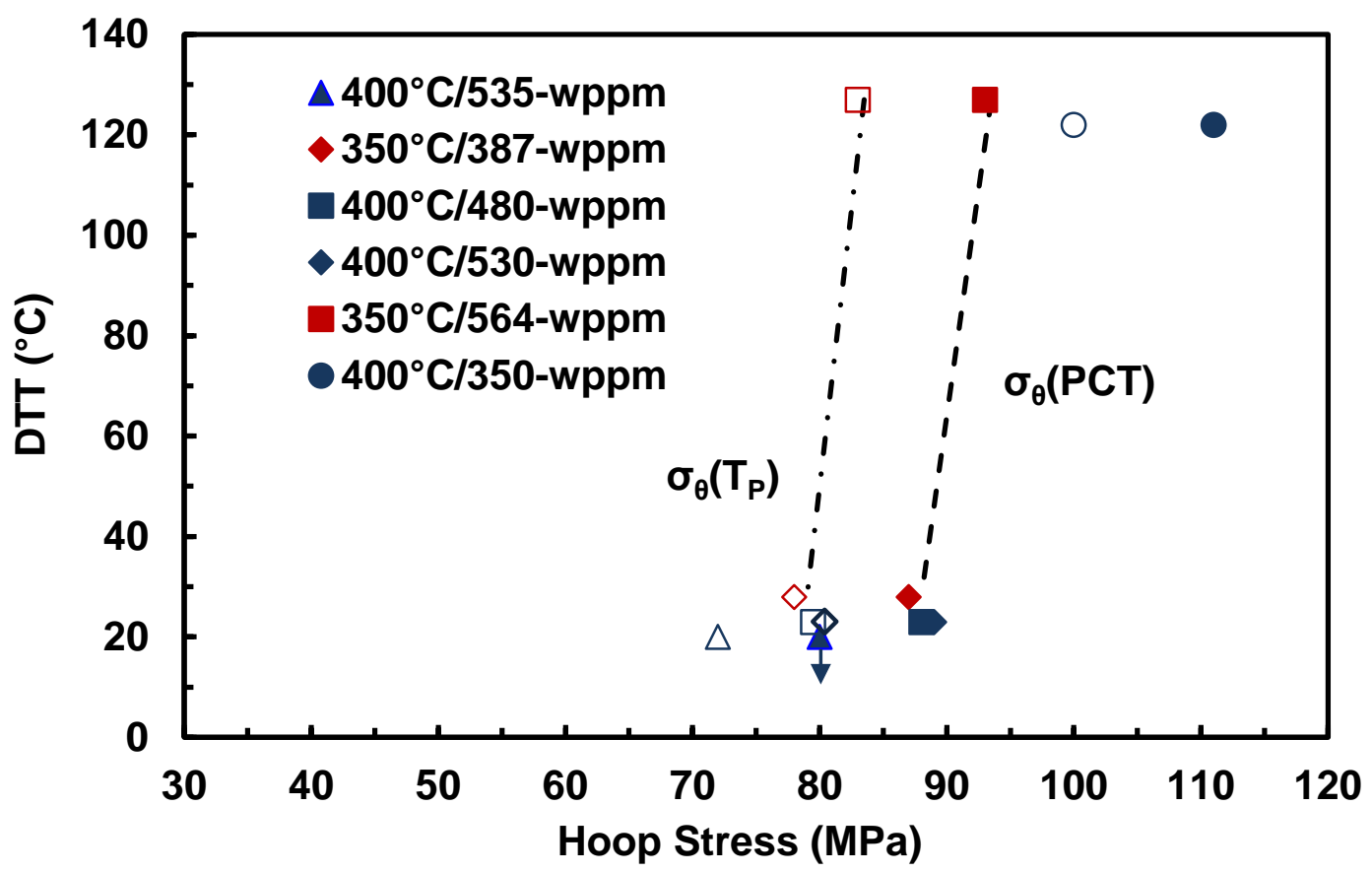

Figure 9: DTT vs. hoop stress for HBU ZIRLO ${ }^{\mathrm{TM}}$ following RHT at $400^{\circ} \mathrm{C}$ and $350^{\circ} \mathrm{C} \mathrm{PCT}$. 
Page intentionally blank 


\section{RESULTS FROM CURRENT TESTS}

\subsection{AXIAL CONTINUITY OF RADIAL HYDRIDES IN HBU M5 ${ }^{\circledR}$}

Figure 10 shows the sectioning diagram following RHT and RCT for HBU M5 ${ }^{\circledR}$ rodlet $652 \mathrm{~F} 2$ (80 \pm 7 wppm hydrogen), which had been subjected to peak RHT conditions of $350^{\circ} \mathrm{C}$ and $89 \mathrm{M} \mathrm{Pa}$ for 1 hour prior to slow cooling. Metallographic samples were prepared at the indicated surfaces of ring $G$ (left end and mid-span) and ring $\mathrm{H}$ (mid-span). On the basis of load-displacement curves, ring $\mathrm{G}$ exhibited very high ductility at $120^{\circ} \mathrm{C}$ and ring $\mathrm{H}$ exhibited brittle behavior at RT. The effective lengths (average and maximum) of radial hydrides at these three locations were determined and compared to those obtained previously [12] for sample $F$ to enable evaluation of the degree of continuity of radial hydrides in the axial direction within a span of $15 \mathrm{~mm}$. The $\mathrm{H} 1$ sample surface was also examined to determine the number and extent of cracks, as well as the direction of the cracks with respect to the cladding radius.

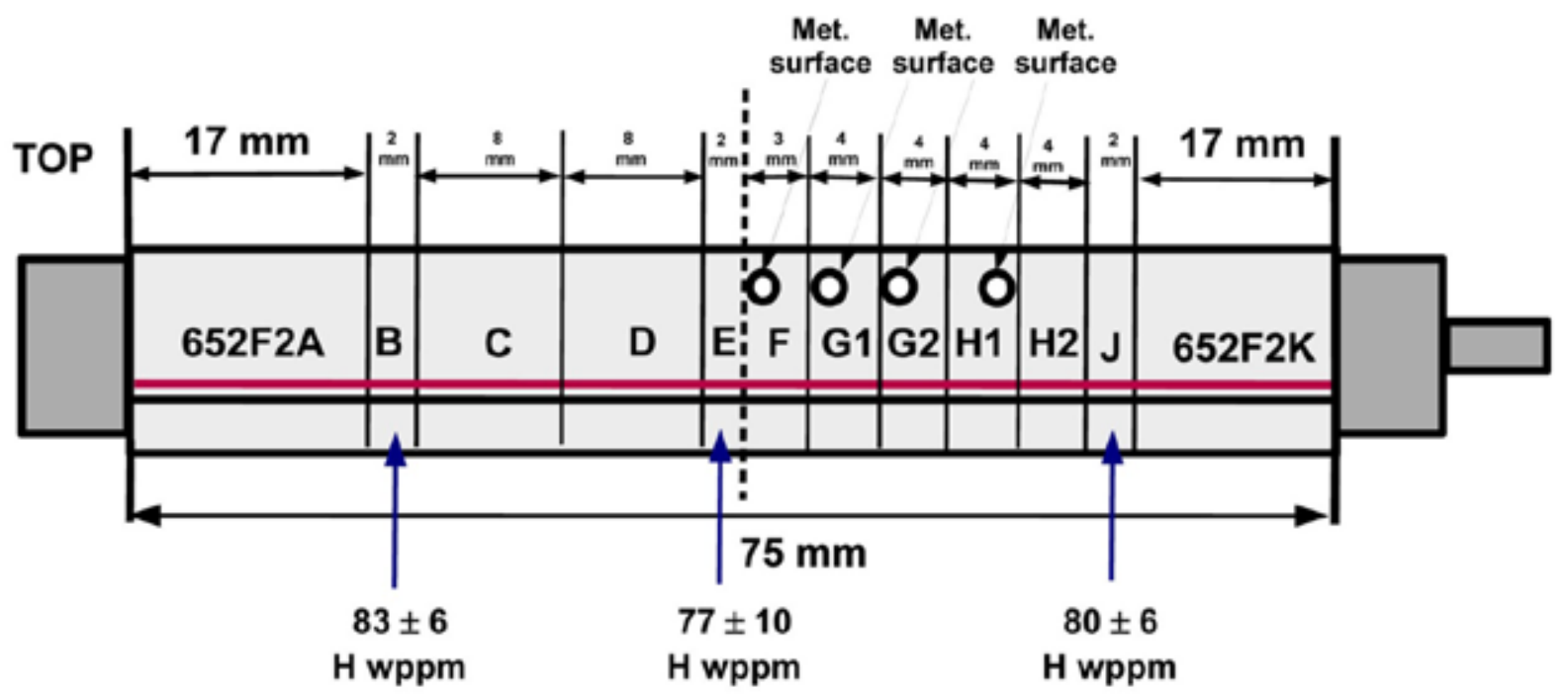

Figure 10: Sectioning diagram for $\mathrm{HBU} M 5^{\circledR}$ rodlet $652 \mathrm{~F} 2$ following $\mathrm{RHT}$ and RCT.

Three sets of metallographic images were generated for sample 652F2F to determine the effective lengths and degree of axial connectivity of radial hydrides: (a) at the indicated surface (0-mm axial location) following grinding, polishing, and etching; (b) after grinding off about $0.5 \mathrm{~mm}$ followed by polishing and etching (0.5- $\mathrm{mm}$ axial location); and after grinding off an additional $0.1 \mathrm{~mm}$ using fine grit paper followed by polishing and etching $(0.6-\mathrm{mm}$ axial location). Images were taken at enough circumferential orientations (45 for the $0-\mathrm{mm}$ location) for the 100X images to cover the whole cross section with some overlapping regions from identification purposes. Images at 200X were also taken to better determine the radial continuity of the longer hydrides. For the convenience of the reader, these images, which were shown in Ref. 12, are repeated in this section. Figures 11 a and 11b show images (reduced in size) of the 0-mm axial-location surface at 100X and 200X, respectively, of the longest radial hydride (95\% RHCF), which occurred at the 12:30 o'clock orientation. The average ( \pm one standard deviation) RHCF was $49 \pm 14 \%$. For the $652 \mathrm{~F} 2 \mathrm{~F}$ surface located at $0.5 \mathrm{~mm}$, the longest radial hydride (96\%) was found at the $3: 30$ orientation and the average RHCF was $54 \pm 18 \%$. Figure 12 shows the $100 \mathrm{X}$ metallographic image of the longest radial hydride at the $0.5-\mathrm{mm}$ axial location. 


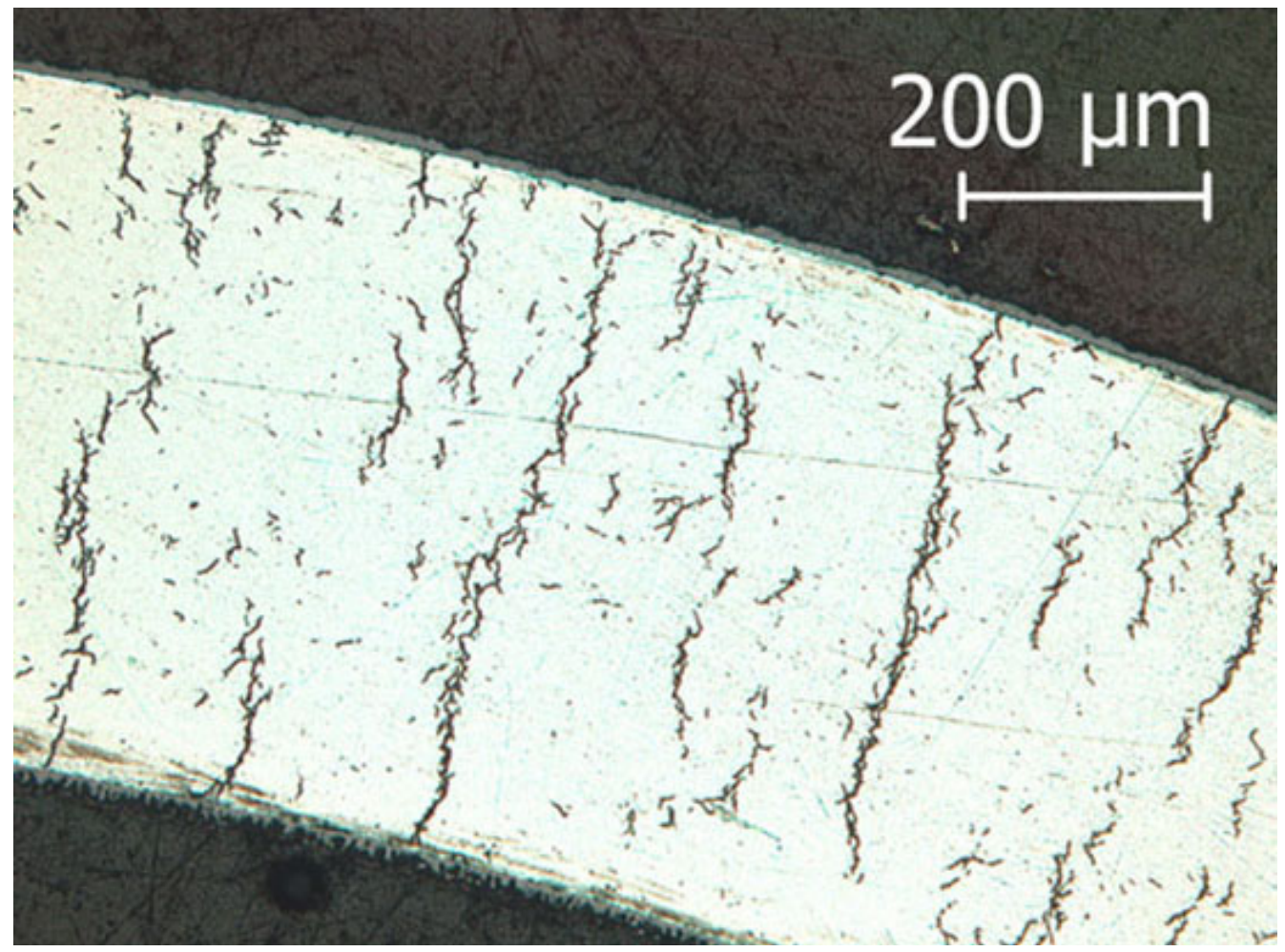

(a) $100 \mathrm{X}$

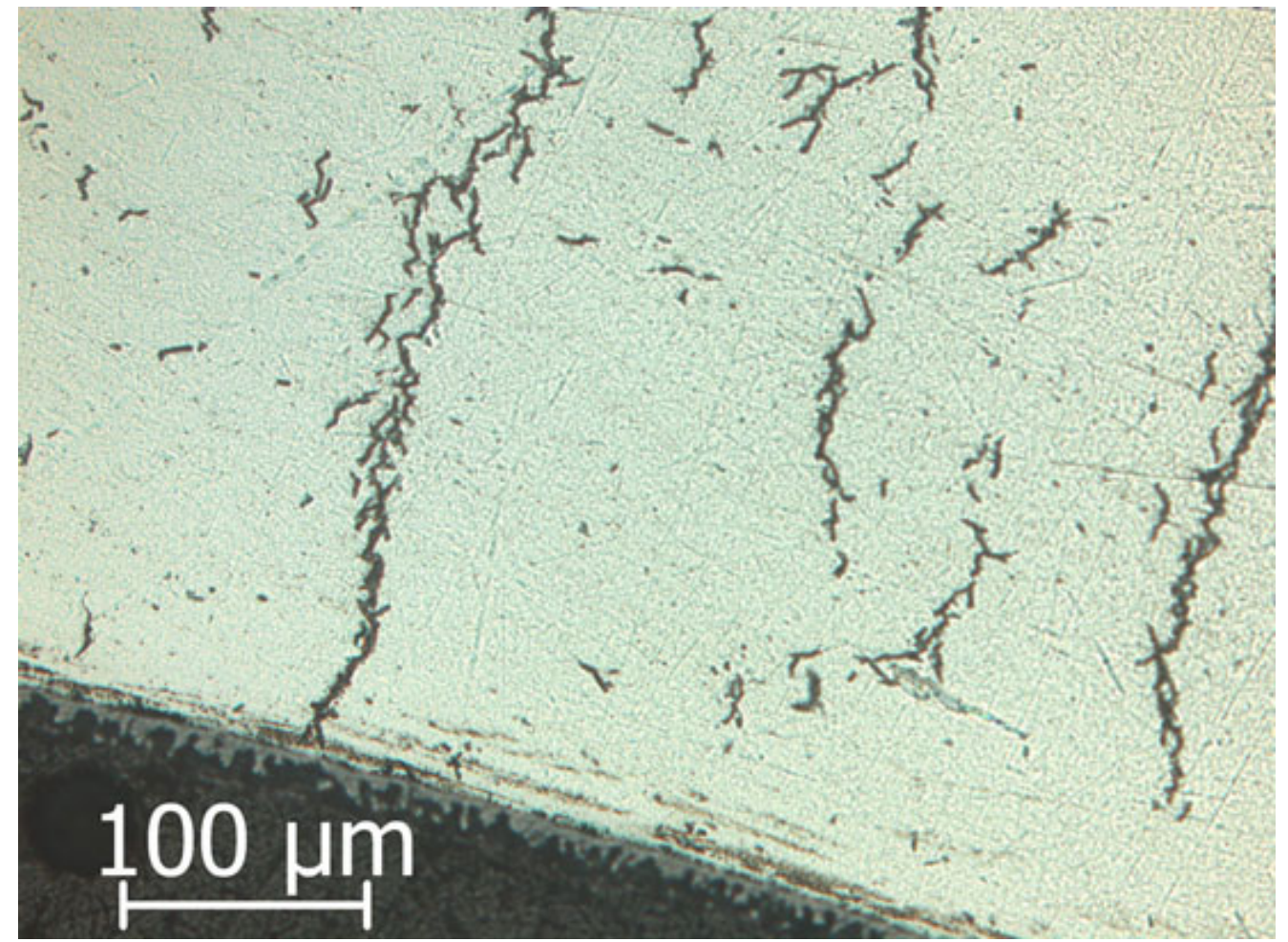

(b) $200 \mathrm{X}$

Figure 11: Longest radial hydride observed at the 12:30 orientation for the $652 \mathrm{~F} 2 \mathrm{~F}$ cross section at the reference $(0 \mathrm{~mm})$ axial location: (a) 100X and (b) 200X. 


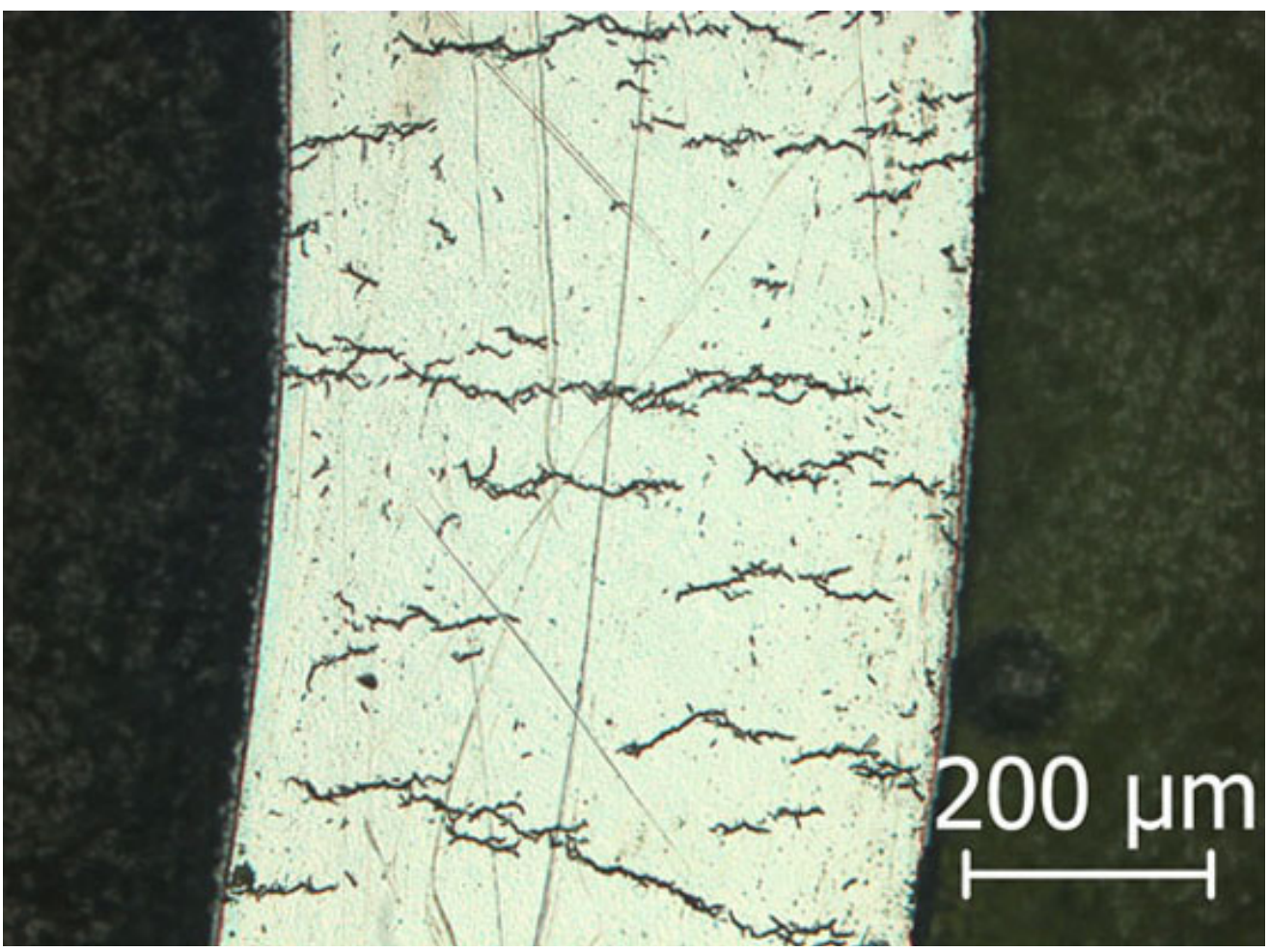

Figure 12: Longest radial hydride (bottom of figure) observed at 3:30 for the $652 \mathrm{~F} 2 \mathrm{~F}$ cross section at the $0.5-\mathrm{mm}$ axial location: (a) $100 \mathrm{X}$ and (b) $200 \mathrm{X}$

It is interesting to note that the longest radial hydride occurred at a different circumferential orientation (3:30 vs. 12:30 o'clock) than shown in Fig. 11a, and it had a different shape across the cladding wall. On the basis of 37 100X images taken at this location $(0.5 \mathrm{~mm})$, not a single radial hydride from the second set of images could be correlated with radial hydrides in the first set of images in terms of shape, length, and location across the cladding wall. These results suggest that if there were continuity of radial hydride platelets in the axial direction for the 80-wppm sample, it occurred along an axial length less than $\approx 500 \mu \mathrm{m}$. The average radial-hydride length ( $58 \pm 18 \%$ RHCF) was higher than, but comparable to, what was observed in the first set of images taken at the 0 -mm axial location.

The image shown in Fig. 12 was of low quality because of the scratches remaining on the surface even after polishing. In order to remove the scratches, additional grinding of about $0.1 \mathrm{~mm}$ was performed with fine-grit paper followed by re-polishing and re-etching. Forty three 100X images were taken of this cross section labeled as the $0.6 \mathrm{~mm}$ axial location relative to the reference surface. The longest radial hydride (96\% RHCF) occurred at the 6:30 orientation for this surface, as shown in Fig. 13a. Long radial hydrides were also observed at the 12:15 orientation. Although the hydride in Figs. 13a and $\mathrm{b}$ had about the same length as observed at the $0-\mathrm{mm}$ axial location, it had a different shape and it occurred at a different circumferential position. However, the average RHCF $(49 \pm 14 \%)$ was the same at the two locations. As with the previous comparison, none of the radial hydrides at $0.6 \mathrm{~mm}$ appeared to correlate with those observed on the $0.5-\mathrm{mm}$ surface in terms of length, shape, and location across the cladding wall. Thus, it appears that the axial extent of connected hydride platelets is $<100 \mu \mathrm{m}$ for this 80 -wppm sample. 


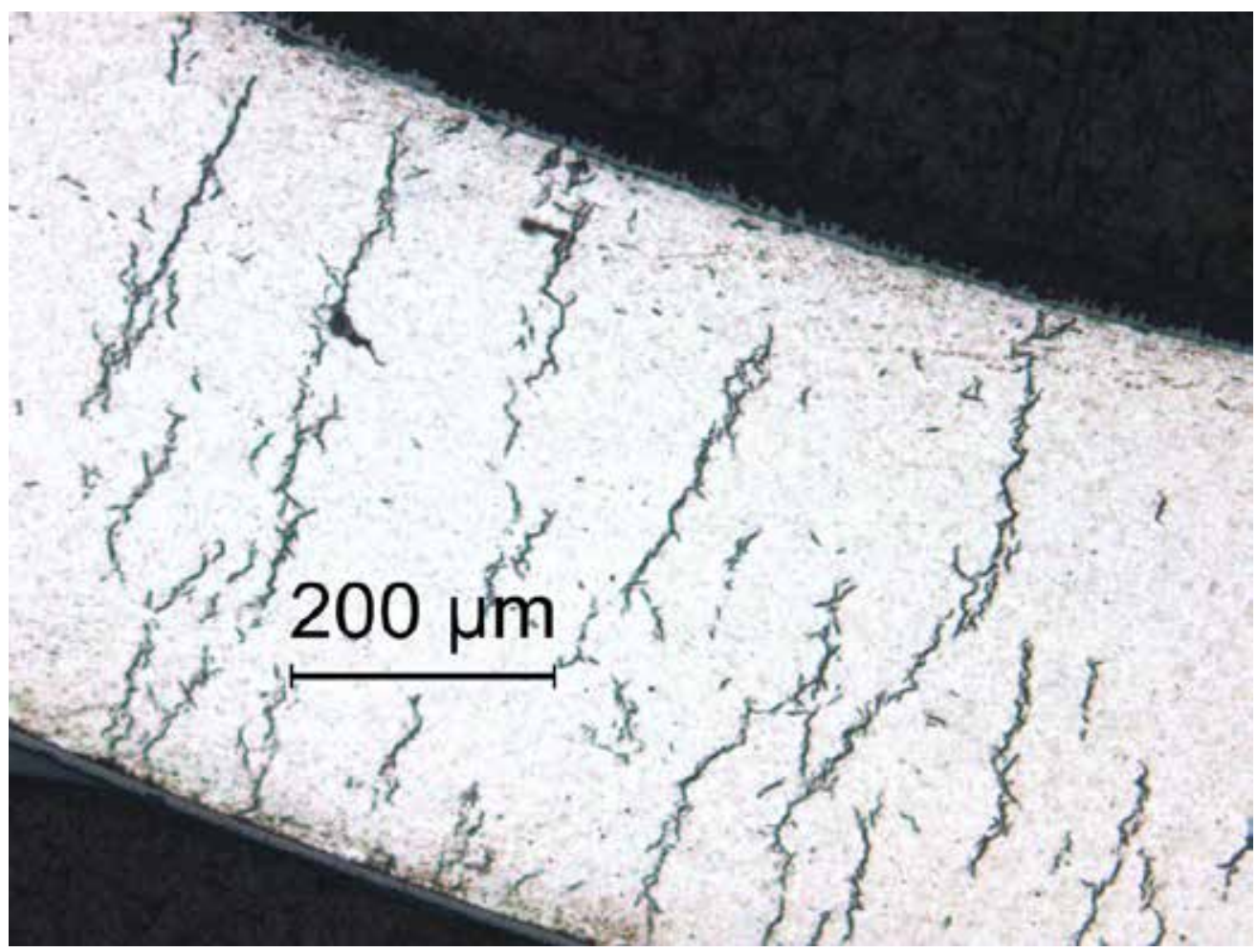

(a) $100 \mathrm{X}$

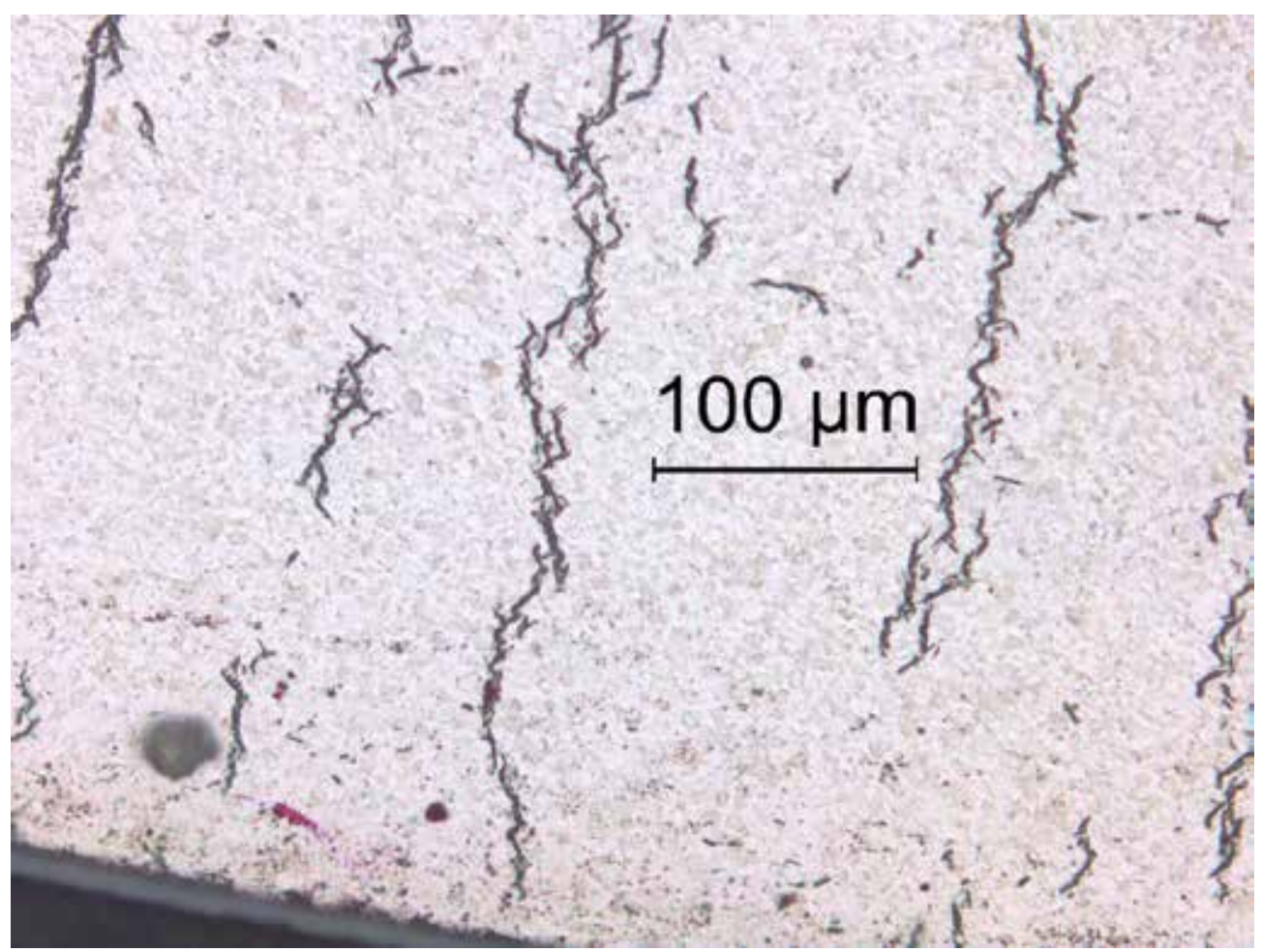

(b) $200 X$

Figure 13: Longest radial hydride observed at the 12:30 orientation for the 652F2F cross section at the $0.6 \mathrm{~mm}$ axial location: (a) $100 \mathrm{X}$ and (b) $200 \mathrm{X}$. 
The surface labeled 652FG1 was at an axial location of $3 \mathrm{~mm}$ relative to the reference location. It was at the end of a ring compressed at $120^{\circ} \mathrm{C}$. Consistent with the smooth load-displacement curve for this ring [12], no cracks were observed in the cladding metal. The thin oxide layer exhibited multiple radial cracks near the 3 and $90^{\prime}$ clock circumferential orientations where the maximum outer-surface tensile stress occurred. Figure 14 shows 100X and 200X images containing the longest radial hydride (83\% RHCF) observed at the $5: 45$ orientation. The RHCF $(37 \pm 13 \%)$ was considerably lower than what was observed for the surfaces of $652 \mathrm{~F} 2 \mathrm{~F}$ at $0 \mathrm{~mm}, 0.5 \mathrm{~mm}$ and $0.6 \mathrm{~mm}$. The mid-span of this ring, labeled $652 \mathrm{~F} 2 \mathrm{G} 2$, was at an axial location of $7 \mathrm{~mm}$ (see Fig. 10). Again, consistent with the smooth load-displacement curve for this ring, no cracking was observed. The longest radial hydride observed was only $63 \%$ of the cladding wall and the average RHCF $(30 \pm 9 \%)$ was significantly lower than what was observed at other cross sections. Figure 15 shows $100 \mathrm{X}$ and $200 \mathrm{X}$ images of the longest radial hydride at the $7 \mathrm{~mm}$ axial location.

Ring $652 \mathrm{~F} 2 \mathrm{H}$ was compressed at RT. Following the RCT, long cracks assessed as through-wall cracks were observed optically (at 1X) at both ends of the sample at the 12:00 and 6:00 o'clock orientations. The mid-span surface (652F2H1) was prepared and imaged at 100X and 200X. M ajor cracks were observed at the 12:00 (100\% of wall thickness) and 6:00 (80\% of wall thickness) o'clock orientations. These are shown in Fig. 16 at 100X magnification. The 12:00 crack (see Fig. 16a) appears to have initiated at the cladding surface at very low displacement, propagated rapidly along a continuous radial hydride through about $40 \%$ of the cladding wall, propagated more slowly along a connecting circumferential hydride to a parallel radial hydride, and propagated rapidly along the second radial hydride through the remaining $60 \%$ of the cladding wall. Although very few circumferential hydrides were observed for the six cross sections examined, a short mid-wall circumferential hydride can be seen in Fig. 16a to the right of the crack. Even though the crack obscured the hydrides along its path, it is reasonable to assume that cracking did propagate along radial-circumferential-radial hydrides. Cracks in the ductile cladding metal tend to occur at $45^{\circ}$ from the radius, which is not evident in Fig. 16a. As the RHCF is defined as the effective length across the cladding wall of continuous radial-circumferential hydrides along which a crack can propagate, the RHCF was assessed to be $100 \%$ at this location. The average RHCF for the cross section was $33+13 \%$.

At the 6:00 o'clock orientation (see Fig. 16b), the crack also appears to have initiated at the cladding surface and to have propagated rapidly along radial hydrides through about $60 \%$ of the cladding wall. The crack extension from $60 \%$ to about $80 \%$ is neither pronounced nor continuous and it likely occurred at higher displacement. The RHCF at this location was assessed to be about $60 \%$.

It is not clear which crack initiated first, but it is likely that crack initiation occurred at the 12:00 o'clock orientation before it occurred at the 6:00 o'clock orientation. There may have been some overlap in crack propagation. In general, large cracks are accompanied by large load drops ( $>25 \%$ of the load just prior to cracking). However, no such load drops were observed in the load-displacement curve for this ring (see Fig. 17). Three small load drops occurred after the load had increased to $310 \mathrm{~N}$. The minimum load (225 N) corresponds to a $27 \%$ load drop, which appears to be inconsistent with the extent of cracking observed in Fig. 16. This is an interesting case of an "implied" load drop. For the RCT conducted at $60^{\circ} \mathrm{C}$ (ring D), the load rose to $425 \mathrm{~N}$ at $1.7 \%$ corrected offset strain prior to a 39\% load drop [12]. Taking into account the differences in sample length (7.81 $\mathrm{mm}$ for ring $\mathrm{H}$ and $7.59 \mathrm{~mm}$ for ring $\mathrm{D}$ ), the ring $\mathrm{H}$ load was expected to reach about $440 \mathrm{~N}$ prior to a significant load drop. Thus, the "implied" load drop for ring $\mathrm{H}$ was about $30 \%$ prior to the observed small load drops. For this case, post-RCT metallographic images were needed to confirm embrittlement. 


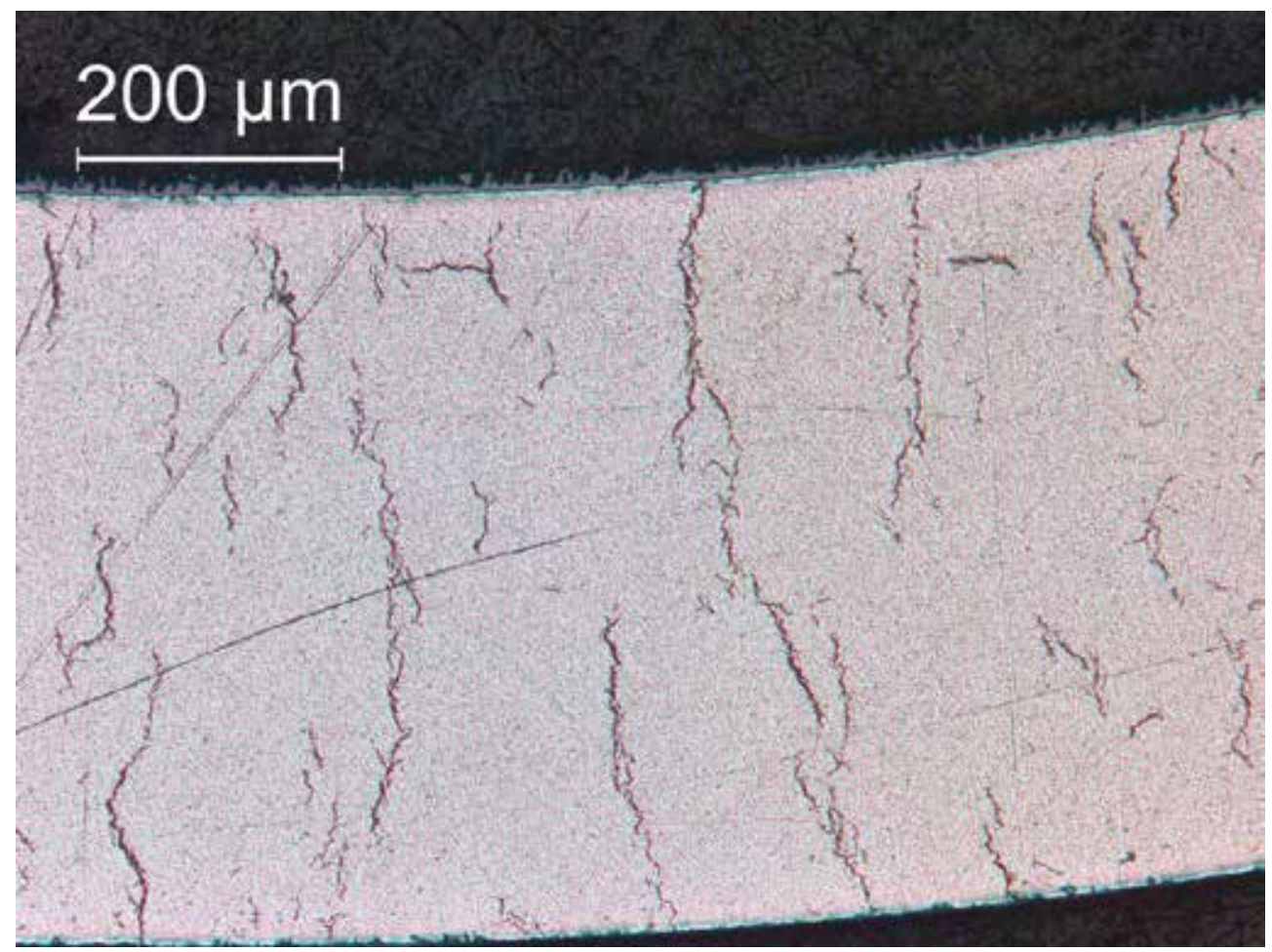

(a) $100 \mathrm{X}$

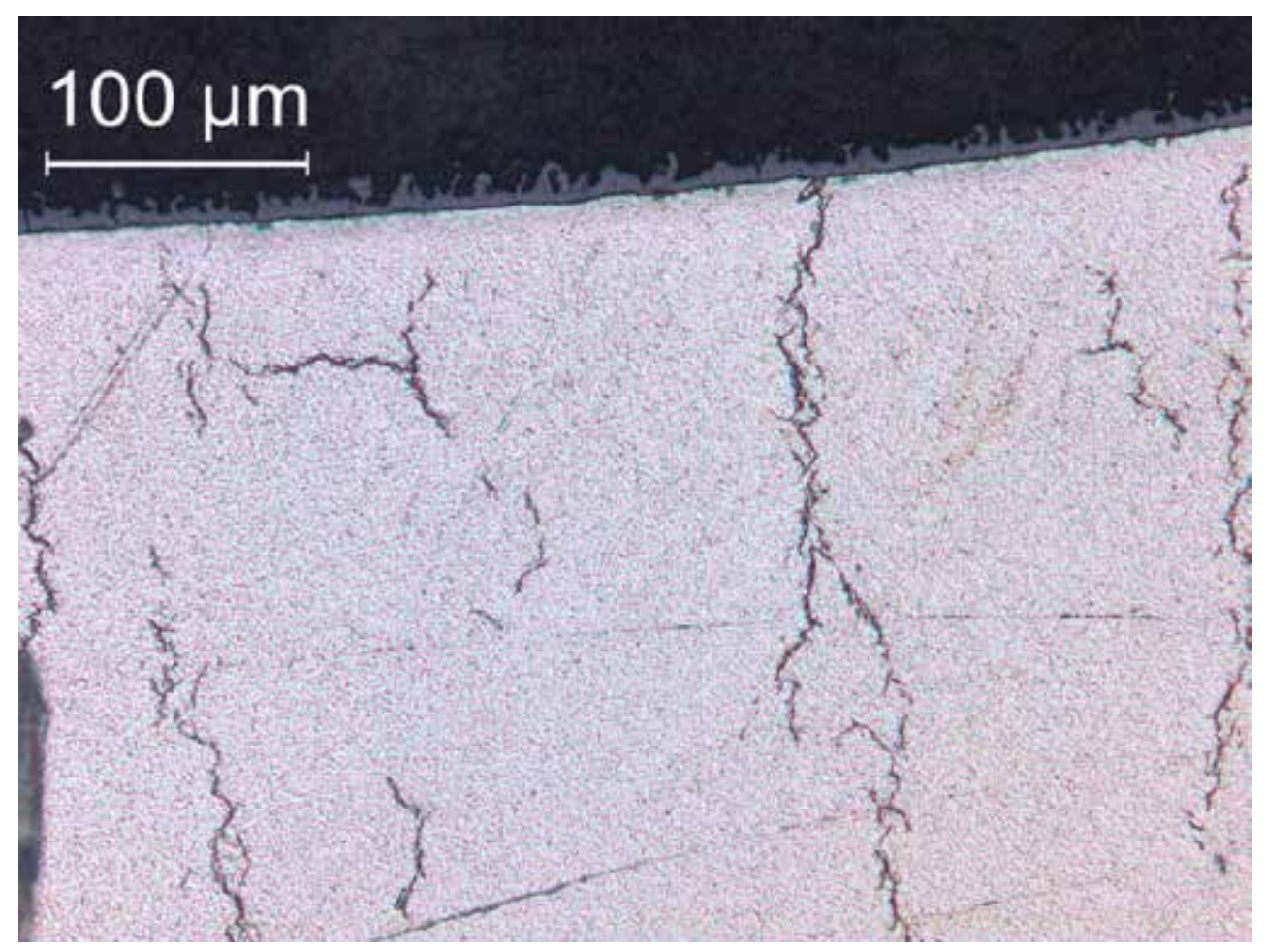

(b) $200 \mathrm{X}$

Figure 14: Longest radial hydride observed at the 5:45 orientation for the $652 \mathrm{~F} 2 \mathrm{G} 1$ cross section at the 3-mm axial location: (a) $100 \mathrm{X}$ and (b) 200X. 


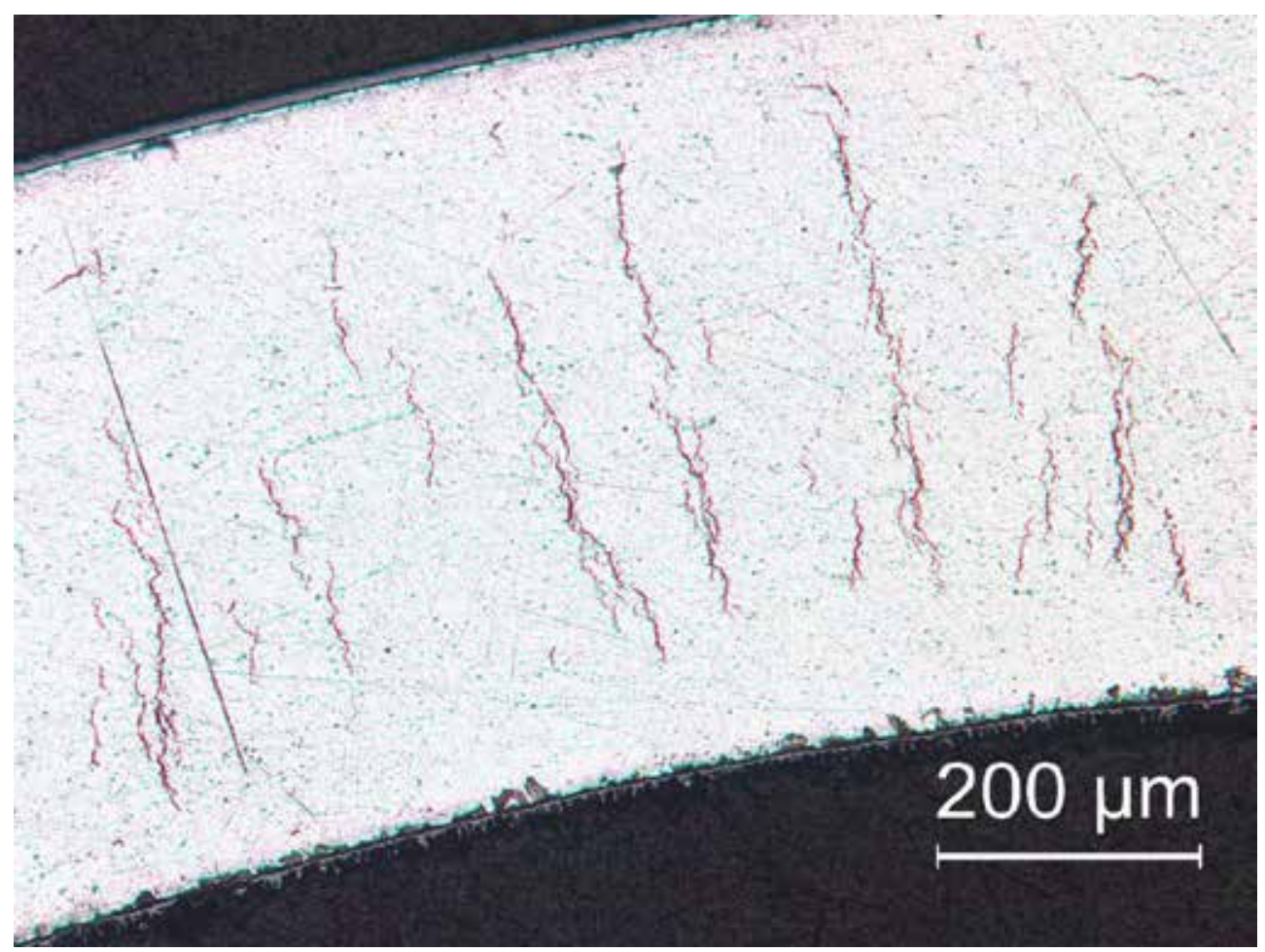

(a) $100 \mathrm{X}$

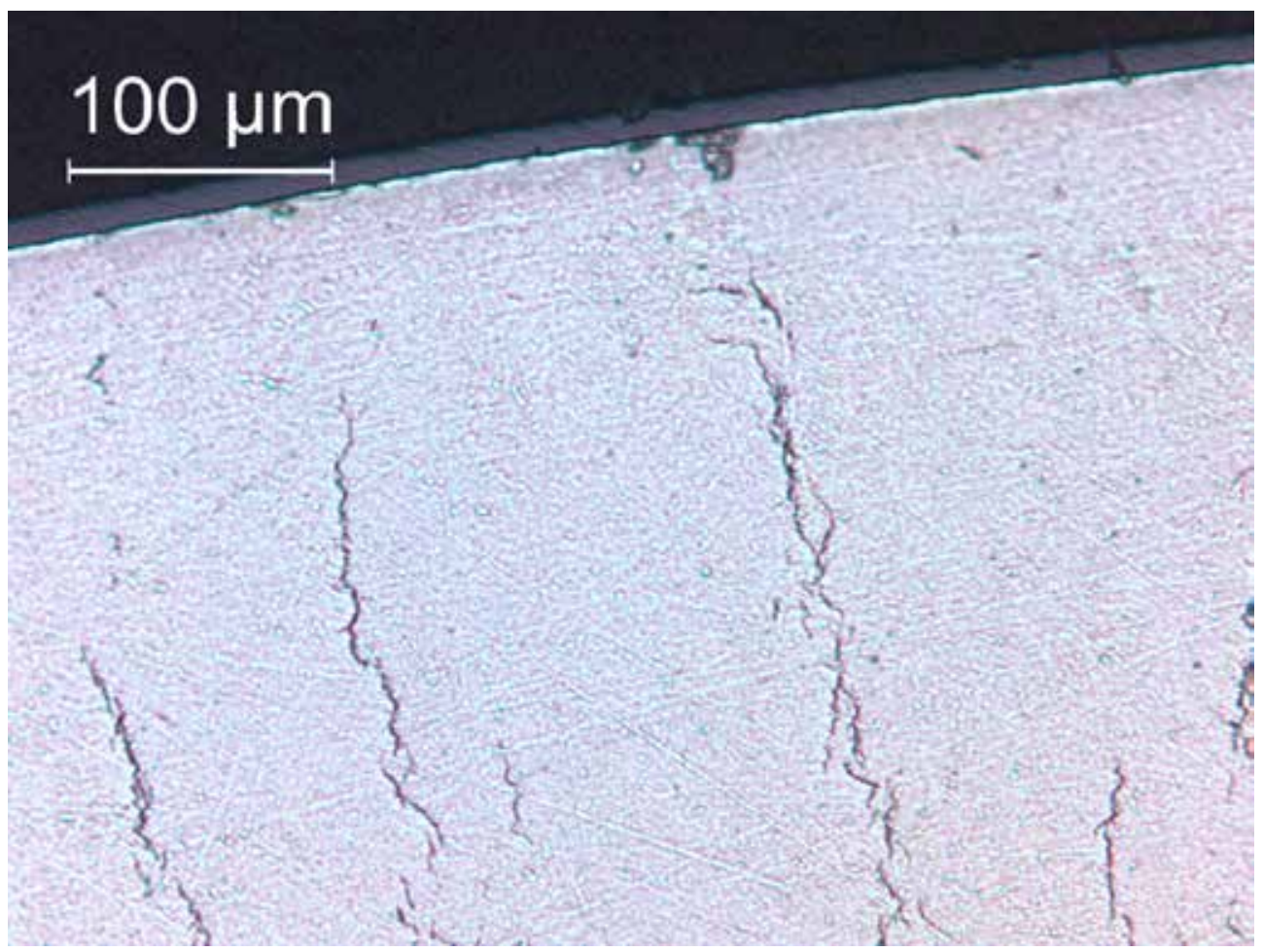

(b) $200 \mathrm{X}$

Figure 15: Longest radial hydride observed at the 11:15 orientation for the 652F2G2 cross section at the 7-mm axial location: (a) 100X and (b) 200X. 


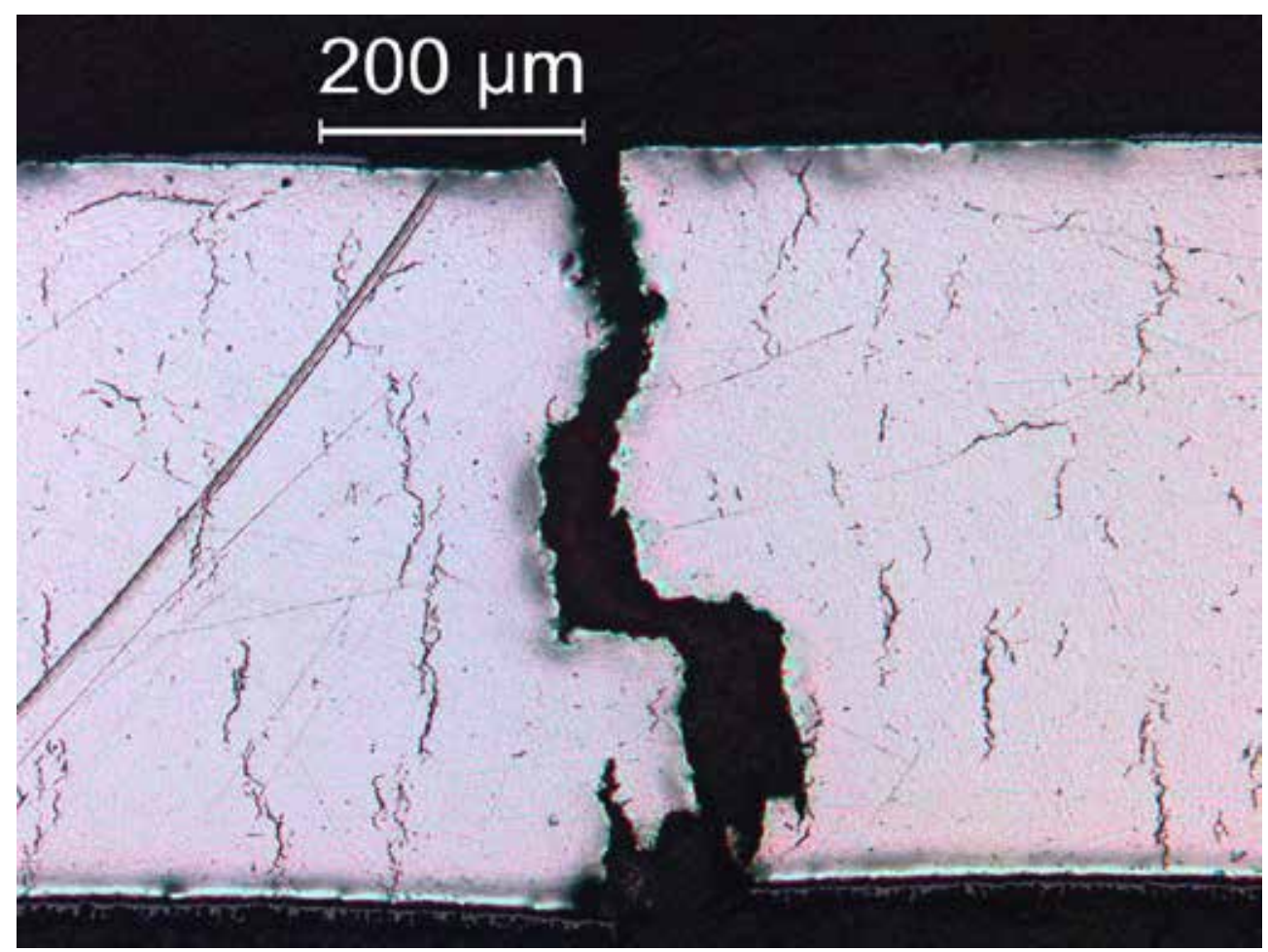

(a) 12:00 o'clock

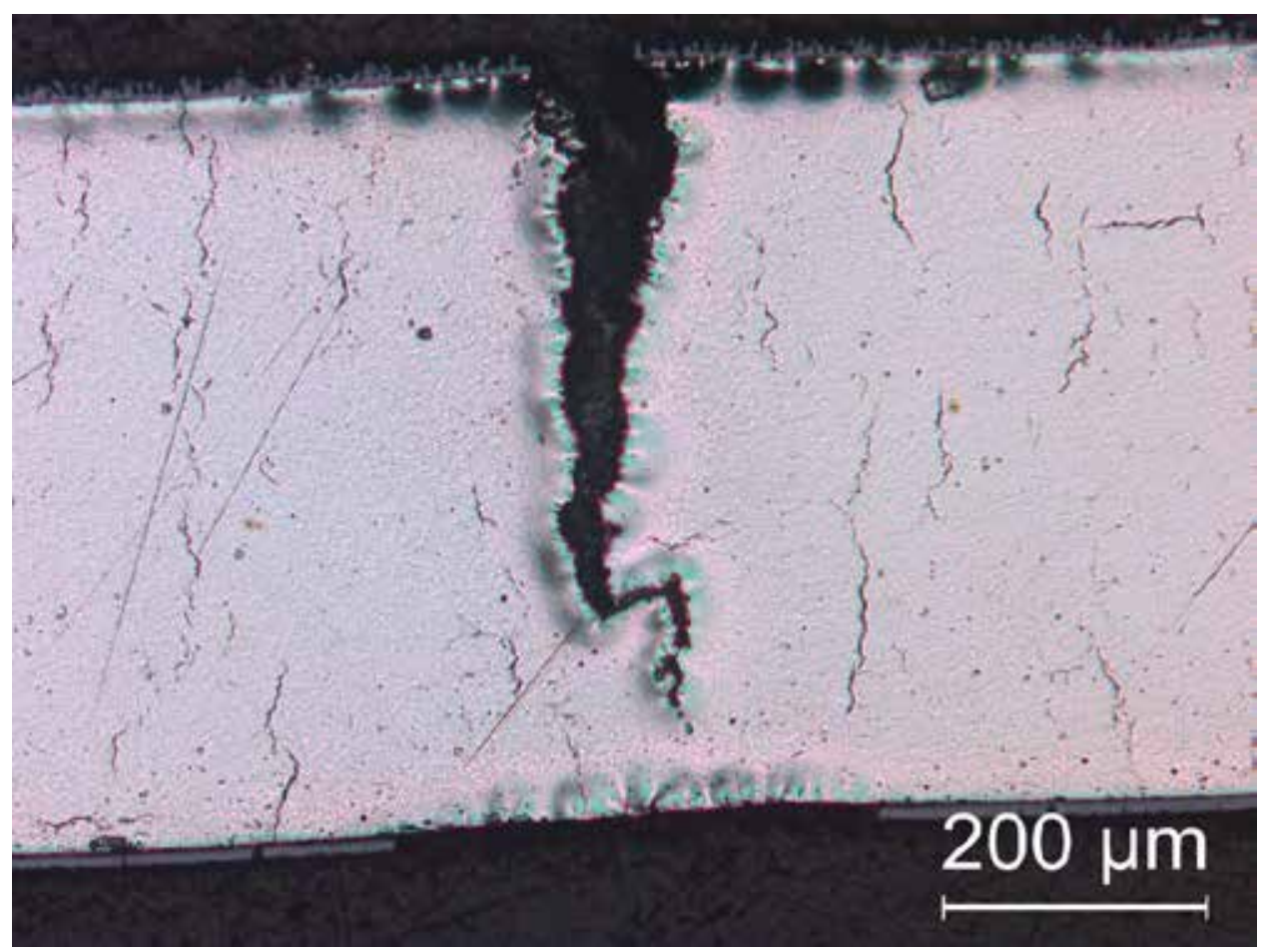

(b) 6:00 o'clock

Figure 16: Long cracks observed at the mid-span cross section of compressed ring 652F2H at the 15-mm axial location: (a) 12:00 o'clock and (b) 6:00 o'clock. 


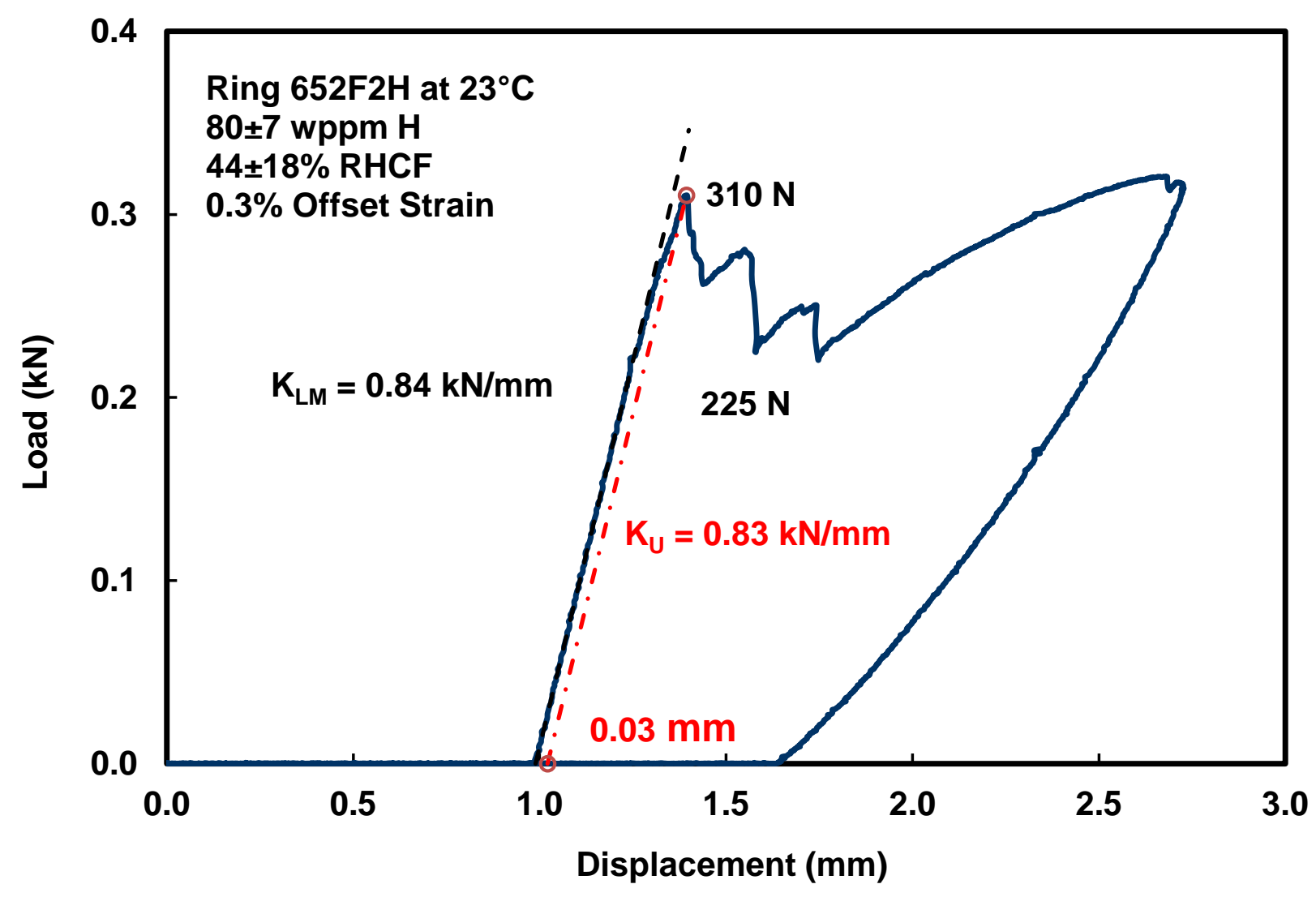

Figure 17: Load-displacement curve for the $652 \mathrm{~F} 2 \mathrm{H}$ sample tested at $23^{\circ} \mathrm{C}$.

Table 3 summarizes the RHCF results for the six HBU M5 ${ }^{\circledR}$ cross sections subjected to metallographic examination after RHT (3) and after RHT-RCT (3). The average RHCF for the six cross sections was $44 \pm 18 \%$. The results confirm the hypothesis that radial hydrides have low continuity in the axial direction for $\mathrm{HBU} \mathrm{M}^{\circledR}{ }^{\circledR}$ cladding with $\leq 80$ wppm hydrogen. However, the extensive cracking observed (1X) at both ends of ring $652 \mathrm{~F} 2 \mathrm{H}$ and at the sample mid-span (100X and 200X) suggest that radial hydrides are close enough in the axial direction to promote continuous radial cracks along the length of the sample. Taking into account all results for $\mathrm{HBU} \mathrm{M} 5{ }^{\circledR}$ it appears that there is a minimum hydrogen content in the range of 60-70 wppm below which radial hydride embrittlement will not occur regardless of the peak RHT stress.

Table 3 Results of metallographic examination for six cross sections of HBU M5 ${ }^{\circledR}$ rodlet $652 \mathrm{~F} 2$.

\begin{tabular}{c|c|c|c|c}
$\begin{array}{c}\text { Sample } \\
\text { ID }\end{array}$ & $\begin{array}{c}\text { Axial } \\
\text { Location, mm }\end{array}$ & $\begin{array}{c}\text { Average } \\
\text { RHCF, \% }\end{array}$ & $\begin{array}{c}\text { Peak RHCF, } \\
\%\end{array}$ & $\begin{array}{c}\text { Circumferential } \\
\text { Location of } \\
\text { Peak RHCF }\end{array}$ \\
\hline $652 \mathrm{~F} 2 \mathrm{~F}$ & 0 & $49 \pm 14$ & 95 & $12: 30$ \\
$652 \mathrm{~F} 2 \mathrm{~F}$ & 0.5 & $54 \pm 18$ & 96 & $3: 30$ \\
$652 \mathrm{~F} 2 \mathrm{~F}$ & 0.6 & $49 \pm 14$ & 96 & $6: 30$ \\
$652 \mathrm{~F} 2 \mathrm{G} 1$ & 3 & $37 \pm 13$ & 83 & $5: 45$ \\
$652 \mathrm{~F} 2 \mathrm{G} 2$ & 7 & $30 \pm 9$ & 63 & $11: 15$ \\
$652 \mathrm{~F} 2 \mathrm{H} 1$ & 15 & $33 \pm 13$ & 100 & $12: 00$ \\
\hline
\end{tabular}




\subsection{AXIAL CONTINUITY OF RADIAL HYDRIDES IN HBU ZIRLO ${ }^{\text {TM }}$}

HBU ZIRLO ${ }^{\mathrm{TM}}$ rodlet $646 \mathrm{D}$ contained $387 \pm 72 \mathrm{wppm}$ hydrogen and was subjected to peak RHT conditions of $350^{\circ} \mathrm{C}$ and $87 \mathrm{MPa}$ for 24 hours prior to slow cooling to $130^{\circ} \mathrm{C}$. On the basis of RCT results, the DTT was assessed to be very low $\left(28^{\circ} \mathrm{C}\right)$, consistent with the low RHCF $(20+11 \%)$ based on one pre-RCT cross section. In the current work, the RHCF was determined for four post-RCT cross sections and crack number, length, and morphology were examined to determine the degree of axial continuity of radial and circumferential hydrides. Figure 18 shows the sectioning diagram indicating the axial locations for the post-RCT cross sections subjected to metallographic examination. Ring 646D4, which was compressed at $60^{\circ} \mathrm{C}$ and exhibited cracking based on the load-displacement curve [12], was examined at the mid-span (4A) and at the right end (4B). Ring 646D7, which was compressed at $90^{\circ} \mathrm{C}$ and exhibited no cracking based on the load-displacement curve [12], was examined at the left end (7A) and at the mid-span (7B).

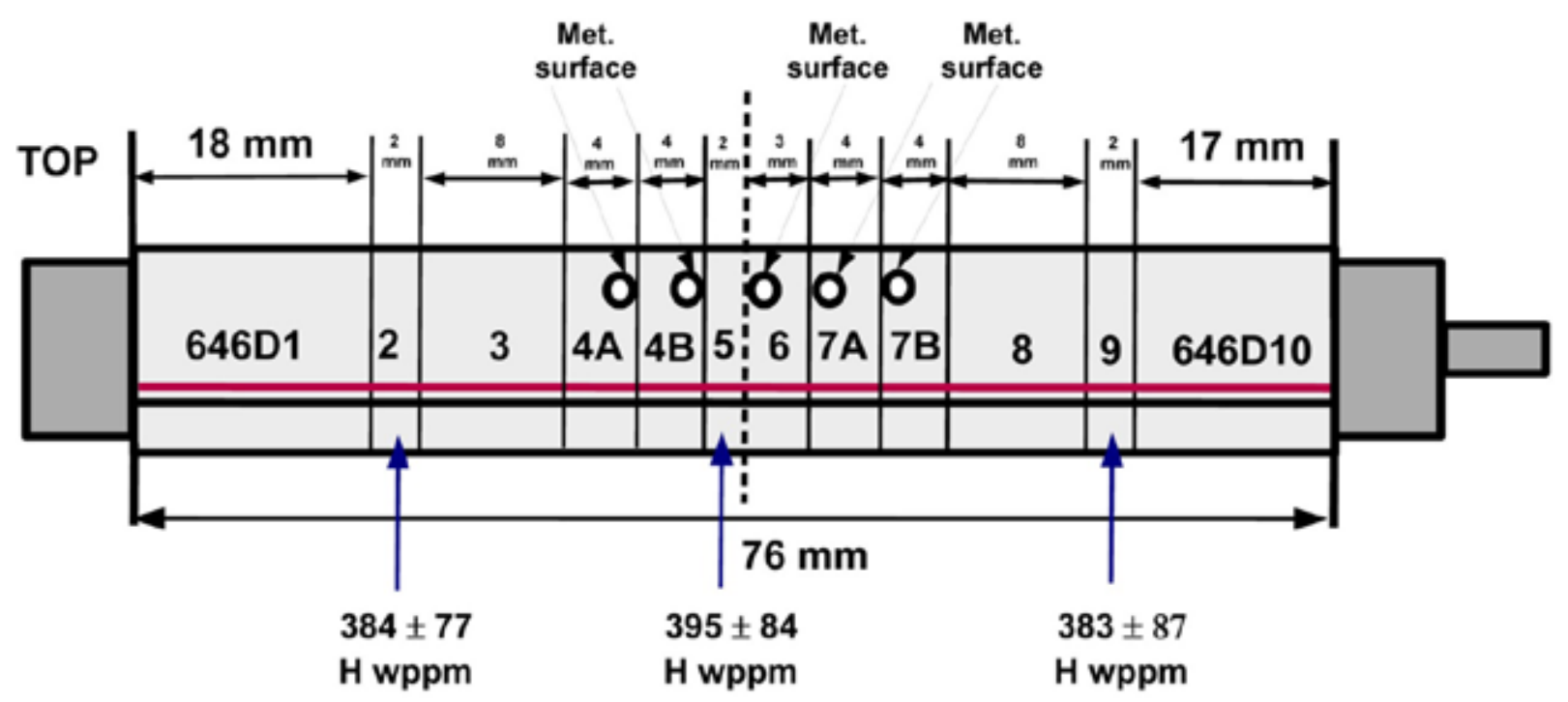

Figure 18: Sectioning diagram for the HBU ZIRLO $^{\text {TM }}$ rodlet $646 \mathrm{D}$ following RHT and RCT.

M etallographic results for reference ring $646 \mathrm{D} 6$ at $0 \mathrm{~mm}$ axial location were presented in Ref. 12 . These results are repeated below for the convenience of the reader.

Figure 19 shows 100X and 200X images of the longest radial hydride (52\% RHCF) located at the 12:45 orientation of the $646 \mathrm{D} 6$ cross section. As indicated above, the RHCF was $20 \pm 11 \%$ for this cross section. Figure 20 shows the major crack that initiated at the outer surface of the 3:00 o'clock orientation of cross section 646D4A. The crack propagated radially though the hydride rim, circumferentially a short distance, along a $45^{\circ}$ angle through the matrix metal, circumferentially along a hydride, and radially along a radial hydride into about $60 \%$ of the cladding wall. Beyond that point there was discontinuity in the crack followed by cracking along radial-circumferential hydrides. Previous experience indicates cracking occurred along circumferential hydrides prior to cracking within the matrix metal between the cracked hydrides. On the basis of the crack width at the outer surface, crack initiation occurred after significant offset strain. The maximum RHCF (50\%) and a minor secondary crack occurred at a location just to the left of the major crack at what we will call the 2:45 o'clock orientation. Figure 21 shows a micrograph from a 200X image for this location. It is interesting to note that the secondary crack did not propagate through the full $50 \%$ of the cladding wall because of the tortuous pathway though the "nest" of short radial-circumferential hydrides. The average RHCF for cross section 646D4A was $17 \pm 11 \%$ 


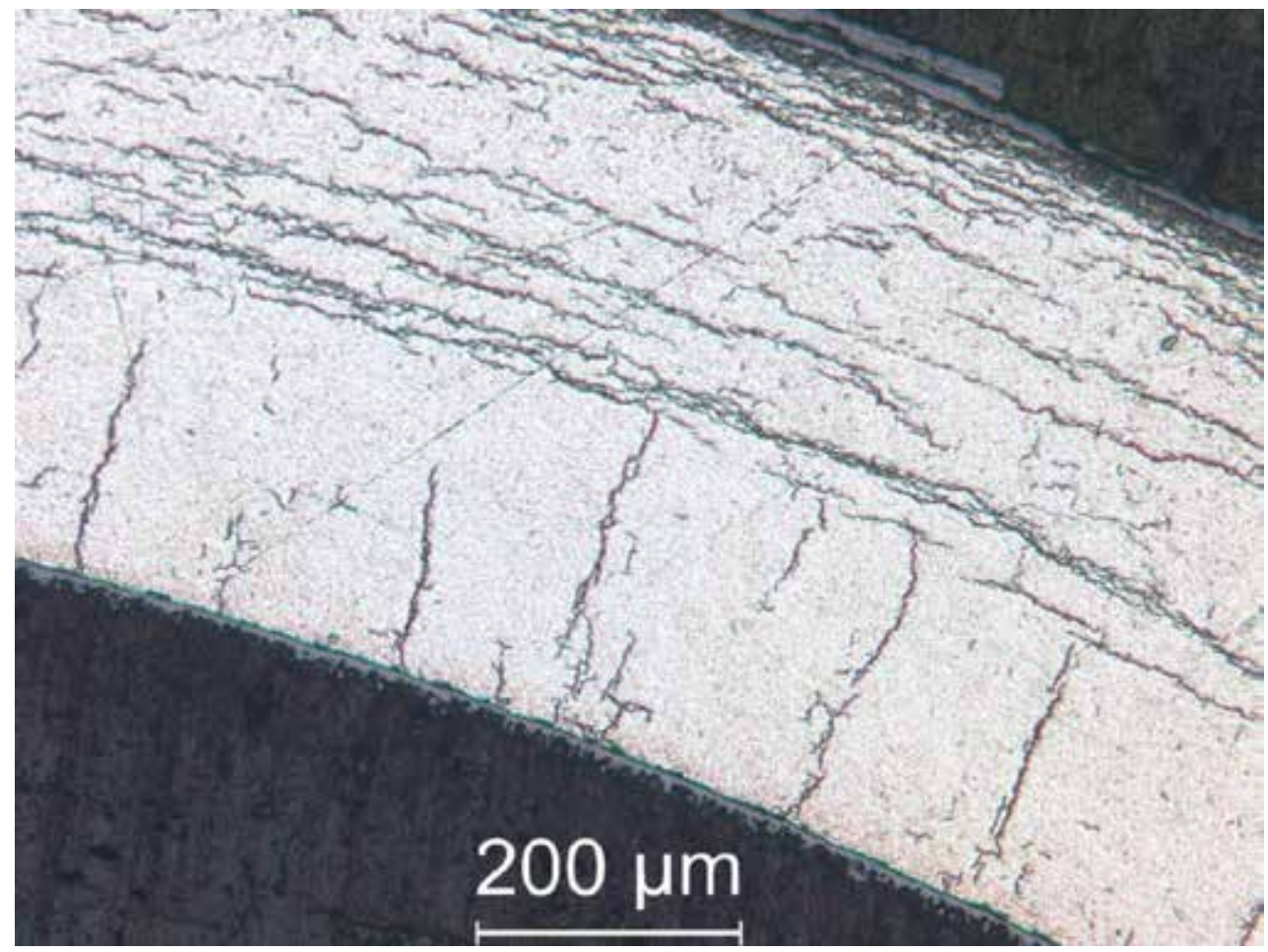

(a) $100 \mathrm{X}$

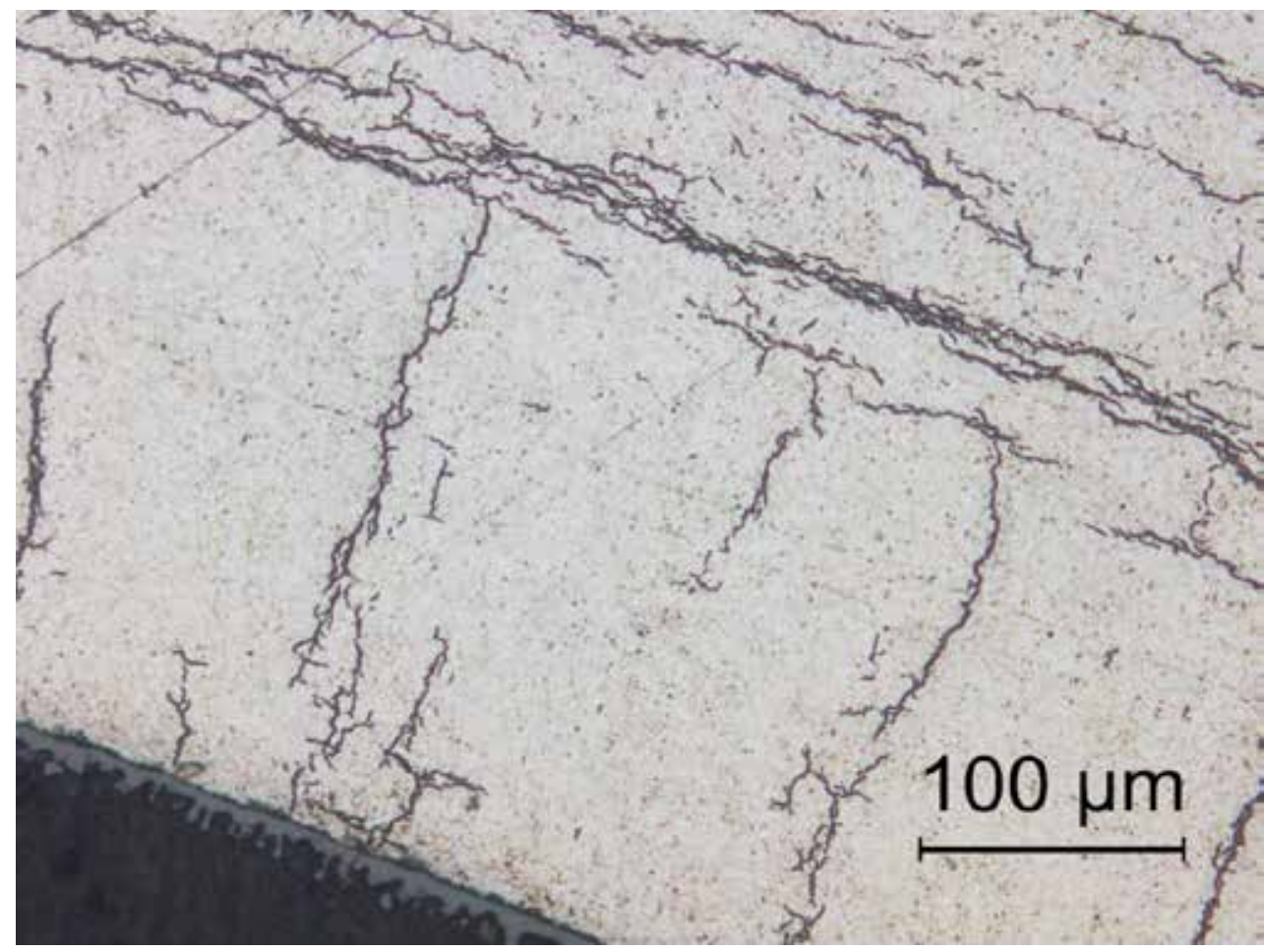

(b) $200 \mathrm{X}$

Figure 19: Images of the longest radial hydride observed for the 646D6 cross section $(0 \mathrm{~mm}$ ), which occurred at the 12:45 o'clock orientation: (a) 100X and (b) 200X. 


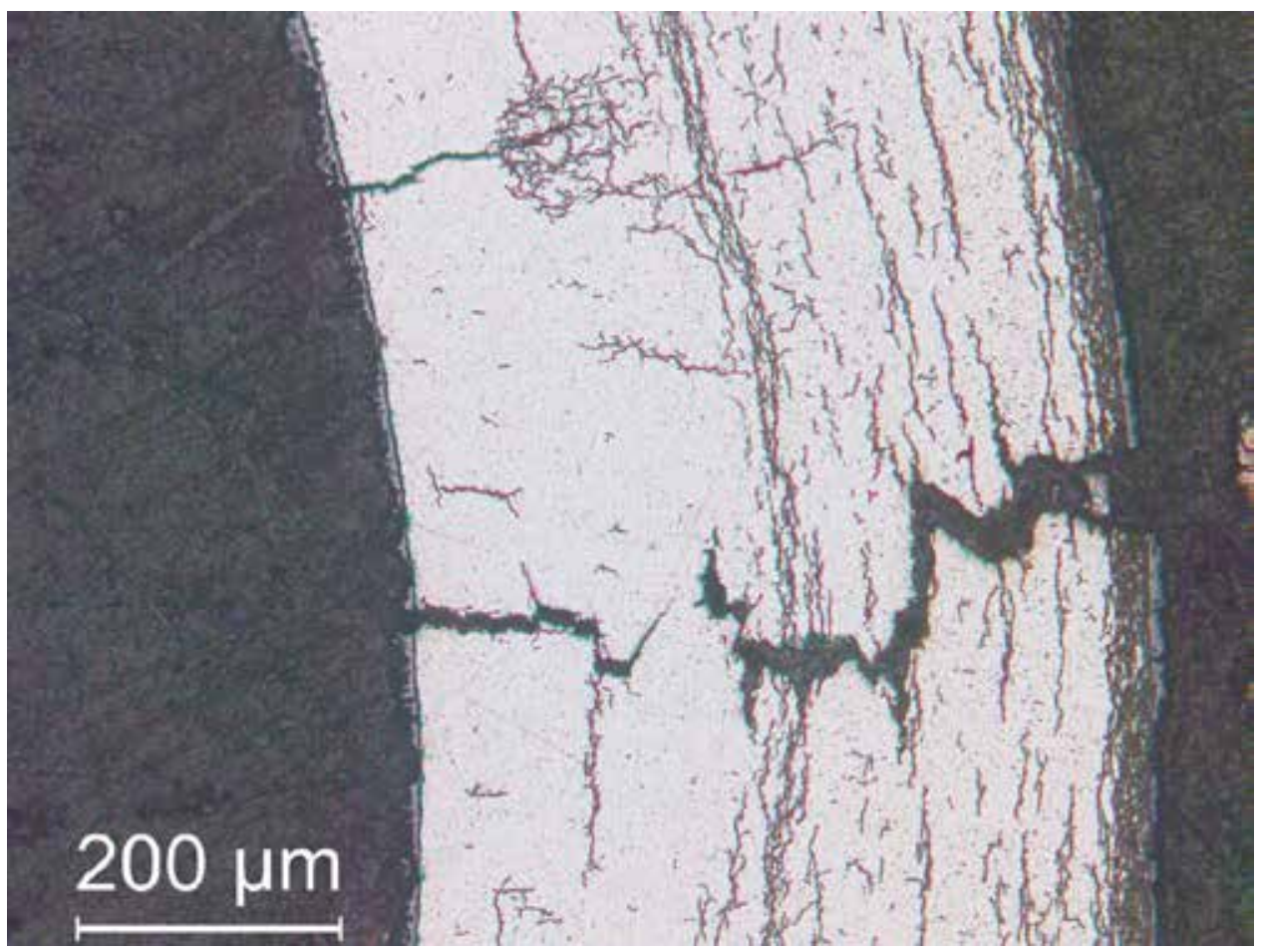

(a) $100 \mathrm{X}$

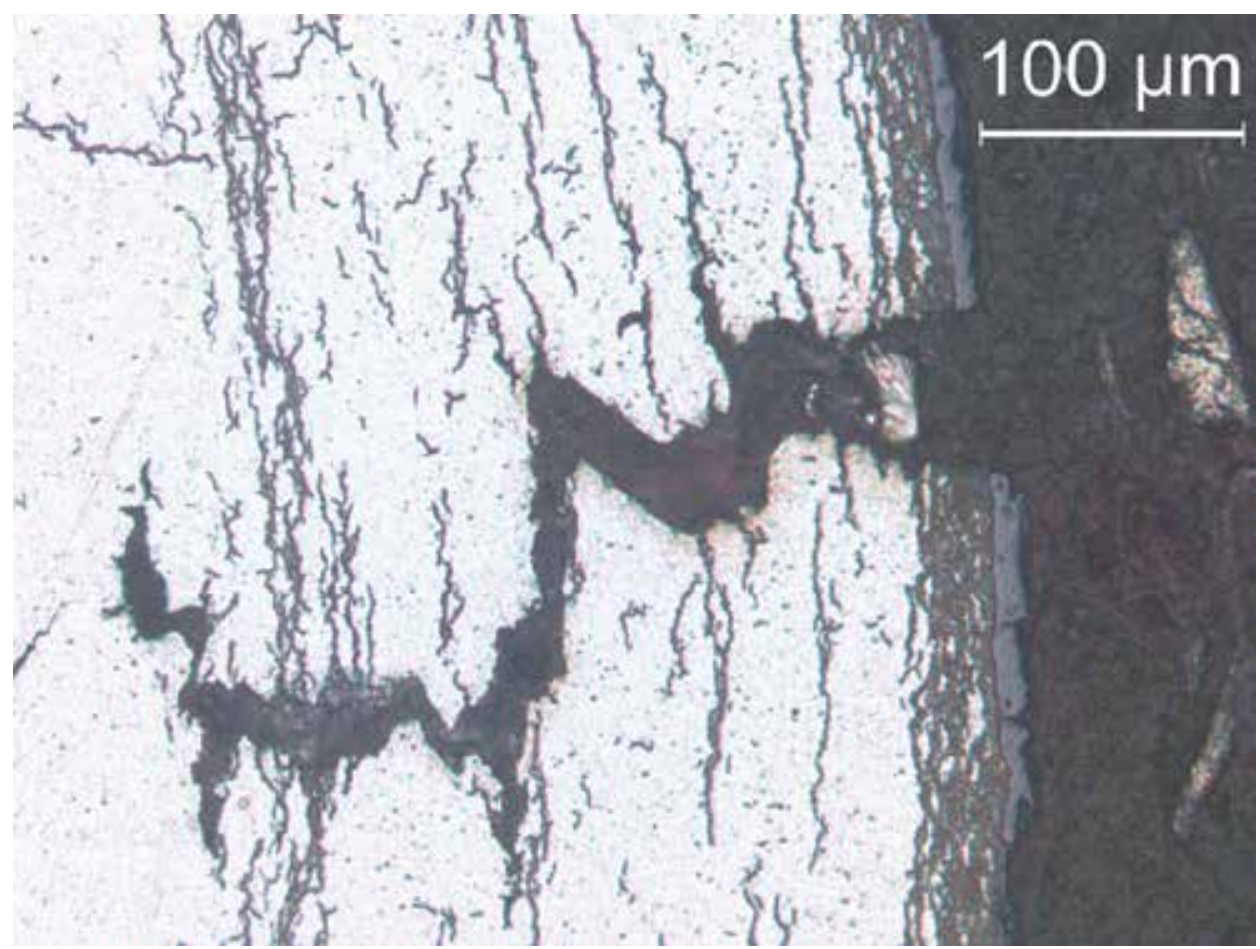

(b) $200 \mathrm{X}$

Figure 20: Major crack observed at the 3:00 o'clock orientation of the 646D4A cross section at the $-6-\mathrm{mm}$ axial location: (a) 100X and (b) $200 \mathrm{X}$. 


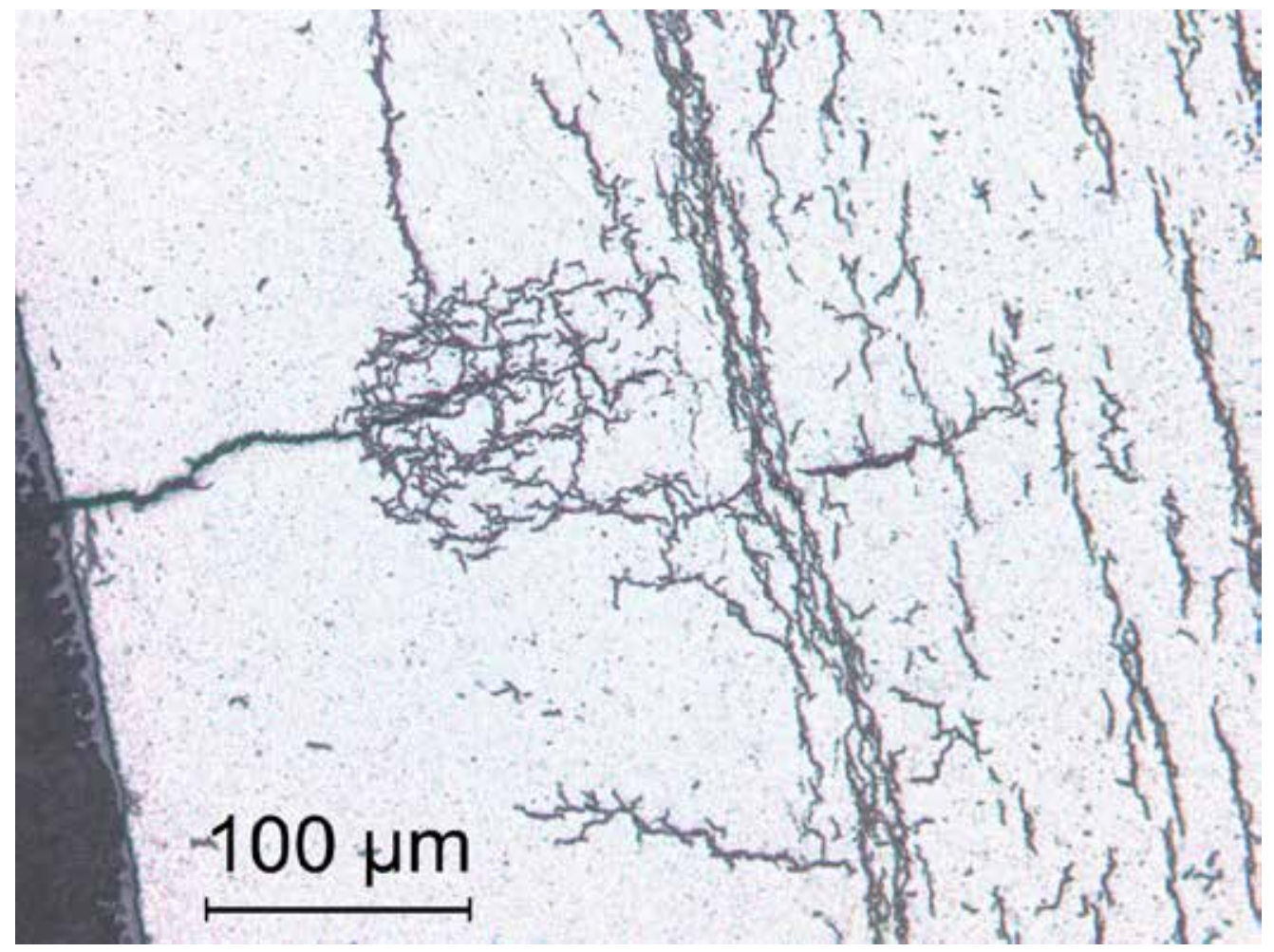

Figure 21: Image of the longest radial hydride for the 646D4A cross section $(-6 \mathrm{~mm})$, which occurred at the 2:45 o'clock orientation.

Cross section 646D4B exhibited a similar cracking pattern at the 3:00 o'clock orientation. As shown in Fig. 22 , there was one major crack extending continuously from the outer surface through about $60 \%$ of the cladding wall and discontinuously through the remaining $40 \%$ of the cladding wall. In addition, minor cracks were observed to occur at the outer surface just above (about 2:45 o'clock orientation) the major crack and at the inner surface just below the major crack. Similar to what was observed for 646D4A, most of the crack propagation occurred after significant offset strain. The results for 646D4A and 646D4B indicate that the major crack extended along the length of the sample, crack initiation occurred at the cladding outer surface at the 3:00 o'clock orientation, and radial hydrides contributed to crack propagation. In terms of the RHCF, the average value for the 646D4B cross section at an axial location of $-2 \mathrm{~mm}$ was $14 \pm 6 \%$. The maximum RHCF (30\%), which is shown in Fig. 23 , occurred at the 8:45 o'clock orientation.

For the 646D7A ( $3 \mathrm{~mm}$ ) and 646D7B (7 mm) cross sections, the average RHCF values were $15 \pm 8 \%$ and $10 \pm 6 \%$, respectively. The maximum RHCF was $35 \%$ at the $8: 45$ o'clock orientation for 646D7A (see Fig. 24 ) and $26 \%$ at the 2:00 o'clock orientation for 646D7B (see Fig. 25). 


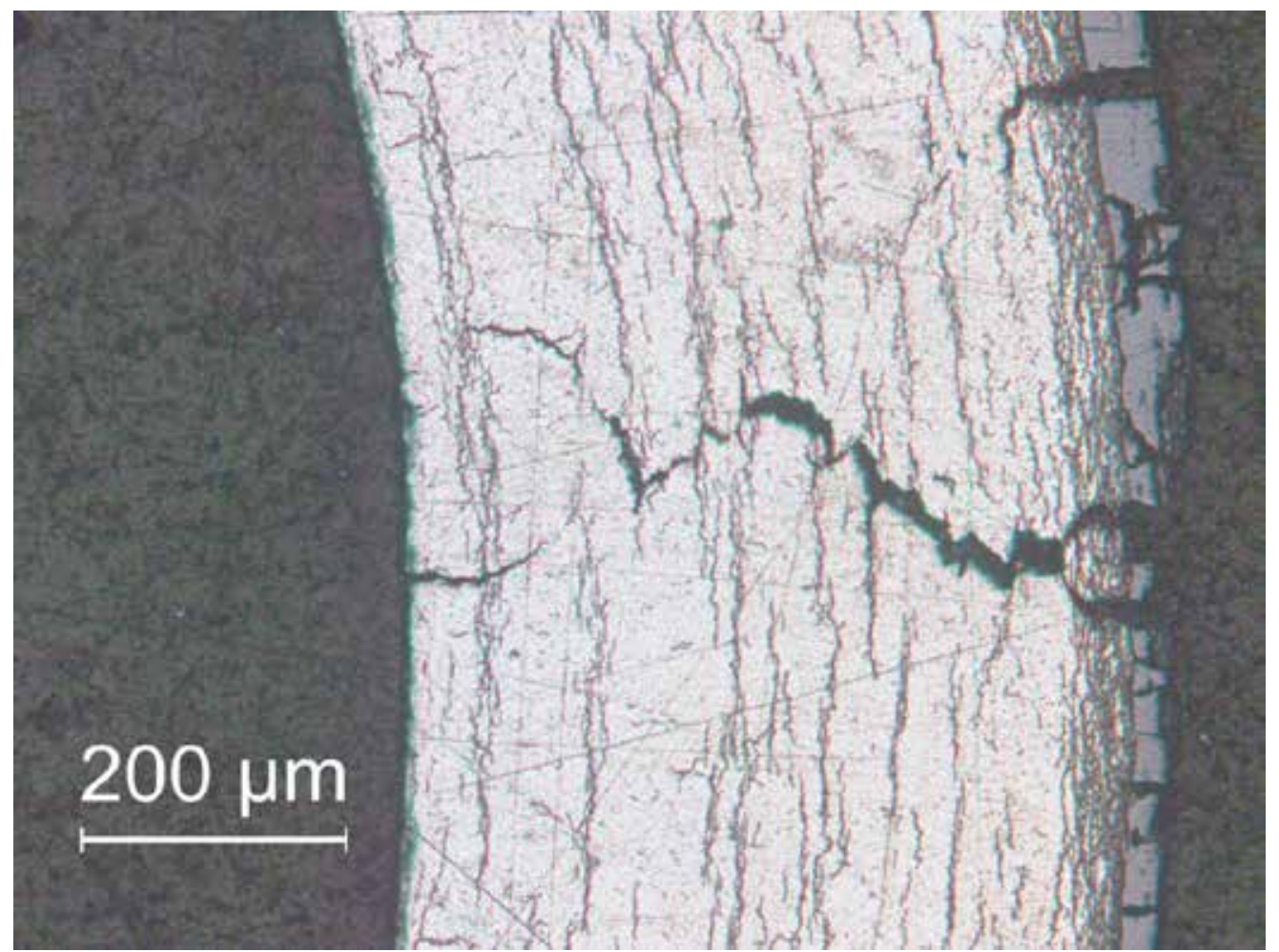

(a) $100 \mathrm{X}$

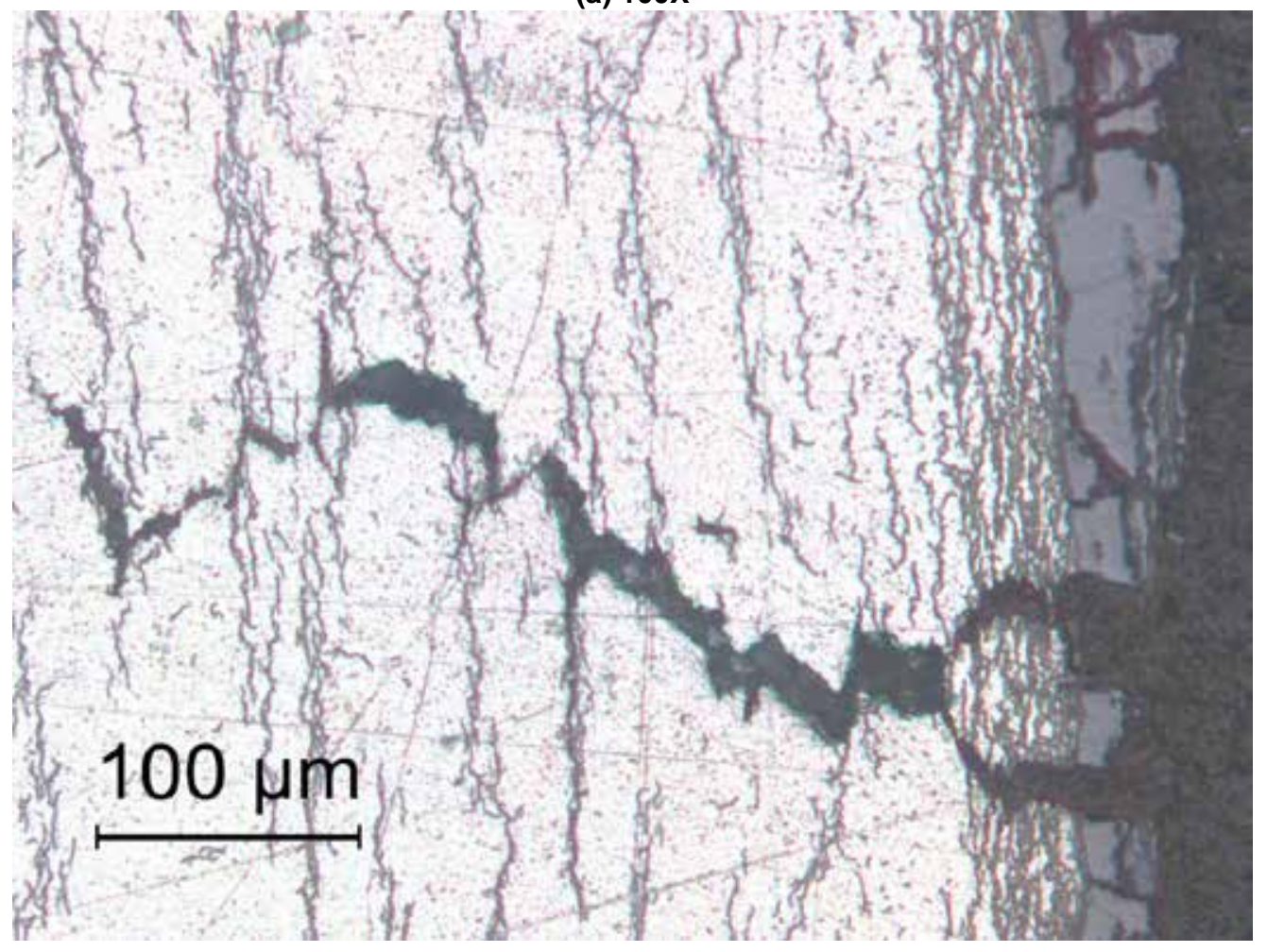

(b) $200 \mathrm{X}$

Figure 22: Major crack observed at the 3:00 o'clock orientation of the 646D4B cross section at the $-6-\mathrm{mm}$ axial location: (a) $100 \mathrm{X}$ and (b) $200 \mathrm{X}$. 


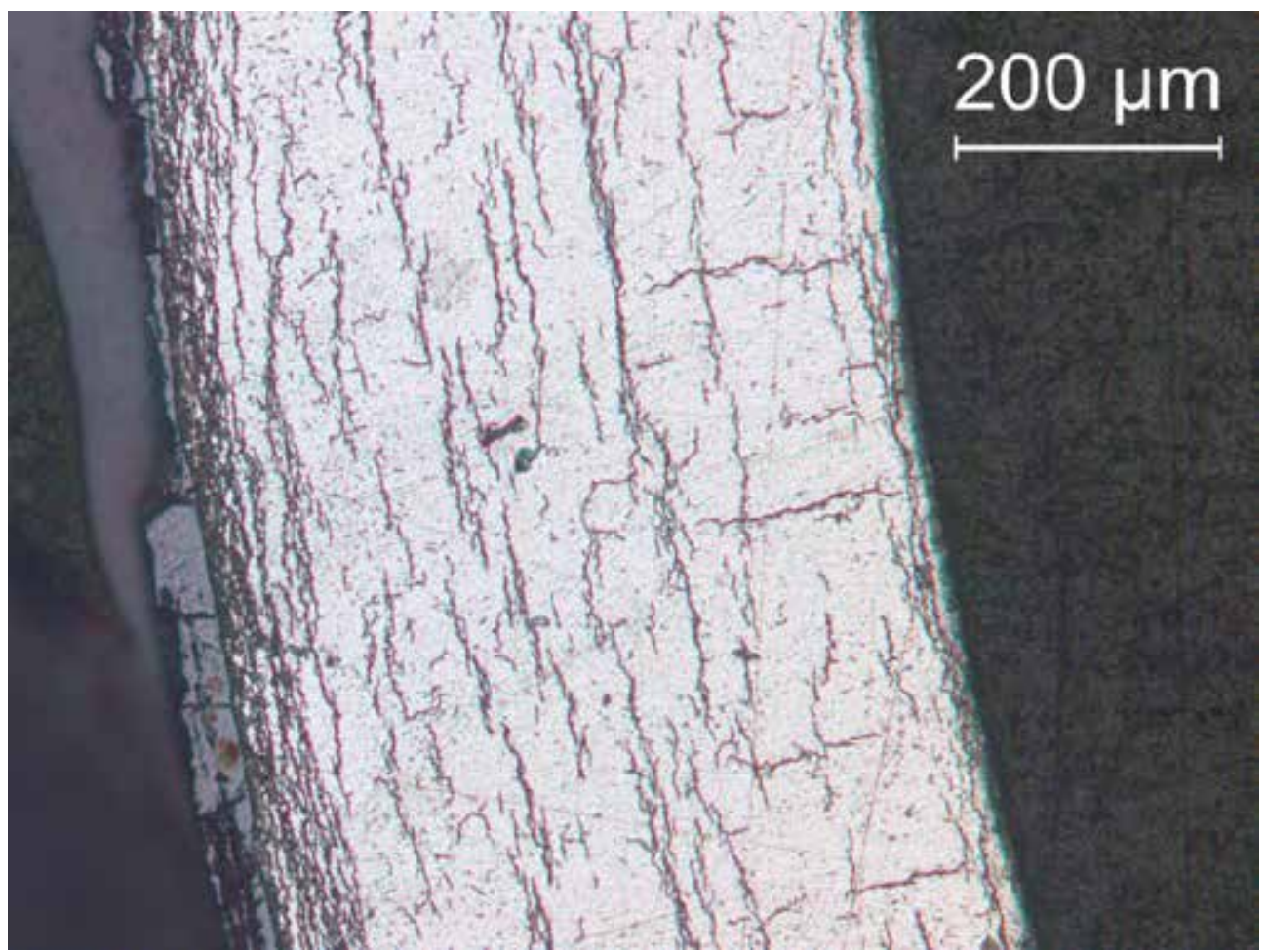

(a) $100 \mathrm{X}$

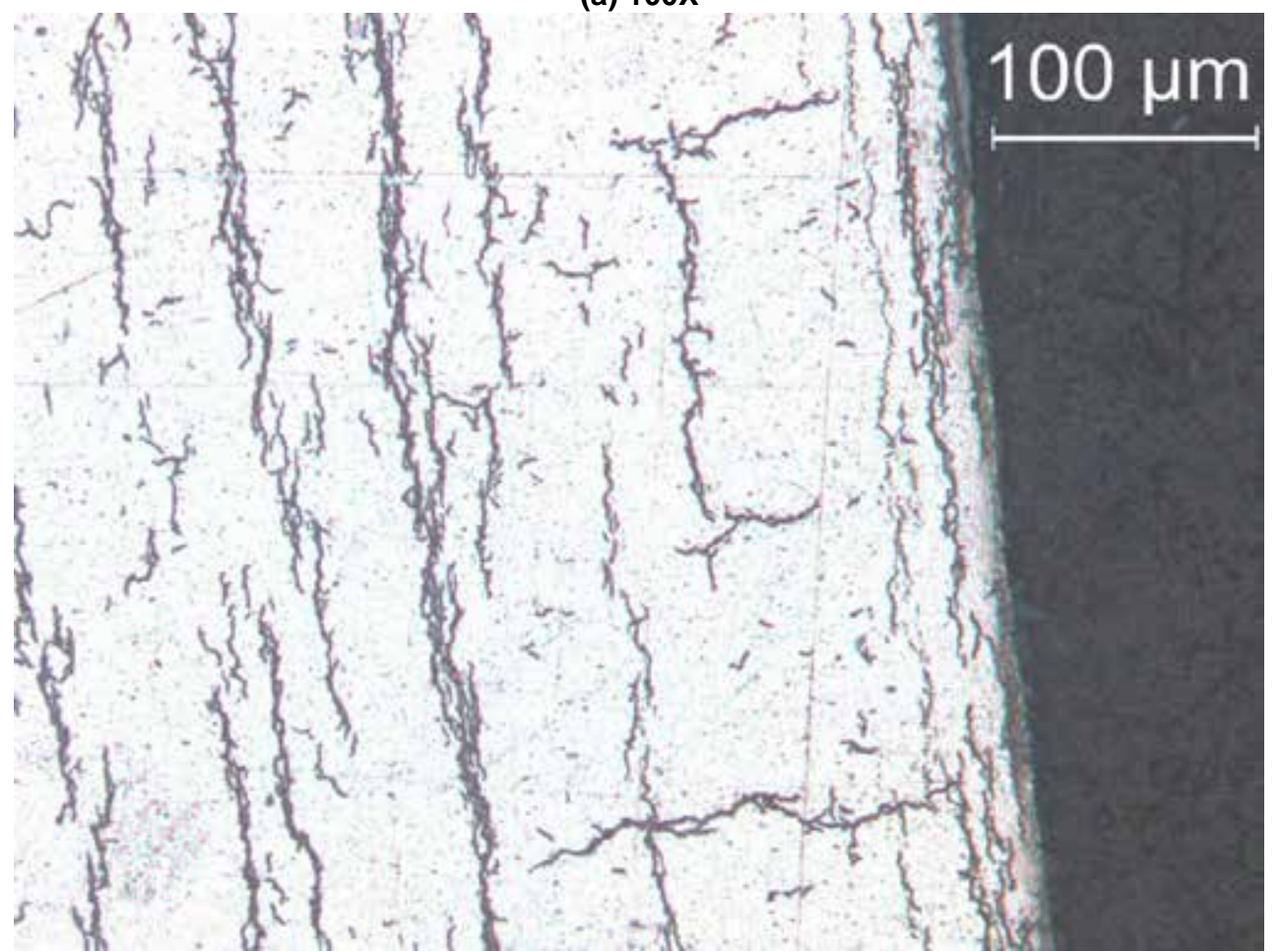

(b) $200 X$

Figure 23: Images of the longest radial hydride for the 646D4B cross section $(-2 \mathrm{~mm})$, which occurred at the 8:45 o'clock orientation: (a) 100X and (B) 200X. 


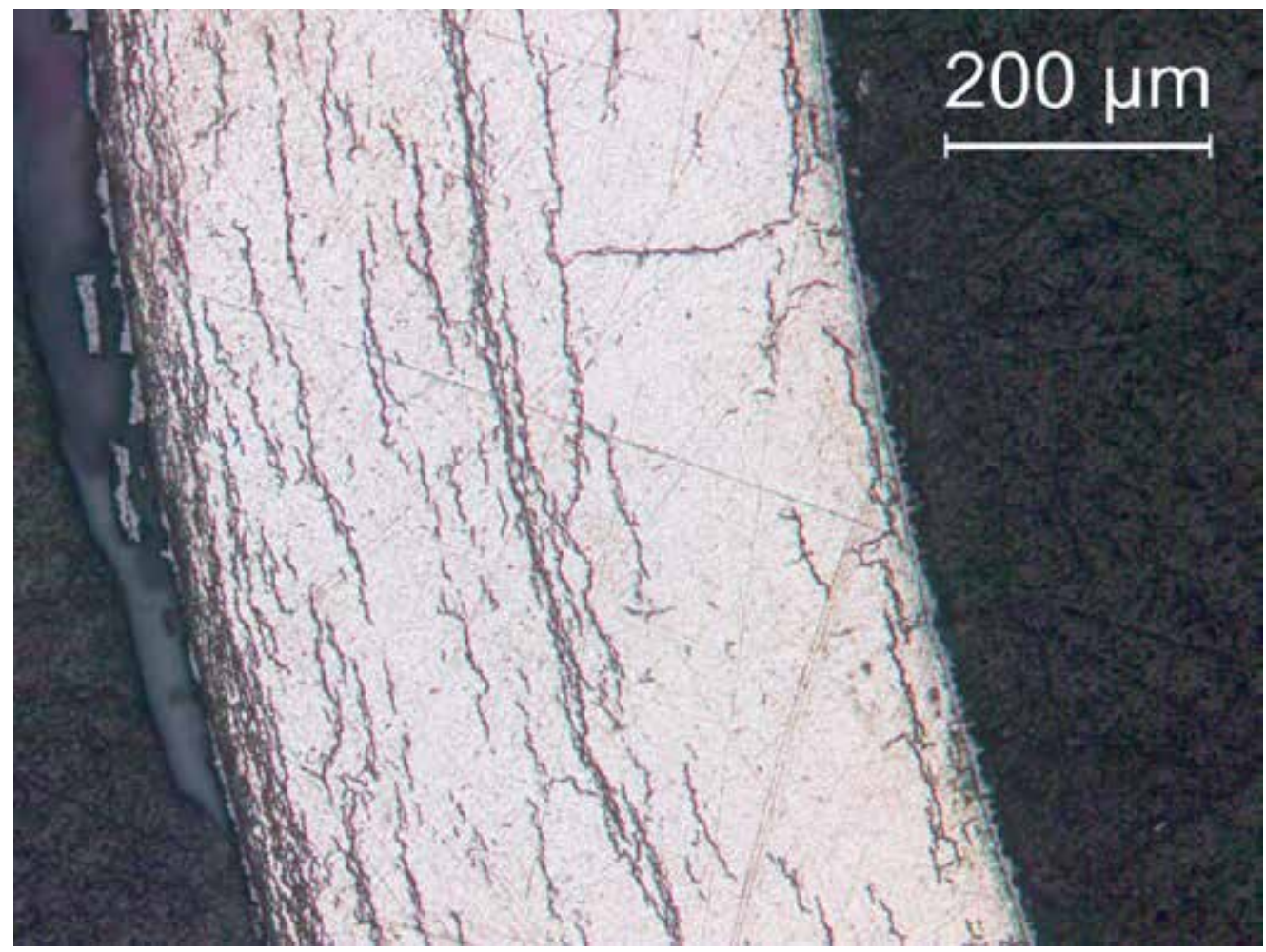

(a) $100 \mathrm{X}$

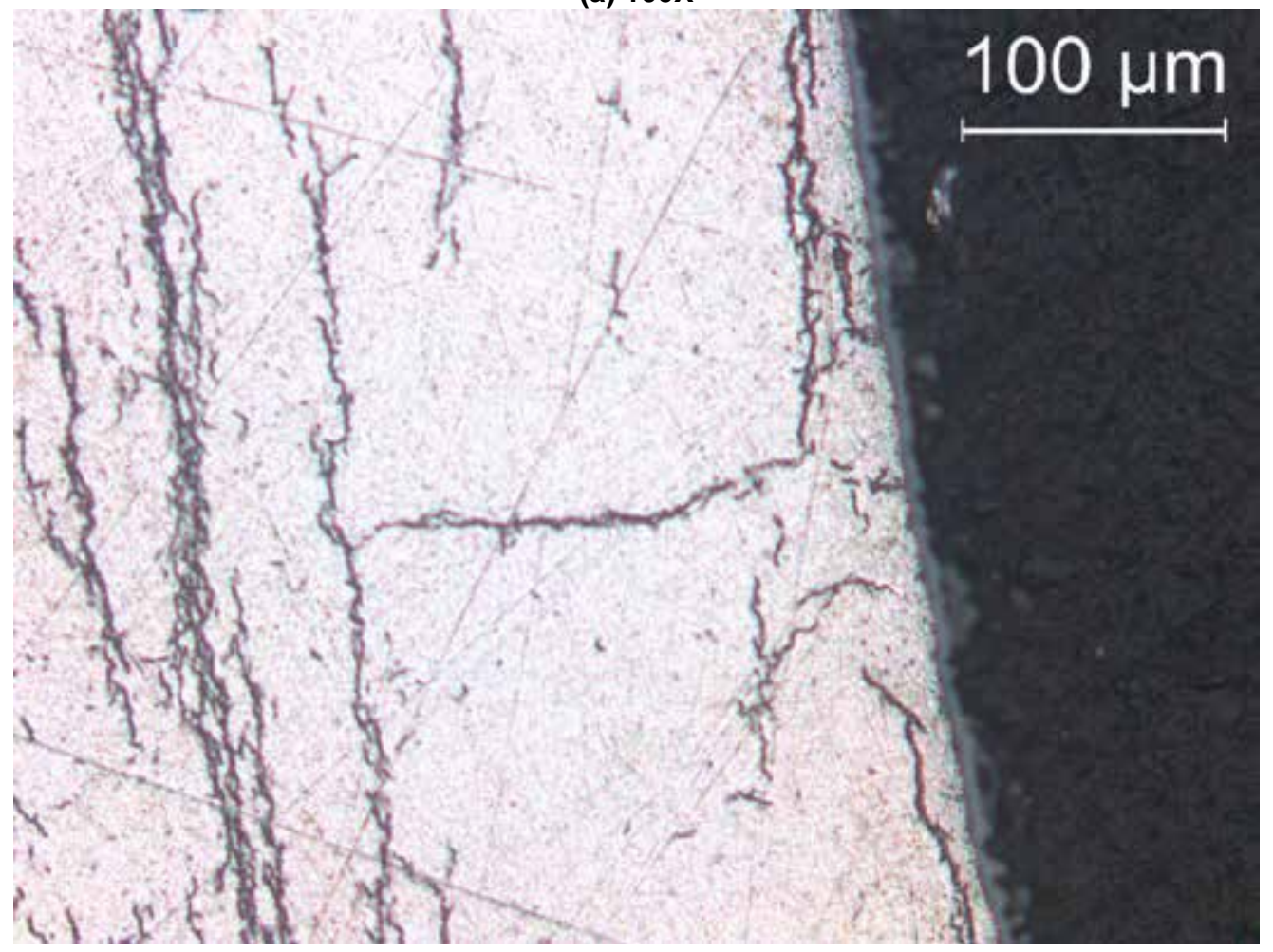

(b) $200 \mathrm{X}$

Figure 24: Images of the longest radial hydride for the 646D7A cross section $(3 \mathrm{~mm})$, which occurred at the 8:45 o'clock orientation: (a) 100X and (B) 200X. 


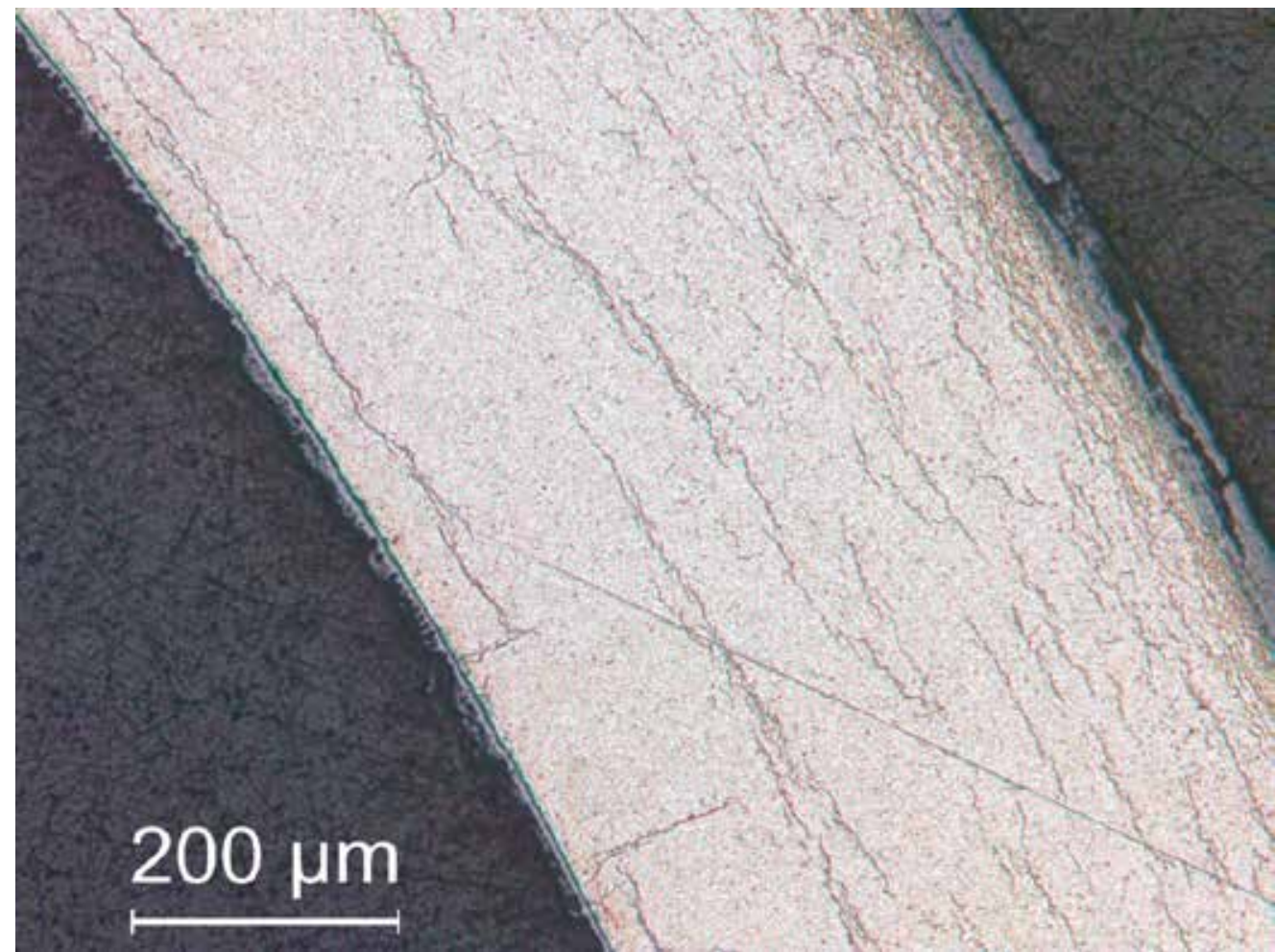

(a) $100 \mathrm{X}$

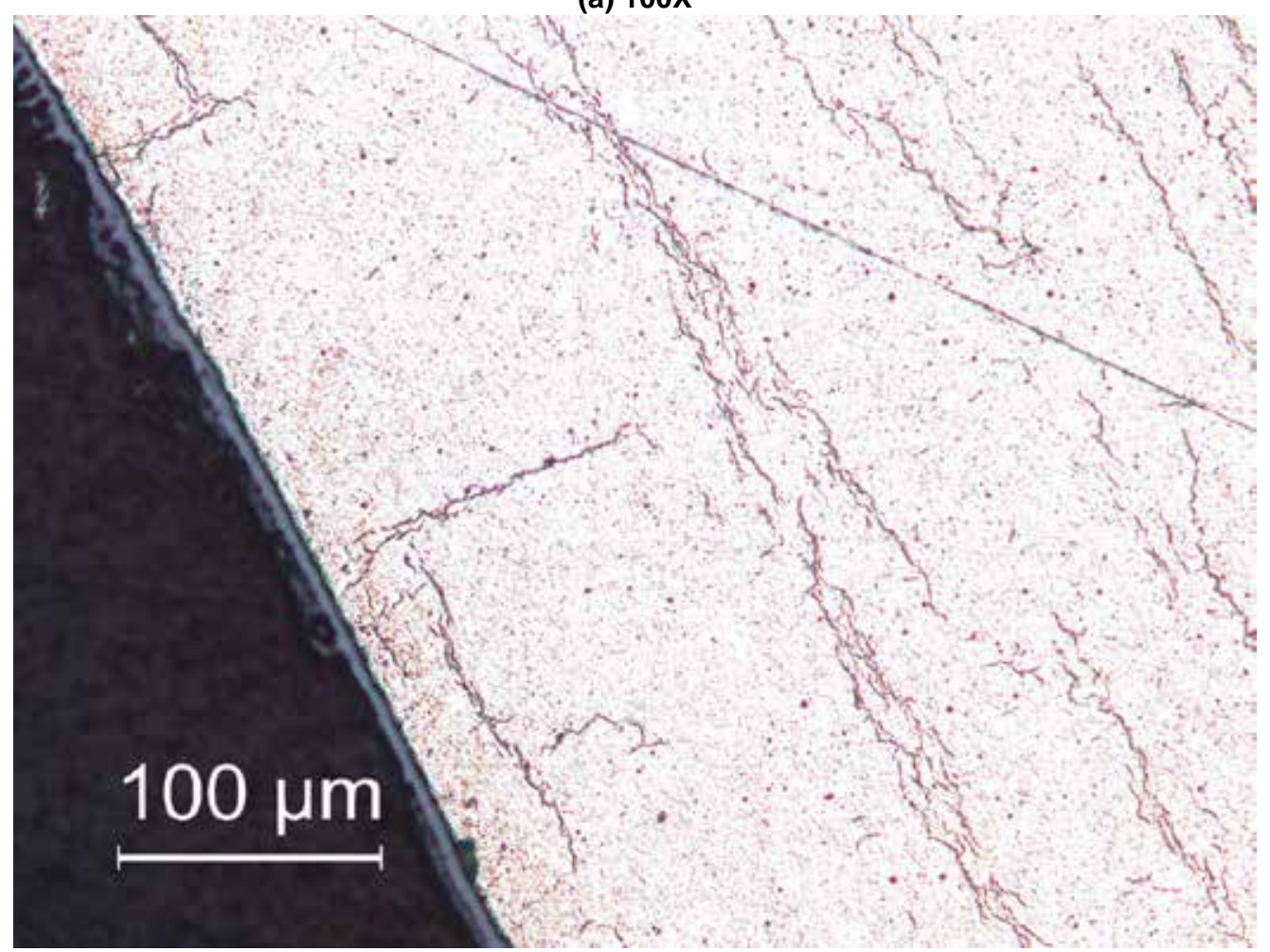

(b) $200 \mathrm{X}$

Figure 25: Images of the longest radial hydride for the 646D7B cross section (7 mm), which occurred at the 2:00 o'clock orientation: (a) 100X and (B) 200X. 
Table 4 summarizes the results of the metallographic examinations for cross sections from rodlet 646D. Averaging all RHCF values gives $15 \pm 9 \%$, which indicates relatively short radial hydrides. Although radial hydrides appear to be discontinuous in the axial direction, cracking was governed by circumferential hydrides, particularly in the dense hydride rim, which are relatively continuous in the axial direction.

Table 4 Results of metallographic examination for five cross sections of HBU ZIRLO ${ }^{\mathrm{TM}}$ rodlet 646D.

\begin{tabular}{c|c|c|c|c}
$\begin{array}{c}\text { Sample } \\
\text { ID }\end{array}$ & $\begin{array}{c}\text { Axial } \\
\text { Location, } m m\end{array}$ & $\begin{array}{c}\text { Average } \\
\text { RHCF, \% }\end{array}$ & $\begin{array}{c}\text { Peak RHCF, } \\
\%\end{array}$ & $\begin{array}{c}\text { Circumferential } \\
\text { Location of } \\
\text { Peak RHCF }\end{array}$ \\
\hline 646D4A & -6 & $17 \pm 11$ & 50 & $2: 45$ \\
646D4B & -2 & $14 \pm 6$ & 30 & $8: 45$ \\
646D6 & 0 & $20 \pm 11$ & 52 & $12: 45$ \\
646D7A & 3 & $15 \pm 8$ & 35 & $8: 45$ \\
646D7B & 7 & $10 \pm 6$ & 26 & $2: 00$ \\
\hline
\end{tabular}

\subsection{RESULTS FOR NEW HBU ZIRLO ${ }^{\text {TM }}$ RODLET 646C}

The HBU ZIRLO ${ }^{\mathrm{TM}}$ segment $646 \mathrm{C}$ was located just below the 646D segment along the parent fuel rod. Expectations were that the cladding outer diameter, oxide-metal thickness values, and hydrogen content would be about the same as for segment 646D. The pressurized and sealed $646 \mathrm{C}$ rodlet was subjected to the same thermal history as $646 \mathrm{D}$ (see Fig. 26): 24 hours at $350^{\circ} \mathrm{C}$, cooling at $4.9^{\circ} \mathrm{C} / \mathrm{h}$ to $130^{\circ} \mathrm{C}$, and exponential-decay cooling to RT. The only difference was that rodlet $646 \mathrm{C}$ was pressurized to $6.1 \mathrm{MPa}$ at $23^{\circ} \mathrm{C}$ as compared to $5.6 \mathrm{MPa}$ at $23^{\circ} \mathrm{C}$ for $646 \mathrm{D}$. The $646 \mathrm{C}$ pressure was selected to give a peak hoop stress of $93-94 \mathrm{MPa}$ at $350^{\circ} \mathrm{C}$ as compared to $87 \mathrm{MPa}$ for $646 \mathrm{D}$. Expectations were that the RHCF for $646 \mathrm{C}$ would be $>30 \%$ with a maximum value $>50 \%$ and a DTT $>120^{\circ} \mathrm{C}$.

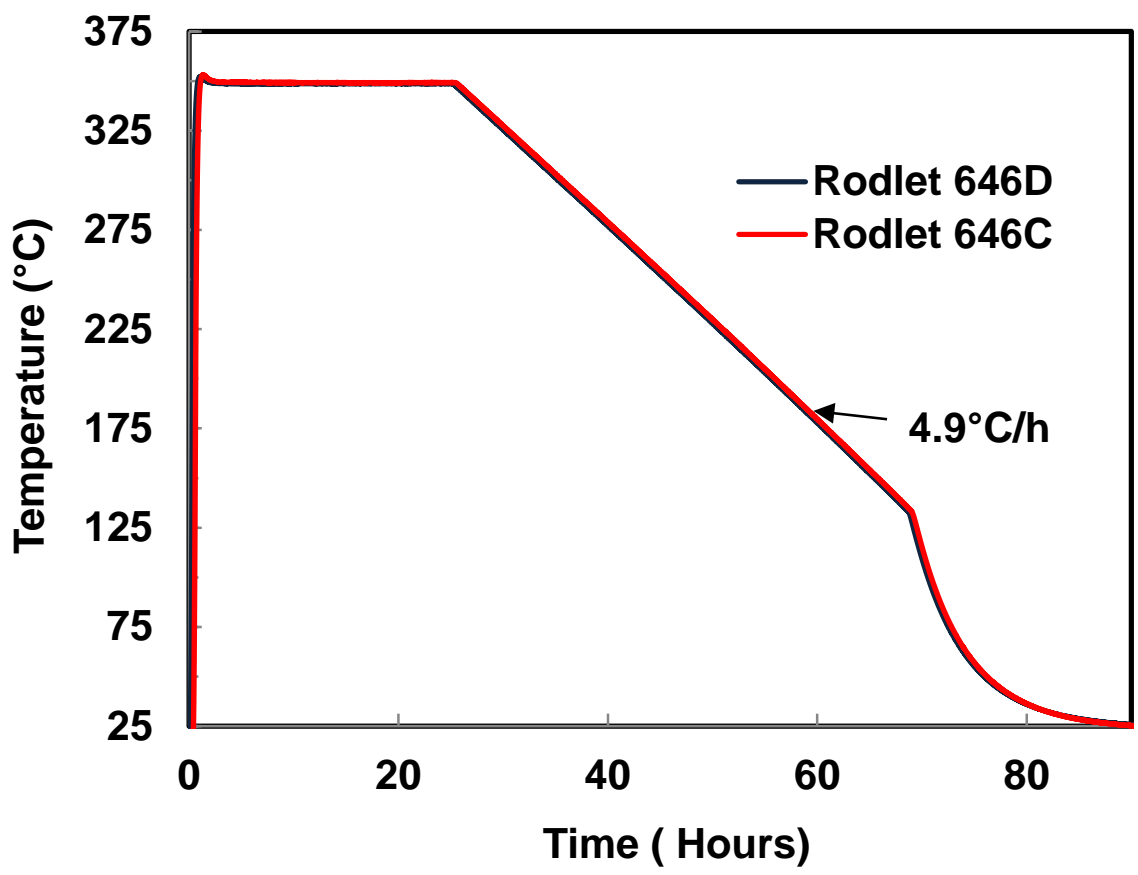

Figure 26: RHT thermal histories for rodlets $646 \mathrm{C}$ and 646D. 
Although numerous problems were encountered in fabricating rodlet $646 \mathrm{C}$, especially in performing the circumferential weld for the bottom end fixture, the pressurized and sealed sample did hold pressure in the pressurization chamber and it did pass the semi-quantitative "shake test" test both before and after RHT. Passing the shake test means that the internal zirconia pellet remained stationary during shaking. Recent tests with non-irradiated ZIRLO ${ }^{\mathrm{TM}}$ rodlets demonstrated that passing the shake test means that the internal pressure was $>3.6 \mathrm{MPa}$.

The pre-RHT outer diameter of $646 \mathrm{C}$ was measured to be $9.50 \pm 0.01 \mathrm{~mm}$, which was very close to the $646 \mathrm{D}$ pre-RHT outer diameter $(9.49 \pm 0.01 \mathrm{~mm})$. These diameters were also measured post-RHT. The post-RHT sectioning diagram for rodlet 646C is shown in Fig. 27.

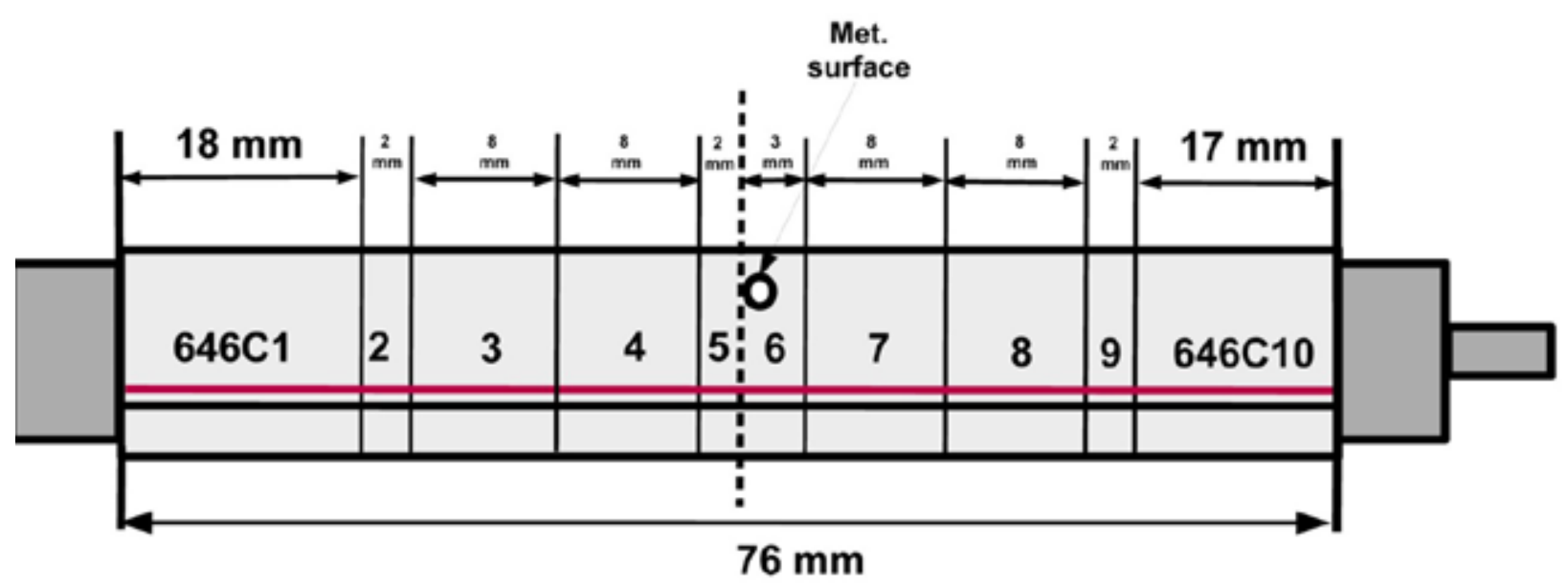

Figure 27: Sectioning diagram for HBU ZIRLO $^{\text {TM }}$ rodlet $646 \mathrm{C}$.

Metallographic examination was performed for surface $646 \mathrm{C6}$ in the as-polished condition. Oxide-layer thickness values $(30 \pm 1 \mu \mathrm{m})$ and cladding metal-wall thickness values $(0.56 \mathrm{~mm})$ were the same for rodlets $646 \mathrm{C}$ and $646 \mathrm{D}$. The ratio $\left(\mathrm{R}_{\mathrm{mi}} / \mathrm{h}_{\mathrm{m}}\right)$ in Eq. 1 for conversion of pressure difference across the cladding wall to hoop stress was slightly lower for rodlet $646 \mathrm{C}(7.40)$ than for $646 \mathrm{D}$ (7.46). However, this was accounted for in choosing the RT pressure. For the $646 \mathrm{C}$ fill pressure of $6.1 \mathrm{M} \mathrm{Pa}$, the average hoop stress across the cladding wall was calculated by Eq. 1 to be $93.6 \mathrm{M} \mathrm{Pa}$, which rounds off to $94 \mathrm{M} \mathrm{Pa}$. Thus there was excellent agreement between the target hoop stress and the hoop stress calculated from measured dimensions. Hydrogen content is yet to be determined for rings 2, 5, and 9 because of problems with the old LECO RH-404 hydrogen determinator. In the middle of diagnostic tests to assess the problems and repair the LECO machine, some of which required checking of voltages and currents, ANL issued on July 25, 2017 a stop-work order for all electrical work because of recent accidents and incidents associated with electrical work. Restart of electrical work required re-certification (in progress) of qualified electrical workers, an ANL restart plan, and restart plans for each division. It is anticipated that maintenance activities for the LECO machine will resume in early October 2017. If these activities are unsuccessful, then the new LECO ONH836 machine, which has been benchmarked and tested, will be installed in a clean glove box in Radiological Laboratory DL-114. The ONH designation indicates that this machine is capable of measuring oxygen, nitrogen and hydrogen content in metals. However, on the basis of the equivalent oxide layer thickness values, the hydrogen content in rodlet $646 \mathrm{C}$ is expected to be about the same as the hydrogen content in rodlet 646D. 
Following metallographic examination of the as-polished $646 \mathrm{C} 6$ surface, the surface was etched to allow imaging of the hydrides. Images were taken at 40 over-lapping circumferential locations. The results were quite unexpected as no radial hydrides were present on the $646 \mathrm{C} 6$ surface (see Fig. 28 at the 12:00 o'clock orientation).

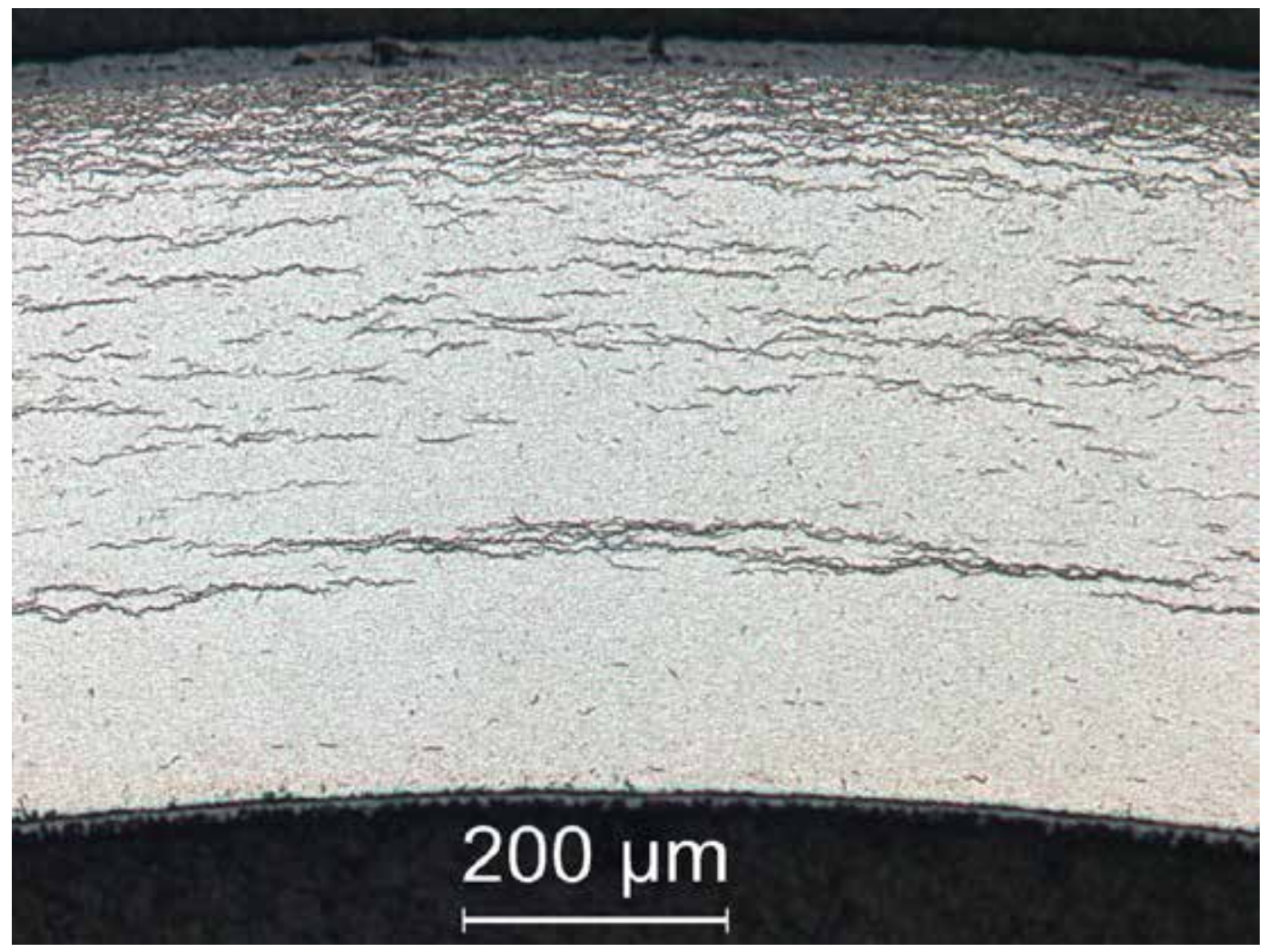

Figure 28: Micrograph from a 100X image taken at the 12:00 o'clock orientation of the $646 \mathrm{C} 6$ cross section.

Although the post-RHT rodlet passed the shake test indicating the internal pressure at RT was $>3.6 \mathrm{MPa}$, it is clear that the pressure during cooling from the precipitation temperature $\left(285^{\circ} \mathrm{C}\right)$ was lower than the pressure based on 6.1 M Pa at RT. In previous work, it had been shown for the ZIRLO ${ }^{\mathrm{TM}}$ rodlet with the lowest RT fill pressure $(4.65 \mathrm{MPa})$ that the zirconia pellet was stationary within the rodlet during the shake test and short radial hydrides ( $9 \%$ RHCF) had precipitated. Thus, the $646 \mathrm{C}$ rodlet pressure was $>3.6 \mathrm{M} \mathrm{Pa}$ and $<4.65 \mathrm{M} \mathrm{Pa}$. Extrapolating these values to $350^{\circ} \mathrm{C}$ in Eq. 1 gives bounding hoop stresses of $>55 \mathrm{M} \mathrm{Pa}$ and $<71 \mathrm{M} \mathrm{Pa}$, which is likely a relevant stress range for standard PWR fuel rods.

Because the $646 \mathrm{C}$ rodlet test did not achieve its objectives, it will be repeated using the adjacent segment $646 \mathrm{~B}$, which was just below segment $646 \mathrm{C}$ in the parent fuel rod. However, before repeating this test, it is important to understand why the test was unsuccessful. As mentioned earlier, there were problems in welding the bottom end fixture. After several unsuccessful attempts, which yielded pores and holes in the weld, the sample was inverted and the top end fixture was welded successfully after one attempt. The sample was inverted, ZIRLO chips were added to the pore-hole regions on the bottomend-fixture weld, and numerous attempts (over 20) were made to improve the weld. Following what appeared to be a sealed weld, extra weld material was ground off so that the rodlet would fit into the pressurization chamber. It is quite likely that this weld had a very small defect. However, even a small 
pin-hole flaw or a very fine through-wall crack would have resulted in complete depressurization of the rodlet, especially at elevated temperature and pressure during RHT.

Because of the extensive effort and high worker dose accumulated during fabrication of rodlet $646 \mathrm{C}$, the decision was made to proceed with the RCTs. Such tests would reveal the effects of the relatively short hydrides in the $646 \mathrm{D}$ rings as compared to the $646 \mathrm{C}$ rings with no radial hydrides. The RCTs were conducted at the same displacement rate $(5 \mathrm{~mm} / \mathrm{s})$, maximum ring displacement $(1.7 \mathrm{~mm})$ and temperatures $\left(\mathrm{RT}, 60^{\circ} \mathrm{C}, 90^{\circ} \mathrm{C}\right.$, and $120^{\circ} \mathrm{C}$ ) used to conduct RCTs for the $646 \mathrm{D}$ rings. RCT loaddisplacement curves for $646 \mathrm{C}$ rings are shown in Figs. 29-32. For ring $646 \mathrm{C} 3$ tested at $24^{\circ} \mathrm{C}$ (see Fig. 29), the $22 \%$ load drop at $5.1 \%$ offset strain was not quite enough to cause a crack $>50 \%$ of the wall. This represents the lower-bound on cladding ductility. At $11.0 \%$ offset strain, there was a significant load drop $>25 \%$, which would result in one or more long cracks, especially combined with the first load drop. However, this offset strain represents an upper bound because the actual unloading slope would be smaller than the dashed red line due to cracking that occurred during the $22 \%$ load drop. Figure 29 shows the best-estimate offset strain of $8.4 \%$. $P_{\max }$ for this $8.09-\mathrm{mm}$-long sample was $616 \mathrm{~N}$.

Figure 30 shows the RCT results for ring $646 \mathrm{C} 4$ tested at $60^{\circ} \mathrm{C}$. A $20 \%$ load drop, which was not sufficient to cause a crack extending through $>50 \%$ of the cladding wall, occurred at $6.5 \%$ offset strain, which represents a reasonable lower bound on cladding ductility. The ductility would be slightly higher if the sample were unloaded at the full sample displacement of $1.7 \mathrm{~mm}$. However, the unloading slope would be significantly lower than $0.68 \mathrm{kN} / \mathrm{mm}$ due to cracking. If post-RCT metallographic examination reveals that cracks are $<50 \%$ of the wall thickness, then a more accurate determination of the ductility would be the permanent strain (8.9\%). $P_{\max }$ for this 7.98-mm-long sample was $594 \mathrm{~N}$.

Figure 31 shows the RCT results for ring $646 \mathrm{C} 8$ tested at $90^{\circ} \mathrm{C}$. There is a gradual load drop of $8 \%$ between the displacement at maximum load and the end of the 1.7-mm sample displacement. On the basis of previous experience, this gradual and mild load drop is associated with short cracks that develop within the hydride rim, especially around the 3:00 and 9:00 o'clock orientations for which the maximum tensile stress occurs at the cladding outer surface. The offset and permanent strains were determined to be $10.3 \%$ and $9.3 \%$, respectively. For this case, the permanent strain is a more accurate representation of ductility. $P_{\max }$ for this 7.65-mm-long sample was $549 \mathrm{~N}$. Similar results are shown in Fig. 32 for ring $646 \mathrm{C} 7$ tested at $120^{\circ} \mathrm{C}$. Offset and permanent strains were determined to be $11.1 \%$ and $10.5 \%$, respectively. Although the permanent strain is more accurate, both round off to $11 \%$ giving a ductility $>11 \%$. $P_{\max }$ for this $8.06-\mathrm{mm}$-long sample was $563 \mathrm{~N}$.

Table 5 shows the comparison between RCT results for $646 \mathrm{C}$ rings with no radial hydrides and 646D rings with relatively short radial hydrides $(15 \pm 9 \%)$. Results for ductility and maximum load (normalized to a length of $8 \mathrm{~mm}$ ) are comparable at test temperatures of $60^{\circ} \mathrm{C}, 90^{\circ} \mathrm{C}$, and $120^{\circ} \mathrm{C}$. However, the $646 \mathrm{D}$ ring tested at RT had considerably lower ductility (1.9\% vs. $8.4 \%)$, more significant cracking as indicated by the multiple load drops, and lower (16\%) maximum load due to the cracking. Thus the contribution of short radial hydrides to embrittlement was only observed at RT. 


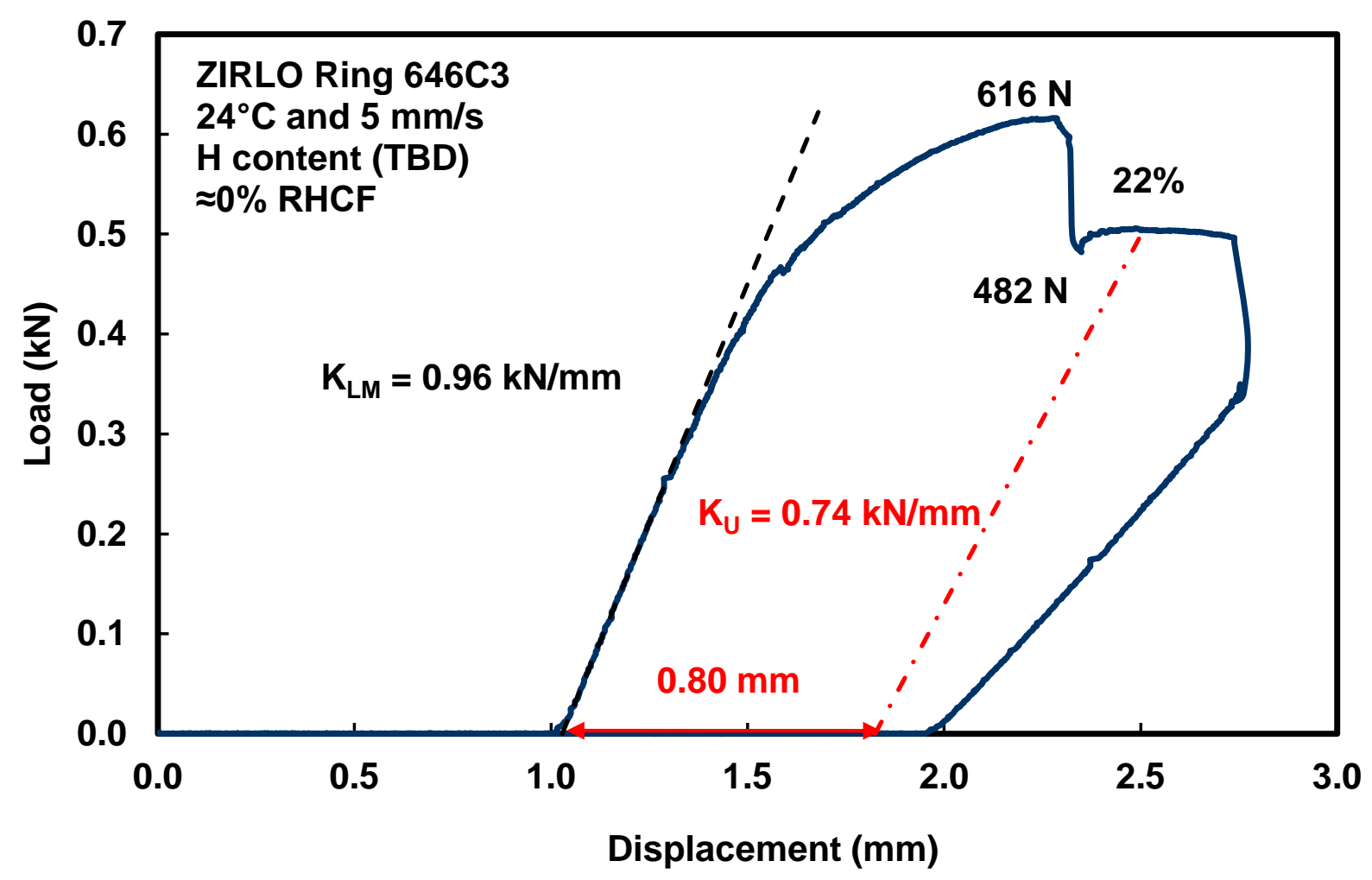

Figure 29: Load-displacement curve for the $646 \mathrm{C} 3$ sample tested at $24^{\circ} \mathrm{C}$.

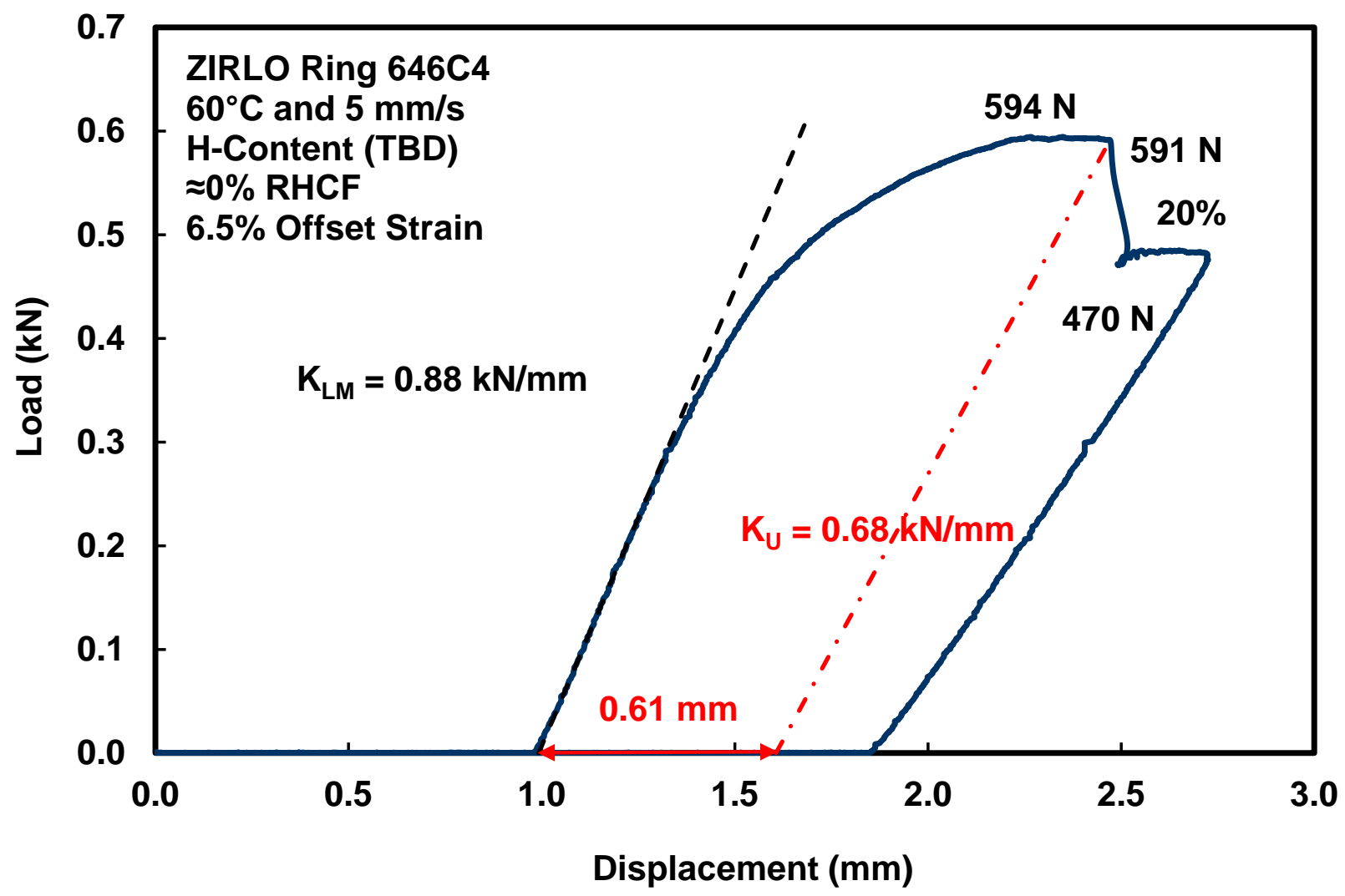

Figure 30: Load-displacement curve for the $646 \mathrm{C} 4$ sample tested at $60^{\circ} \mathrm{C}$. 


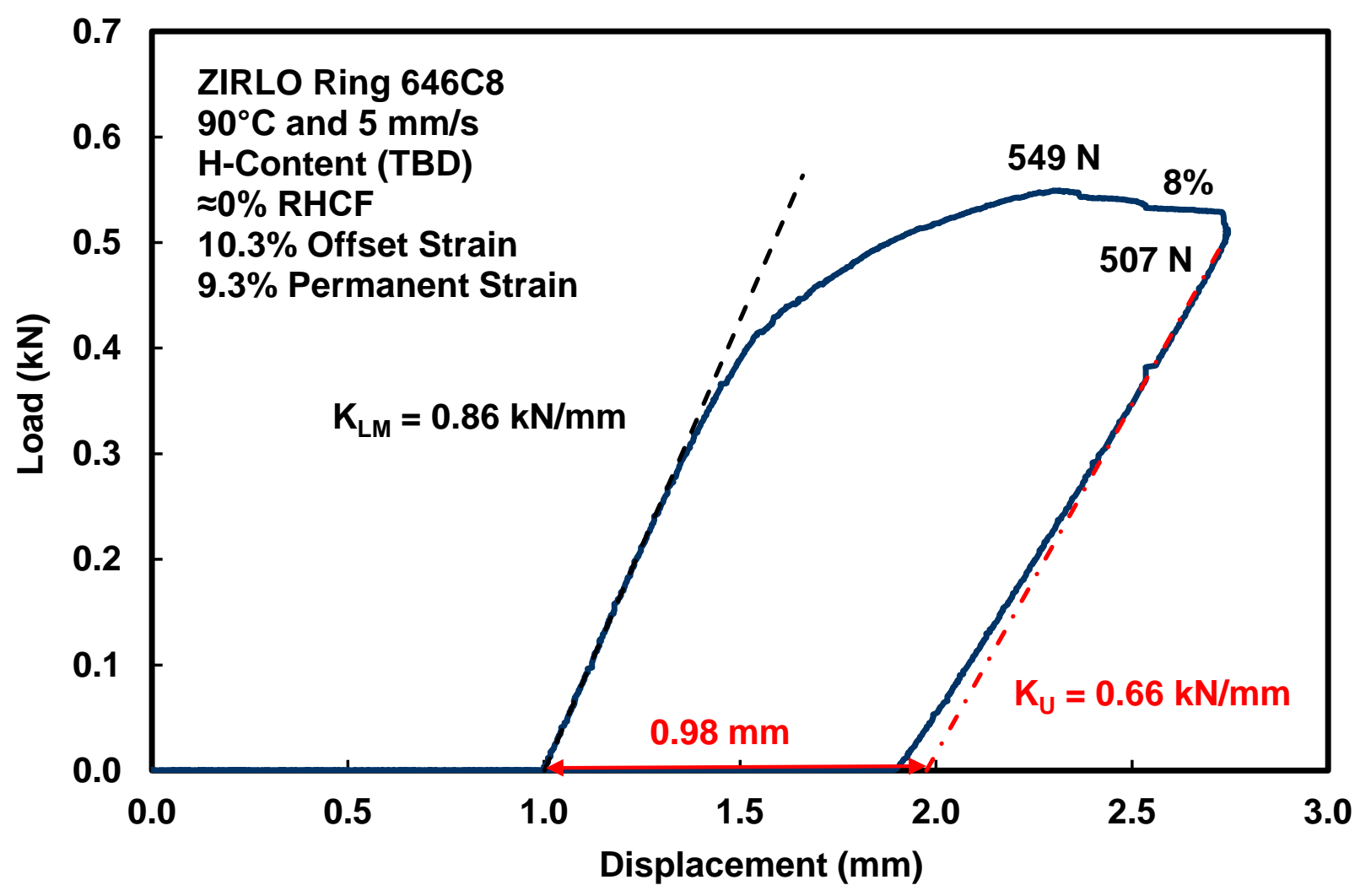

Figure 31: Load-displacement curve for the $646 \mathrm{C} 8$ sample tested at $90^{\circ} \mathrm{C}$.

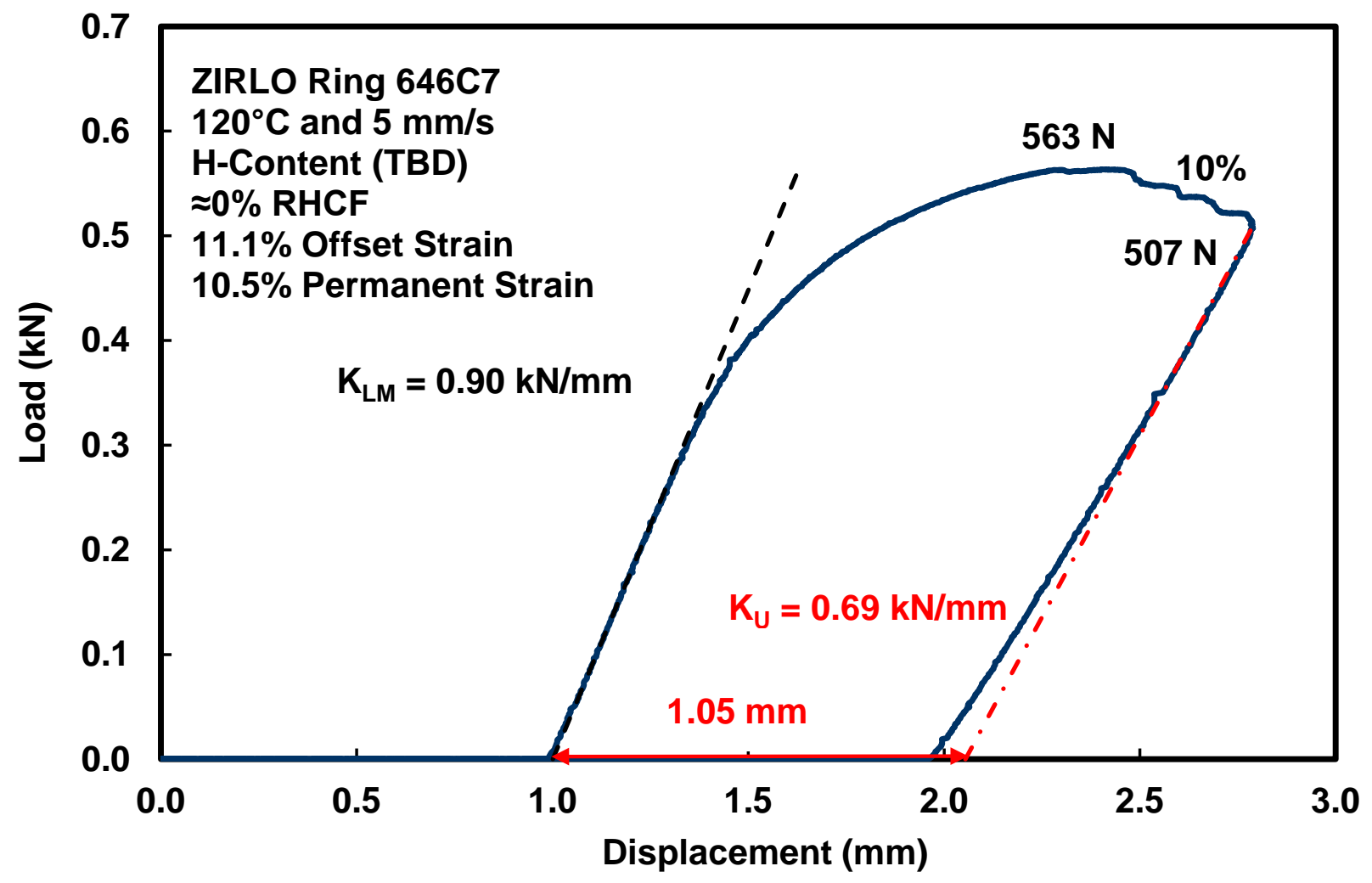

Figure 32: Load-displacement curve for the $646 \mathrm{C} 7$ sample tested at $120^{\circ} \mathrm{C}$. 
Table 5 RCT results for HBU ZIRLO ${ }^{\text {TM }}$ rodlets 646C (0\% RHCF) and 646D (15 $\pm 9 \%$ RHCF).

\begin{tabular}{c|c|c|c|c|}
$\begin{array}{c}\text { Sample } \\
\text { ID }\end{array}$ & $\begin{array}{c}\text { RCT } \\
\text { Temperature, } \\
{ }^{\circ} \mathrm{C}\end{array}$ & $\begin{array}{c}\text { Offset } \\
\text { Strain, \% }\end{array}$ & $\begin{array}{c}\text { Permanent } \\
\text { Strain, } \\
\%\end{array}$ & $\begin{array}{c}\text { (8.0-mm/L) } \text { Pmax }_{\text {max }} \\
\text { N }\end{array}$ \\
\hline $646 \mathrm{C3}$ & 24 & 8.4 & - & 609 \\
$646 \mathrm{D} 8$ & 28 & 1.9 & - & 514 \\
& & & & \\
$646 \mathrm{C} 4$ & 60 & 6.5 & 8.9 & 595 \\
$646 \mathrm{D} 4$ & 60 & 8.0 & - & 577 \\
& & & & \\
$646 \mathrm{C} 8$ & 90 & $>10.3$ & $>9.3$ & 549 \\
$646 \mathrm{D} 7$ & 90 & $>9.9$ & $>8.4$ & 542 \\
& & & & \\
$646 \mathrm{C} 7$ & 120 & $>11.1$ & $>10.5$ & 558 \\
$646 \mathrm{D} 3$ & 120 & $>9.6$ & $>8.9$ & 575 \\
\hline
\end{tabular}




\section{DISCUSSION AND SUMMARY}

\section{Significance of the Maximum Load ( $\left.\mathbf{P}_{\max }\right)$ Achieved during RCTs}

The RCT has been used in ANL work as a ductility screening test. The focus was on determining cladding ductility as a function of test temperature and the resulting ductility transition temperature (DTT). Sensitivity of the DTT was investigated as a function of peak RHT temperature and hoop stress, hoop stress at the time and temperature that new hydrides would precipitate, texture for RXA and CWSRA cladding alloys, hydrogen content and distribution of circumferential hydrides across the cladding wall. $P_{\max }$ was recorded for benchmark tests with as-fabricated cladding and for tests with irradiated cladding rings with and without RHT, but it was not used to assess the strength of the cladding. As shown in Section 2.2, $P_{\max }$ is proportional to $L\left(h_{m} / R_{\operatorname{mid}}\right)^{3}$ in the elastic deformation regime. It also has a strong linear dependence on the elastic displacement. However, the increase of $P_{\max }$ with increasing displacement in the elastic-plastic regime is very gradual in the displacement range from $1.0 \mathrm{~mm}$ to 1.7 $\mathrm{mm}$ for as-fabricated $M 5 \otimes P_{\max }$ results for $M 5 \AA$ samples with wall thickness values of $0.61 \mathrm{~mm}$ (reference case) and $0.57 \mathrm{~mm}$ agreed quite well when normalized by $L\left(\mathrm{~h}_{\mathrm{m}} / \mathrm{R}_{\mathrm{mid}}\right)^{2.44}$. This relationship was found to be independent of test temperature and displacement rate. Although more results for 0.57$\mathrm{mm}$ wall samples are needed, $\mathrm{P}_{\max }$ can now be used to estimate the degree of irradiation hardening in M 5 @for HBU M 5 ®cladding rings that exhibited no evidence of cracking during ring-compression testing. For test conditions of $25^{\circ} \mathrm{C}$ and $0.05 \mathrm{~m} / \mathrm{s}$, the increase in the normalized $P_{\max }$ for $\mathrm{HBU} \mathrm{M} 5 \otimes$ was $46 \%$ as compared to as-fabricated $\mathrm{M} 5{ }^{\circledR}$ At $25^{\circ} \mathrm{C}$ and $5 \mathrm{~mm} / \mathrm{s}$ (reference displacement rate), the increase was $40 \%$. At $120^{\circ} \mathrm{C}$ and $5 \mathrm{~mm} / \mathrm{s}$, the increase was $35 \%$. Correlations for hoop tensile properties for asfabricated and HBU M 5 ®are given in Ref. 17 based on very high-strain-rate $(500 \% / \mathrm{s})$ ring stretch tests. At $25^{\circ} \mathrm{C}$, the yield strength of $\mathrm{HBU} \mathrm{M} 5{ }^{\circledR i n c r e a s e d}$ by $50 \%$ and the ultimate tensile strength increased by $25 \%$. These values are consistent with the increases seen in $P_{\max }$. It should be noted that $P_{\max }$ can, in principle, be used to assess the degree of irradiation-damage annealing in HBU M $5 \AA$.

\section{Effects of Irradiation-Damage Annealing}

The most common method used to assess recovery of irradiation damage or hardening is the hardness test [18]. ASTM standards are available for various hardness tests (e.g., see ASTM E 92 - 82 for Vickers Hardness Tests of Metallic M aterials). It is also common to perform such measurements following longtime (up to a year) creep tests. Other methods used are biaxial tube tests and ring-stretch tensile tests [19]. Although the RCT is not proposed as a replacement for these test methods, $P_{\max }$ results can be used to assess the degree of annealing that may have occurred in HBU M 5®during RHT. For Zry-4 and ZIRLO ${ }^{\text {TM }}$ cladding alloys with hydride rims, "softening" of the ring due to partial-wall cracks initiating at the oxiderim boundary is likely to be greater than softening due to annealing. Even if the two mechanisms had comparable effects on reducing $P_{\max }$, softening due to cracking would obscure the effects of softening due to annealing.

The short-time (up to 24 hours at the PCT) ANL tests are not designed to study annealing. Although partial annealing would improve ductility and lower the DTT, we believe that it can be demonstrated that cladding hoop stresses are too low for PWR fuel rods to induce embrittlement in HBU cladding with full irradiation hardening.

It is important to address the issue of annealing in the sister-rod test program because of the long times that the cladding will be at elevated temperature. Also, the annealing rate needs to be established as a function of cold work (i.e., texture) and hydrogen content, as RXA alloys such as M 5 ®with low hydrogen 
content are expected to anneal much faster than cold-worked alloys with high hydrogen content. Although such annealing would reduce the likelihood of radial-hydride-induced embrittlement, it would also reduce the strength properties of the cladding. Cask vendors currently use the strength properties (see Ref. 1) of as-irradiated cladding with full hardening in their structural analyses. If annealing during storage proves to be significant, then this practice is non-conservative.

\section{Axial Continuity of Circumferential Hydrides, Radial Hydrides, and Post-RCT Cracks}

Previous results for HBU M 5®indicated a critical hydrogen content below which radial-hydride-induced embrittlement did not occur at relevant storage and transportation temperatures. Lower hydrogen contents result in lower precipitation temperatures and lower precipitation stresses for the same peak $\mathrm{RHT}$ temperatures and stresses. Also, it is reasonable to assume that lower hydrogen contents result in more axial discontinuity of radial hydrides even if all the hydrogen precipitates as radial hydrides. In an effort to better quantify the degree of axial continuity or connectivity of radial hydrides in $M 5 \AA$, six cross sections from rodlet $652 \mathrm{~F} 2\left(350^{\circ} \mathrm{C} / 89-\mathrm{MPa}\right.$ peak RHT conditions) with 80 wppm hydrogen were examined metallographically: (a) three cross sections within $0.6 \mathrm{~mm}$ of a ring not subjected to the RCT and (b) three cross sections from two post-RCT rings. On the basis of average and peak RHCF and circumferential location of the peak RHCF, it was clearly shown that the radial hydrides were not continuous. However, for the brittle sample tested at RT, the two major cracks that developed appeared to be continuous based on metallographic examination of the mid-span and visual inspection of the two ends of the ring. This result suggests that cracks can propagate along the axial direction even if there was axial discontinuity of radial hydrides in $\mathrm{HBU} \mathrm{M}^{\circledR}{ }^{\circledR}$ samples with $\geq 80$ wppm hydrogen.

Five cross sections of $\mathrm{HBU} Z \mathrm{ZRLO}{ }^{\mathrm{TM}}$ rodlet $646 \mathrm{D}\left(350^{\circ} \mathrm{C} / 87-\mathrm{MPa}\right.$ peak RHT conditions) were examined metallographically: (a) one from a short ring that had not been subjected to the RCT and (b) four from two post-RCT rings. The material contained about $390 \mathrm{wppm}$ hydrogen, exhibited a well-developed hydride rim and contained circumferential hydrides that appeared to have a high degree of continuity in the axial direction. Radial hydrides were relatively short (15 $\pm 9 \%$ of the cladding wall) and likely contained lower hydrogen content than the M5 ${ }^{\circledR}$ rodlet 652F2. Average and peak RHCF values varied from $10 \%$ to $20 \%$ and from $26 \%$ to $52 \%$, respectively, and the circumferential locations of the peak values varied considerably. However, for the sample compressed at $60^{\circ} \mathrm{C}$, a long wall crack was present at the 3 o'clock orientation of the mid-span and end cross-sections. The crack, which initiated in the hydride rim, appeared to have a high degree of axial continuity due to the axial continuity of circumferential hydrides.

\section{Effects of Cladding Hoop Stress on Radial Hydride Precipitation and the DTT}

For HBU M $5 \circledR$ with 72-94 wppm hydrogen, the DTT exhibited very little stress sensitivity following RHT at $350^{\circ} \mathrm{C}$ and $400^{\circ} \mathrm{C} \mathrm{PCT}, 89-142 \mathrm{MPa}$ peak hoop stress, and 74-113 MPa precipitation hoop stress. The $\mathrm{DTT}$ was in the range of $75 \pm 5^{\circ} \mathrm{C}$ for these cases. Radial hydrides were longest for the $94-\mathrm{wppm} / 142-\mathrm{M} \mathrm{Pa}$ rodlet and, on average, did decrease with decreasing hydrogen content and hoop stress.

On the basis of previous test results for HBU ZIRLO ${ }^{\mathrm{TM}}$ rodlets, the DTT for this material exhibited a very high sensitivity to peak and precipitation-temperature hoop stresses. For the narrow peak stress range of $90 \pm 3 \mathrm{MPa}$, the DTT increased by almost $100^{\circ} \mathrm{C}$. However, the higher-stress case (93 M Pa) also contained higher hydrogen content and a thicker hydride rim than the lower stress cases (87-89 MPa). A new HBU ZIRLO ${ }^{\mathrm{TM}}$ rodlet (646C) with an estimated 390 wppm hydrogen was fabricated with higher RT pressure calculated to give a peak hoop stress of about $93 \mathrm{MPa}$ at $350^{\circ} \mathrm{C}$. It had the same oxide-layer thickness and metal-wall thickness as rodlet 646D (390 wppm hydrogen) and was subjected to the same 
RHT thermal history as rodlet 646D. However, it was quite evident that this rodlet did not attain the designated pressure necessary to achieve $93 \mathrm{MPa}$ at $350^{\circ} \mathrm{C}$. This was clear by the absence of radial hydrides in the polished-and-etched surface imaged at 100X and 200X. The peak hoop stress was shown to be in the range of $63 \pm 8 \mathrm{MPa}$. Although this test did not achieve its objectives, it is shown in the next section that $63 \pm 8 \mathrm{MPa}$ is a relevant peak hoop stress for PWR ZIRLO ${ }^{\mathrm{TM}}$-clad fuel rods during storage.

\section{Relevant Ranges of Internal Pressures and Hoop Stresses}

ANL and other DOE Laboratories have been working with the Electric Power Research Institute (EPRI), fuel vendors, cask vendors, and the international community to determine relevant ranges of end-of-life (EOL) PWR rod-internal pressure (RIP) values, as well as PCTs and rod-averaged gas temperatures. EPRI documented results for publicly available PWR EOL RIP data at $25^{\circ} \mathrm{C}$ [20]. Characterization of the sister rods will result in up to 25 valuable data points for EOL RIP for standard PWR fuel rods. In addition, Oak Ridge National Laboratory used FRAPCON to predict gas pressures for over 68,000 fuel rods irradiated during Cycles 1-12 in the Watts Bar Nuclear Unit 1 reactor [21]. The calculations documented in Ref. 21 were consistent with the database documented in Ref. 20 for standard PWR fuel rods. Both the database and the calculations for standard PWR fuel rods irradiated to 40-60 GW d/MTU were bounded by $5 \mathrm{MPa}$ EOL RIP at $25^{\circ} \mathrm{C}$. However, the calculated EOL RIP values for ZIRLO ${ }^{\mathrm{TM}}$-clad Integral Fuel Burnable Absorber (IFBA with B-10) fuel rods appeared to be too high and no EOL RIP data were available for such rods at that time to validate models for He gas release from IFBA rods.

Quite recently, NRC sponsored the Pacific Northwest National Laboratory to perform FRAPCON calculations under drying and storage conditions for boiling water reactor, standard PWR, and IFBA PWR fuel rods using a validated model for gas release from the B-10-containing absorber on the outer surface of the fuel pellets for IFBA fuel rods [22]. For standard PWR fuel rods, the peak hoop stress occurred during storage and was bounded by $53 \mathrm{MPa}$. For PWR IFBA rods, peak hoop stresses also occurred during storage and were bounded by $81 \mathrm{M} \mathrm{Pa}$. If these calculations adequately bound PWR hoop stresses during storage, then radial hydride precipitation is not predicted to occur for standard Zry-4-clad and $Z^{Z} R_{R L O}{ }^{\mathrm{TM}}$-clad PWR fuel rods during cooling from peak drying-transfer-storage temperatures. The recently tested $\mathrm{HBU}$ ZIRLO ${ }^{\mathrm{TM}}$ rodlet $646 \mathrm{C}$ had $>55 \mathrm{M} \mathrm{Pa}$ cladding hoop stress at $350^{\circ} \mathrm{C}$ and resulted in no radialhydride precipitation during cooling. $\mathrm{HBU} M 5 \circledast$ standard fuel rods may precipitate some radial hydrides during cooling from peak storage temperatures, but the DTT for such rods should remain low. Given that PWR IFBA rods are predicted to be bounded by 81-M Pa peak stress during storage, it is worthwhile to repeat the $\mathrm{HBU} Z \mathrm{ZIRLO}{ }^{\mathrm{TM}}$ rodlet test with $\mathrm{RHT}$ peak conditions of $350^{\circ} \mathrm{C}$ and $93 \mathrm{MPa}$ to determine the stress margin for such rods to maintain a $\mathrm{DTT}<50^{\circ} \mathrm{C}$. 
Page intentionally blank 


\section{REFERENCES}

[1] Geelhood, K.J., W.G. Lusher, and C.E. Beyer, PNNL Stress/Strain Correlation for Zircaloy, Pacific Northwest National Laboratory Report PNNL-17700, July 2008.

[2] Nuclear Regulatory Commission, Interim Staff Guidance (ISG)-11, Revision 3, "Cladding Considerations for the Transportation and Storage of Spent Fuel," November 2003. M L033230335 at http://www.nrc.gov/ reading-rm/adams.html

[3] Billone, M.C., T.A. Burtseva, and Y. Yan, Ductile-to-Brittle Transition Temperature for High-Burnup Zircaloy-4 and ZIRLO ${ }^{\mathrm{TM}}$ Cladding Alloys Exposed to Simulated Drying-Storage Conditions, Argonne National Laboratory Report ANL-13/13, NRC ADAM S M L12181A238, Sept. 2012.

[4] Billone, M.C., T.A. Burtseva, and R.E. Einziger, "Ductile-to-Brittle Transition Temperature for HighBurnup Cladding Alloys Exposed to Simulated Drying-Storage Conditions," J. Nucl. Mater. 433, 431-448 (2013).

[5] Billone, M.C., T.A. Burtseva, J.P. Dobrzynski, D.P. M cGann, K. Byrne, Z. Han, and Y.Y. Liu, Phase I Ring Compression Testing of High-Burnup Cladding, FCRD-USED-2012-000039, Dec. 31, 2011.

[6] Billone, M.C., T.A. Burtseva, and Y.Y. Liu, Baseline Studies for Ring Compression Testing of HighBurnup Fuel Cladding, Argonne National Laboratory Report ANL-12/58, FCRD-USED-2013-000040, Nov. 23, 2012.

[7] Billone, M.C., T.A. Burtseva, and Y.Y. Liu, "Effects of Drying and Storage on High-Burnup Cladding Ductility," Proc. IHLRWM C, Albuquerque, NM , April 28-M ay 2, 2013, Paper 6973, 1106-1113 (2013).

[8] Billone, M.C., T.A. Burtseva, and Y.Y. Liu, "Baseline Properties and DBTT of High-Burnup PWR Cladding Alloys," Proc. PATRAM 2013, San Francisco, CA, August 18-23, 2013.

[9] Billone, M.C., T.A. Burtseva, Z. Han, and Y.Y. Liu, Embrittlement and DBTT of High-Burnup PWR Fuel Cladding Alloys, Argonne National Laboratory Report ANL-13/16, FCRD-UFD-2013-000401, Sept. 30, 2013.

[10] Billone, M.C., T.A. Burtseva, Z. Han, and Y.Y. Liu, Effects of M ultiple Drying Cycles on High-Burnup PWR Cladding Alloys, Argonne National Laboratory Report ANL-14/ 11, FCRD-UFD-2014-000052, Sept. 26, 2014.

[11] Billone, M.C., T.A. Burtseva, and M.A. M artin-Rengel, Effects of Lower Drying-Storage Temperatures on the DBTT of High-Burnup PWR Cladding, Argonne National Laboratory Report ANL-15/21, FCRD-UFD-2015-000008, Aug. 28, 2015.

[12] Billone, M.C. and T.A. Burtseva, Effects of Lower Drying-Storage Temperatures on the Ductility of High-Burnup PWR Cladding, Argonne National Laboratory Report ANL-16/16, FCRD-UFD-2016000065, Aug. 30, 2016.

[13] Kearns, J.J., "Terminal Solubility and Partitioning of Hydrogen in the Alpha Phase of Zrconium, Zircaloy-2 and Zrcaloy-4," J. Nucl. M ater. 22, 292-303, 1967. 
[14] Kammenzind, B.F., D.G. Franklin, H.R. Peters, and W.J. Duffin, “Hydrogen Pickup and Redistribution in Alpha-Annealed Zircaloy-4," Zirconium in the Nuclear Industry: $11^{\text {th }}$ Intl. Symp., ASTM STP 1295, E.R. Bradley and G.P. Sabol, Eds., ASTM , pp. 338-370, 1996.

[15] M cM inn, A., E.C. Darby, and J.S. Schofield, "The Terminal Solid Solubility of Hydrogen in Zrconium Alloys, Zirconium in the Nuclear Industry: $12^{\text {th }}$ Intl. Symp., ASTM STP 1354, G.P. Sabol and G.D. Moan, Eds., ASTM , pp. 173-195, 2000.

[16] Tang, R. and X. Yang, "Dissolution and precipitation behaviors of hydrides in N18, Zry-4 and M $5{ }^{\circledR}$ alloys," Intl. J. Hydrogen Energy, 34 (2009) 7269-7274.

[17] Fourgeaud, S., J. Desquines, M. Petit, C. Getrey and G. Sert, "M echanical characteristics of fuel rod claddings in transport conditions," Packaging, Transport, Storage \& Security of Radioactive Material, 20, W.S. Maney \& Son Ltd (2009) 69-76.

[18] Ito, K., K. Kamimura, and Y. Tsukuda, "Evaluation of Irradiation Effect on Spent Fuel Cladding Creep Properties," Proceed. 2004 Intl. Meet. On LWR Fiel Perform., Orlando, FL, Sept. 19-22, 2004, Paper 1117 (2004) 440-451.

[19] Bouffioux, P., A. Ambard, A. M iquet, C. Cappelaere, Q. Auzoux, M. Bono, O. Rabouille, S. Allegre, V. Chambretou, and C.P. Scott, "Hydride Reorientation in M $5 \circledR C l a d d i n g$ and its Impact on M echanical Properties," LWR Fuel Perf. M eet., Top Fuel 2013, 2 (2013) 879-886.

[20] Machiels, A., End-of-Life Rod Internal Pressures in Spent Pressurized Water Reactor Fuel, EPRI Report 3002001949, Dec. 2013.

[21] Bratton, R.N., M.A. Jessee, and W.A. Wieselquist, Rod Internal Pressure Quantification and Distribution Analysis Using FRAPCON, DOE Report FCRD-UFD-2015-000636, ORNL Report ORNL/TM -2015/557, Sept. 30, 2015.

[22] Richmond, D.J. and K.J. Geelhood, "FRACON Analysis of Cladding Performance during Dry Storage Operations," WRFPM 2017, Jeju Island, Korea, Sept. 10-14, 2017. 